Distribution Category:

General Reactor Technology

(UC-80)

ANL-82-72

AillL- $-82-72$

DEQ3 014262

\author{
ARGONNE NATIONAL LABORATORY \\ 9700 South Cass Avenue \\ Argonne, Illino is 60439
}

\title{
FUEL-MOTION DIAGNOSTICS \\ AND CINERADIOGRAPHY
}

by

A. DeVolpi

Reactor Analys is and Safety Division

\section{DISCLAIMER}

\begin{abstract}
This report was prepared as an account of work sponsored by an agency of the United States Government. Neither the United States Government nor any agency thereof, nor any of their employees, makes any warranty, express or implied, or assumes any legal liability or responsibility for the accuracy, completeness, or usefulness of any information, apparatus, product, or process disclosed, or represents that its use would not infringe privately owned rights. Reference herein to any specific commercial product, process, or service by trade name, trademark, manufacturer, or otherwise does not necessarily constitute or imply its endorsement, recommendation, or favoring by the United States Government or any agenc, thereor. The views and opinions of authors expressed herein do not necessarily state or reflect those of the United States Government or any agency thereof.
\end{abstract}

September 1982

\section{NOTIGE}

PORTIONS OF THIS REPOTT ARE ILLEGBLE. It has been reproduced from the best available copy to permit the broadest possible availability. 


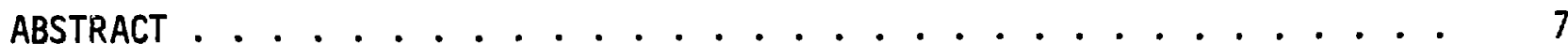

I. INTRODUCTION . . . . . . . . . . . . . . . . . . 7

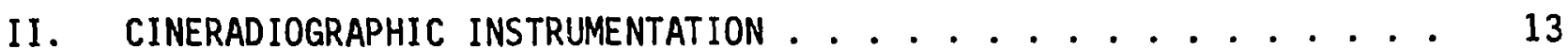

III. HODOSCOPE DIAGNOSTIC INSTRUMENTATION . . . . . . . . . . . 15

A. Description of apparatus . . . . . . . . . . . . . 16

1. TREAT hodoscope ................. 16

2. CABRI hodoscope ................ 22

B. Hodoscope capabilities................. 23

C. Typical results ................. . . 24

1. Data correction .. . . . . . . . . . . . . . . 24

2. Data representation ............. 24

IV. AlTERNATIVE DIAGNOSTIC DEVICES .............. 30

A. Flash radiography ................ 30

B. Coded aperture .................. 31

C. Pinhole imaging ................. . . 32

D. In-core detectors . . . . . . . . . . . . . . 33

v. COMPARISON OF DIAGNOSTIC CAPABILITIES ............ 34

A. Theoretical considerations . . . . . . . . . . . . 35

1. Signal-response for fuel expansion and clad breach . . . 36

2. Detection sensitivity and figure of merit . . . . . . 37

3. Image-restoration techniques ............ 38

B. Direct comparison with pinhole systems . . . . . . . . . 38

1. Experimental data for PINEX-3 ........... 39

2. System qual ity indicators ............. . 40

3. Performance factors ............... 42

4. Conclusions from hodoscope/pinhole comparisons . . . . . 44

C. Indirect comparison with a coded-aperture system . . . . . . . 46

D. Flash radiography ................ 47

E. In-core detectors .................. 47

F. Comparative performance . . . . . . . . . . . . . . . 48

1. Specific diagnostic system quality indicators ..... 48

2. Analog data ................ 49

3. On-line data observation ............. 50

G. Optimization of performance ........... 50

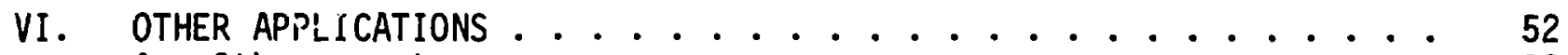

A. Other reactors ............... . . 52

B. Time-integrated radiography ............. 53

C. Reactor-power monitoring .............. 56

D. Ex-vessel monitoring ............... 57

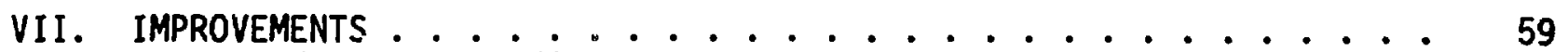

A. Two-dimensional diagnostics ............ 59

1. Hodoscope improvements planned............ 59

2. Other diagnostic techniques ............ 60

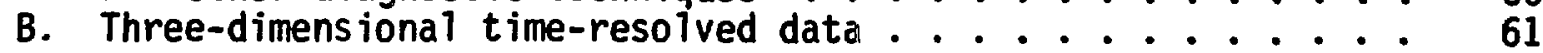

C. Three-dimensional static tomography .......... 61

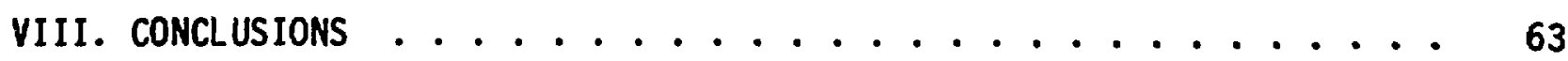

References..................... 66 
III-1. Hodoscope capabilities . . . . . . . . . . .

$\checkmark-1$. Demonstrated diagnostic system-quality indications for

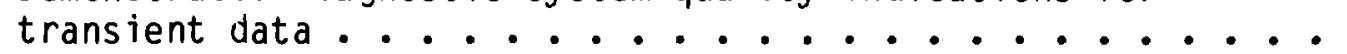

\section{LIST OF FIGURES}

I-1. Cross-section of test container for typical 7-pin subassembly

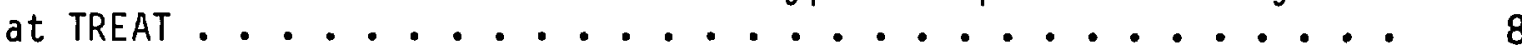

I-2. Schematic arrangement of test element and hodoscope at TREAT . . 9

III-1. Hodoscope scan of a single fuel pin while TREAT is held at $80 \mathrm{~kW}$ steady-state power................ 15

III-2. Schematic diagram of 1.2 m hodoscope system at TREAT . . . . 17

III-3. Photograph of $1.2 \mathrm{~m}$ hodoscope at TREAT . . . . . . . 18

III-4. Hodoscope collimator ray projections . . . . . . . . 18

IIJ.5. Detection-system packages for the TREAT hodoscope . . . . . 20

III-6. TREAT hodoscope electronic-system block diagrams . . . . . 21

III-7. Top view of hodoscope at CABRI/Cadarache . . . . . . . 22

III-8. Countrate as a function of time . . . . . . . . 25

II I-9. Intensity-modulated hodographs ............. 25

III-10. Symbol ic hodograph . . . . . . . . . . . . 26

III-11. Axial component of fuel motion ........... 2 ...

III-12. Multiparameter plot of hodoscope data from TREAT transient

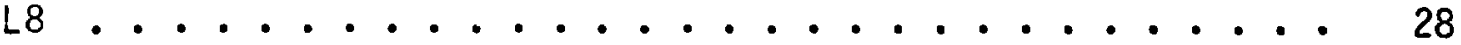

III-13. Worth-curve comparison .............. 28

III-14. Artist's reconstruction of PINEX-2 fuel movement . . . . 29

IV-1. Flash $X$-ray fuel-motion diagnostic method ......... 30

IV-2. Coded-aperture diagnostic instrument system (CAIS) at the

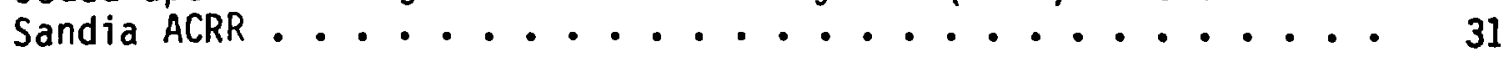


IV-3. Schematic arrangement of a pinhole-camera diagnostic imaging device tested at TREAT .................... 32

$V-1$. Simplified model of response functions ........... 35

V-2. Signal/background functional relationships . . . . . . . 38

VI-1. High-resolution hodoscope proposed for ETR (isometric view) . . 52

VI-2. Core layout for EBR-II modified to accept a coarse-resolution

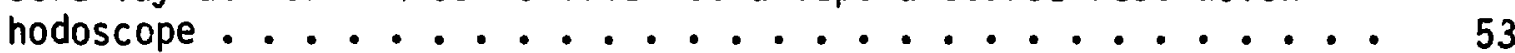

VI-3. Digital reconstruction of fast-neutron hodoscope scanning

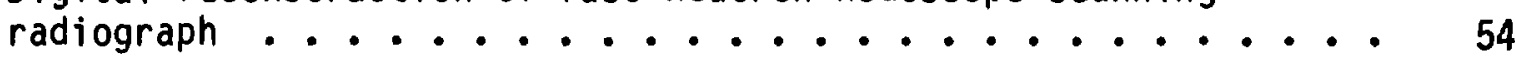

VI-4. Unconvoluted and deconvoluted scan-data comparison ..... 55

VII-1. Cross-section of planned 37-pin subassembly for TREAT-

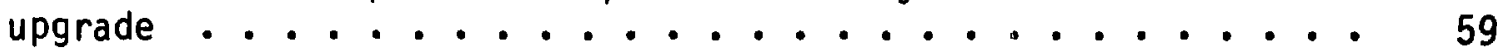

VII-2. Three-dimensional hodoscope cineradiography ........ 61

VII-3. Tomographic gamma-ray plenum scanning . . . . . . . . 62 


\section{ABSTRACT}

Nuclear and non-nuclear applications of cineradiography are reviewed, with emphasis on diagnostic instrumentation for in-pile transient-reactor safety testing of nuclear fuel motion. The primary instrument for this purpose has been the fast-neutron hodoscope, which has achieved quantitative monitoring of time, location, mass, and velocity of fuel movement under the difficult conditions associated with transient-reactor experiments. Alternative diagnostic devices that have been developed have not matched the capabilities of the hodoscope. Other applications for the fuel-motion diagnostic apparatus are also evolving, including time-integrated radiography and direct time- and space-resolved fuel-pin power monitoring. Although only two reactors are now actively equipped with high-resolution fuel-motion diagnostic systems, studies and tests have been carried out in and for many other reactors.

This report is more detailed version of a topical review invited by The Review of Scientific Instruments.

\section{INTRODUCTION}

The consequences of nuclear-reactor fuel-element failure have been the subject of extensive experimental and computational study. Postulated safety implications resulting from cladding failure and subsequent fuel motion are being evaluated worldwide in major experiment programs. of particular interest is the real-time behaviour of fuel elements when abnormally stressed due to hypothetical loss of coolant or sudden reactor overpower. Some of these unusual conditions are simulated in-pile (in nuclear reactors), and others can be tested out-of-pile (in laboratories away from a reactor).

For in-pile experiments, the test fuel is irradiated with a high-intensity burst from reactors usually designed for transient operation. The burst of neutrons and gamma rays induces fissions and other effects in the fuel and container. Experiments consist essentially of one or more fuel pins placed in a static container or coolant-circulation loop that is centered in the reactor core. Each test section (for example, Fig. I-1) is enclosed in multiple barriers that prevent radiation leakage. The vessels have been built to approach prototypical thermal-hydraulic and neutronic conditions for the fuel being tested. 
Extensive static and dynamic testing of fuel elements has taken place at many laboratories throughout the world. In particular, in-pile transient tests of fuel pins are conducted routinely at the United States Department of Energy react or TREAT in Idaho $[D I-61]$. Other U.S. reactors that subject test fuel to destruction are PBF, LOFT, ETR, and ACRR.

A wide variety of diagnostic techniques are utilized in monitoring nuclear-safety experiments. Some measurement devices are associated with the experiment, some with the host facility, and some with directview external instrumentation. The direct-view diagnostic instrumentation can image objects or their densities in static and time-resolved configurations, and they can measure extrinsic properties such as time- and spacevarying power distribution with the field of view.

This review concentrates on timeresolved radiographic (cineradiographic) measurement techniques (Sect. II) applied to in-pile nuclear-safety experiments. In-pile experiments must use more sophisticated instrumentation than out-of-pile because of the impact of the reactor facility. On the other hand, out-of-pile testing is more convenient to periorm, and valuable results with high-quality resolution are being achieved by diagnostic methods especially appropriate for the experiments. In recent years tests have focused on nuclear fuel for fast reactors; a special class of fuelmotion diagnostic instrumentation has been developed for use in these measurements. Because it is the only measurement system capable of yiewing the entire test assembly, direct-view instrumentation collects other data besides time-resolved fuel-motion information for in-pile reactor-safety testing.

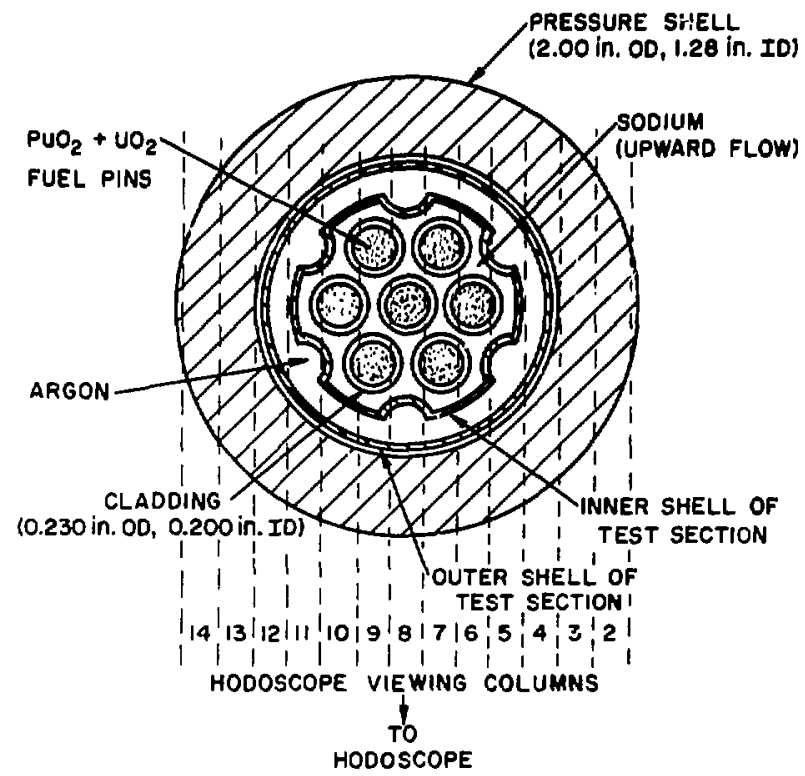

Fig. I-1. Cross-section of test container for typical 7-pin subassembly at TREAT. An additional thin secondary barrier surrounds the primary pressure shell, and the return leg (not shown) for sodium coolant flow might be positioned along the hodoscope line of sight.

A large proportion of the in-pile experiments are in support of fastbreeder-reactor development, for which fuel-motion safety issues are of great significance [WA-81]. A major goal of these measurements is the validation of computerized computational models of effects transferable to full-scale reactors under postulated accident conditions. In initial stages of transients, fuel motion internal to the pin cladding often takes place, culminating in some cases with failure of the cladding. Up to this point, 
the dominant motion of the fuel is constrained within definable boundaries. Subsequent to cladding breach, highly disorganized fuel motion can take place, including sweepout, eructation, slumping, etc.

Pre-failure fuel-motion detection, such as axial expansion, is desired, along with time and axial location of fuel breach through the cladding. For post-failure fuel motion, three-dimensional spatial resolution is usually not necessary because the axial reactivity change is the most desired information; the three most significant parameters are axial resolution, time, and fuel density. Diagnostic methods should be able to distinguish between steel and fuel by means that are more definitive than differences in the density. Without the time history of material motion during experiments, an extremely important aspect of the destructive experiments would be missing. It might not be possible to infer from the integral post-test data the instantaneous nodes that are important to reactivity calculations.

Two transient reactors in the United States (TREAT and ACRR) and one in France (CABRI) are uniquely arranged to accept high-resolution fuel-motion diagnostic instrumentation (Sect. III). The fuel being tested, which is placed at the center of the reactor core, emits fission and capture radiation induced by the burst of reactor neutrons; the emitted radiation can travel unimpeded through an opening in the core to the direct-view diagnostic instrumentation installed in the biological shield (Fig. I-2). The vertical height of the slot in TREAT is up to $1.5 \mathrm{~m}$.

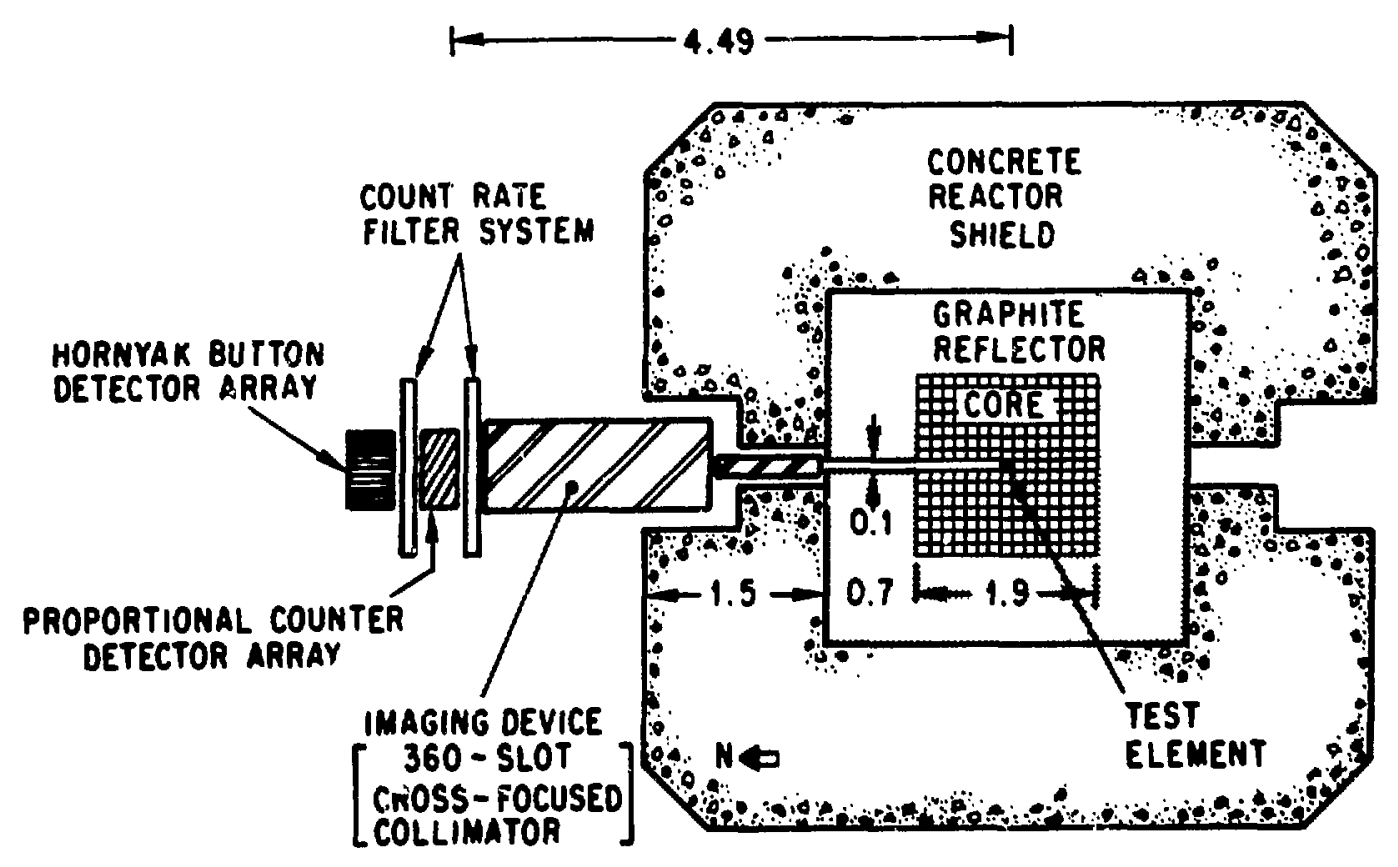

ALL DIMENSIONS IN METERS

Fig. I-2. Schematic arrangement of test element and hodoscope at TREAT. For most experiments the slot through the reflector and core terminates at the test-eiement container. 
Loss of reactivity and flux asymmetry introduced by the slot are not inconsequential; they can usually be partially compensated by adjusting the fuel loading in the core. A full slot through the TREAT core is worth about $5 \% \Delta k / k$ reactivity and a half-siot nearly $3 \%$. Usually the transient upper energy limit of the reactor is determined by conditions other than the slot worth.

The instrumentation task is defined by experimental conditions. Test fuel (from 1 to 37 pins) for in-pile experiments is normally placed in thickwalled $(1$ to $3 \mathrm{~cm})$ steel vessels or loops. The reactor may be directed into a brief high-power burst up to $20,000 \mathrm{MW}$ or persist at lower powers, say 100 MW for tens of seconds. In ACRR or CABRI, the full-width-at-half-maximum is just a few milliseconds, and in TREAT it could be as short as a few-hundred milliseconds. Because instrument signal strength is proportional to the reactor power, which during a transient may vary by a factor of $10^{4}$ or $10^{5}$, the instrumentation should have the dynamic range to produce useful data throughout the experiment. The diagnostic instrumentation is located out of the reactor core where it will not only survive the burst but also avoid undue background effects. Because of the brief duration of the transient, the recorded data is not analyzed until after the transient.

Out-of-pile experiments do not have to contend with a reactor environment. This eliminates the reactor background, reduces required experiment containment, and allows greater accessibility for instrumentation. Out-ofpile experiments usually cause fuel cladding or element failure by electrical or inductive heating [SP-82].

Except for calibrations and special non-destructive or threshold tests, the fission energy produced within the in-pile fuel capsule is usually sufficient to fail the fuel-pin cladding or induce expansion, melting, or other fuel-movement phenomena. Specific experiment objectives include measurement of the amount of fuel disturbed by the fission burst, the time and location of displacement, and the velocity of fuel. Diagnostic measurements should determine precise time and axial location of failure and specify the velocity and quantity of fuel relocation -- at time resolution of a millisecond or so, spatial resolution in the range of a millimeter horizontally and a centimeter vertically, and mass resolution in the order of a few hundred milligrams.

A relatively unique aspect of in-pile fuel-motion -ineradiography is the need for quantitative and dimensional data (Sect. $V$ ). Although qualitative phenomenological observation and visualization of processes is of importance, the precise and accurate determination of fuel-mass movement is the dominant objective. Achievement of the combined resolution in space, time, and areal density that is needed places unique demands upon the design and operation of the instrumentation that are not encountered in other forms of diagnostics. Moreover, compensation must be made for the nonuniform and time-varying nature of both the reactor power and its coupling to the fuel.

other instrumentation installed in the test vehicle is used for measurement of thermal-hydraulic conditions (flow, pressure, temperature). The physical remains of an experiment are examined afterwards by penetrating radiography and destructive disassembly. 
The iirst in-pile fuel-motion measurements were conducted soon after TREAT was finished in 1958, using cinephotographic techniques when it was possible to view the test section through a transparent window [G0-62]. Cineradiography and cinephotograpny are coniplementary in nature. Opticai wavelengths are such that only surface features are imaged in photography, but radiography extends from topographical mapping of subsurfaces to highly penetrating densitometry of volumes.

When plans were made to perform experiments with liquid sodium $a=$ a coolant and with optically opaque containers, development of a gamma-ray pinhole apparatus was undertaken starting in 1958 at the Naval Research Laboratory supported by Argonne National Laboratory. This concept gave way to a gamma-ray scanning system, which was tested at TREAT [BE-61]. Because the signal-to-background ratio achieved was too low, the method was not actively pursued beyond 1961 .

The fast-neutron hodoscope concept (Sect. III) was proposed in 1963, taking advantage of the experience gained in development of the gamma-ray systems. Tests performed in 1964 indicated a high signal-to-background ratio for fast-neutron detection. Consequently, the TREAT hodoscope system has evolved through several stages [DEV-75, DEV-77A, DEV-81a, DEV-82a] to its present advanced configuration.

Studies (Sect. VI) have been made of application of fuel-motiun diagnostics to other reactors. These include FARET, PBF, STF, ETR, EBR-II, ACRR, and other U.S. facilities [DEV-82d]. A fast-neutron hodoscope that incorporates sev'sral improvements in design and instrumentation has been installed and operated since 1978 in the CABRI reactor (Sect. III) at Cadarache under an internationally supported program. A four-channel hodoscope was built at Los Alamos National Laboratury for testing hodoscope applications to large test bundles [EV-79a, EV-79b, EV-80]. Pinhole diagnostic devices at TREAT and coded apertures at ACRR have been extensively tested (Sect. IV).

Because of the productive and sometimes essential role of fuel-motion diagnostics in reactor-safety experiments, the choice of an instrumentation system has considerable consequence. Design concepts of major safety-test reactors have centered about capability to tolerate high-resolution diagnostic systems. Selection of a diagnostic system for existing reactors can have substantial influence on development time, cost, availability, quality of data, and general utility. Without appropriate understanding of performance quality parameters, considerable effort can be nonproductive. Major sums of money have al ready been spent in studying applicability of alternative concepts of diagnostic systems for experiments in many reactors, for specific experiments in TREAT, for slot arrangements in an upgraded TREAT, for diagnostic calibration, and for future reactor designs.

Documentation of these studies is sparse and not formally published for the most part. Most of the diagnostic information was reported at four information excinange meetings, only two of which have been printed as government documents. Other data is accessible in national laboratory reports. Some relevarit information has been published in the transactions of three meetings on fast-reactor safety technology. 
Before reviewing existing and alternative fuel-motion diagnostic devices in detail, cineradiographic instrumentation applied more generally to nonnuclear and nuclear application will be surveyed briefly, particularly to provide understanding of limitations if applied to in-pile experiments. 


\section{CINERADIOGRAPHIC INSTRUMENTATION}

Cineradiography uses penetrating radiation to produce tine-resolved images. The detected radiation may emanate directly from the source (autoradiography), be attenuated by the subject (transmission radiography), or be induced in the subject (emissive radiography). The recoraing of radiographic projections is not limited to film or TV cameras, involving instead often other means of data collection and image reconstruction. Multi-dimensional information is often sought.

Although time resolution for real-time (0.02-0.06 s) reconstruction is sometimes adequate for technical applications, cineradiography al so embraces slow-motion (1-1000 s) and high-speed (0.01-0.0000001 s) events. Time resolution is usually sought to be commensurate with repetition rates, frequencies of occurrence, or source pulsing. For technique evaluation, a single radiograph, especially one of short duration, falls within the field of interest. The ultimate goal, as in cinephotography, is a sequence of slow-motion, real-time, or time-lapse images repiayed to illustrate changes.

A status report on some radiation sources for cineradiography has been prepared by Polansky [PO-80] and by John, Dance, and Whittemore [J0-82]. Detectcr and imaging systems have been reviewed by Halmshaw [HA-80], Kuhl [KU-80], Bates [BA-80], Chalmeton [CH-80], Jacobs [JA-80], Kingsley [KI-80], and Bossi and 0ien [BOS-81a, BOS-81b].

Examples of industrial applications include dynamic imaging of gas turbine operation using $x$-ray transmission and enyine lubrication dynamics using cold-neutron transmission [ST-80, PU-81]. Real-time imaging of pipe welding has been accomplished [RU-80], and studies in Japan of beam welding and cutting have been reported [AR-81]. Real-time neutron-radiographic inspection techniques for aircraft wing structure have been developed [PA81]. Basic limits in real-time radiographic systems have been analyzed by Kuriyama, Boettinger, and Burdette [KUR-80]. Imperfections in crystals [HA80; CHI-80; DA-80] have been mapped by real-time topographic methods.

A major concentration of development has involved military applications, especially ballistics and explosives research. Heapons-research practices in France $[H A-81, J A-81]$, the United States [DI-81,BR-81], the United Kingdom [BL-8] ], the Federal Republic of Germany [EB-81], and Sweden [MA-81] were descricad at the First European Conference on Cineradiography. Fissionexplosive testing has been time-imaged by pinhole detectors, although little information: is published.

The use of cineradiography in physical measurements of motion, flow, spark discharges in liquids, and laser-produced plasmas has been reported. other major uses of cineradiographic techniques are found in biophysics and medicine. Real-time fluoroscopy and radiography have been practiced for some time [ME-80, ZI-80, SC-80, KR-80], and a three-dimensional time-resolved whole-body s;canner is operating at Mayo Clinic [HAR-81, RI-80] in the United States. Image-formation improvements associated with diagnostic radiology include beam filtration [SI-80], computerization [SK-80], and automation [LA80]. Many developments in computerized axial tomography that have resulted from medical research are applicable to time-resolved radiography [HAR-81]. 
Most nuclear applications involve studies of fission and fusion reactor fuel. Some of the experimental work is done out-of-pile while other work is much more closely associated with the radiation source. Fission-fuel development takes place in many industrialized countries, and substantial facilities have been dedicated to either neutron [H0-81] or $x-$ ray [SP-82, WI81] cineradiography of fuel elements in out-of-pile testing.

Fusion micro-explosion and plasma diagnostics have been carried out [LA$81, \mathrm{CH}-81, \mathrm{RU}-81$ ] by related diagnostic techniques.

The mobility of the test object and its prototypic test environment are major factors in utilization of facilities that specialize or have unique capabilities in conventional radiography. Although in-pile nuclear-reactor testing generally cannot be conducted in these facilities, other cineradiographic techniques can be carried out taking advantage of highenergy beams of neutrons or photons and of mu?ti-dimensional detectors. Examples of such special facilities are PHERMEX [CI-81], Saturne, CERN [SA81], IPNS [STR-81], and a new high-explosive $x$-ray flash-radiography facility at Livermore. The Argonne IPNS can also provide time- and energy-resolved radiographs, similar to techniques demonstrated at the NBS Linac [SC-81].

In-pile testing of fission fuel, primarily for the nuclear-reactor safety program, is the application that th:s review concentrates upon. Hodoscopes (multichannel imaging systems), pinhole cameras, coded apertures, and in-test or in-core detectors have been used or developed as fuel-motion diagnostic devices.

The demands upon in-pile diagnostics instrumentation are more severe than applications in out-of-pile reactor experiments, which, lacking competing background, can make use of pulsed or continuous $x$-ray sources of sufficient penetration. Relatively straightforward detection and image-recording systems may be used out-of-pile.

In fact, it is the nature of the background and test surroundings that distinguishes fuel-motion diagnostics from other forms of cineradiography. These conditions have particularly governed the choice of penetrating radiation to be detected. 


\section{HODOSCOPE DIAGNNOSTIC INSTRUMENTATION}

The fast-neutron hodoscope [DEV66] is in essence a multichannel system of collimation, detection, and recording of fission neutrons emitted from nuclear-reactor fuel within the field of view. Background is a combination of outscattering from the test container of neutrons originating within the fuel and by in-scattering from the test container of neutrons from the reactor core. Advantaye is taken of the fact that fast neutrons usually lose energy when scattered away from direct trajectories. The collimator slots confine accepted radiation to a very small acceptance angle $\left(10^{-5}\right.$ sr). Neutrons that have lost significant energy are not counted; as a result, the signal-tobackground ratio for hodoscope detectors is typically in the range of 0.5 to 4 (Fig. III-1).

The first cperational hoduscope in 1965 had a 334-channel collimator capacity, but was instrumented with only 50 detectors and used an analog photographic data-recording system. Its field of view was $0.06 \times 0.5 \mathrm{~m}$. Qualitative fuel-motion data and quantitative time and spatial identification were achieved. operation was sufficiently successful to point the way to improvements and to authorize full instrumentation. In 1969 the analog data-recording system was replaced with a digital photographic system [DEV-75a] that had wide-band capacity, resulting in useful quantitative fuel-mass results.
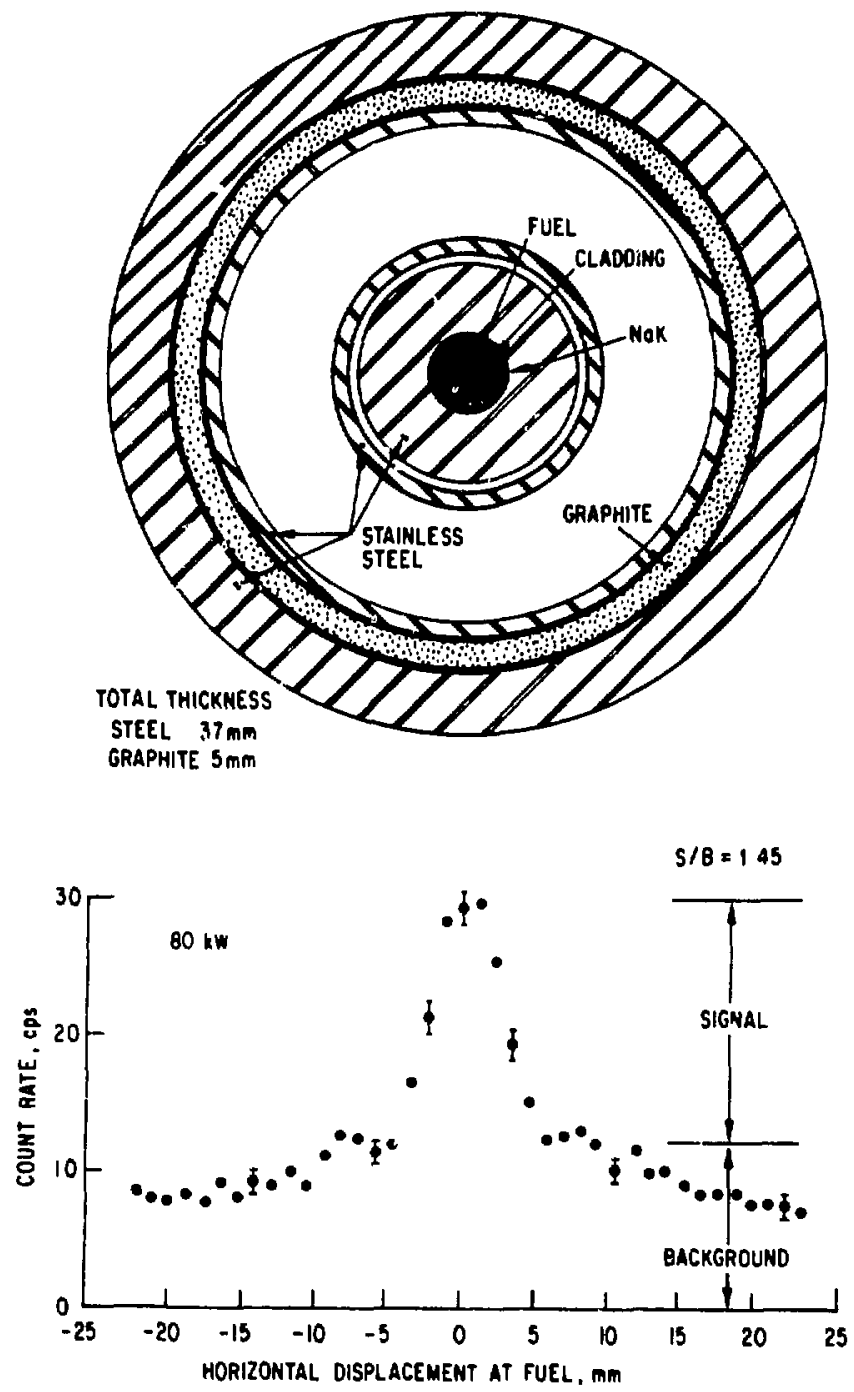

Fig. III-1. Hodoscope scan of a single fuel pin while TREAT is held at $80 \mathrm{~kW}$ steady-state power. The collimator is displaced in $\mathrm{mm}$ increments, and the count rate of the detector is recorded for each position. Data from all detectors is recorded simultaneously, but only the data from one Hornyak button detector is plotted here.

Since 1969, the TREAT hodoscope has been operated in over 120 major tests. In 1975 a 360-channel collimator was built to view a larger field that would match experiments with longer, more prototypical fuel pins. In 1976 a stateof-the-art magnetic-disk system [DEV-76a] was installed as the primary datarecording medium, with both magnetic tape and the older digital photographic systems operating in a redundant recording mode.

Dedicated interactive computing facilities for data analysis and display have been in use since about 1970. Additional developments have included a 
semi-automatic horizontai scanning capability, which permits the accumulation of time-integrated fast-neutron radiographic images of moderate resolution. Most recently, remote-controlled vertical and horizontal scanning have been implemented, along with a tandem arrangement of tw: rasi-noutron detectors.

The CABRI hodoscope [BL-76a, BL-76b, HE-82], though deve']oped al ong the same principles as the TREAT hodoscope, has several significantly different features. It achieves more precise static location of fue? by careful design, machining, and detector selection. Published results have sho:an it has reached design goais for high accuracy in measurement of axial fuel motion and exceeded goals for radial motion.

\section{A. Description of apparatus}

1. TREAT hodoscope (Fig. III-2)

The present TREAT hodoscope collimator subsystem [FI-82b] basically consists of a stack of tapered steel plates with 360 milled angled slots. The steel plates (Fig. III-2) are encased in concrete for shielding. A pivot system allows lateral translation of the collimator. Neutrons that originate in line of sight of a collimator slot are able to pass freely through the collimator; neutrons that intersect the steel have less than $1 \%$ chance of reaching detectors at the emerging face of the collimator.

Figure III-3 is a photograph of the collimator/detector system at the TREAT reactor. Electro-mechanical systems exist for horizontal pivoting of the collimator to perform the scanning operation mentioned previously and for positioning a neutron source in front of each detector for checkout and calibration purposes. A lead (Pb) filter is used to reduce the effect of gamma rays. The fast-neutron detectors used in the hodoscope are relatively insensitive to gamma fluxes. Figure III-4 traces collimator ray lines from the object plane to the detector image plane.

A rectangular area $1.2 \mathrm{~m}$ high and $6.7 \mathrm{~cm}$ wide at the center of the reactor is viewed directly by the 360-channel colitimator, which is located about $3 \mathrm{~m}$ from the fuel being tested. The collimator-slot transfer function overlaps with adjacent horizontal channels but not with vertical channels.

Two types of fast-neutron detectors are attached in tandem at the end of each slot. These detectors are relatively efficient for fission neutrons, while being highly insensitive to gamma rays. The detectors also have a wide dynamic range, having low noise level and capability to achieve over $10^{5} \mathrm{c} / \mathrm{s}$. 


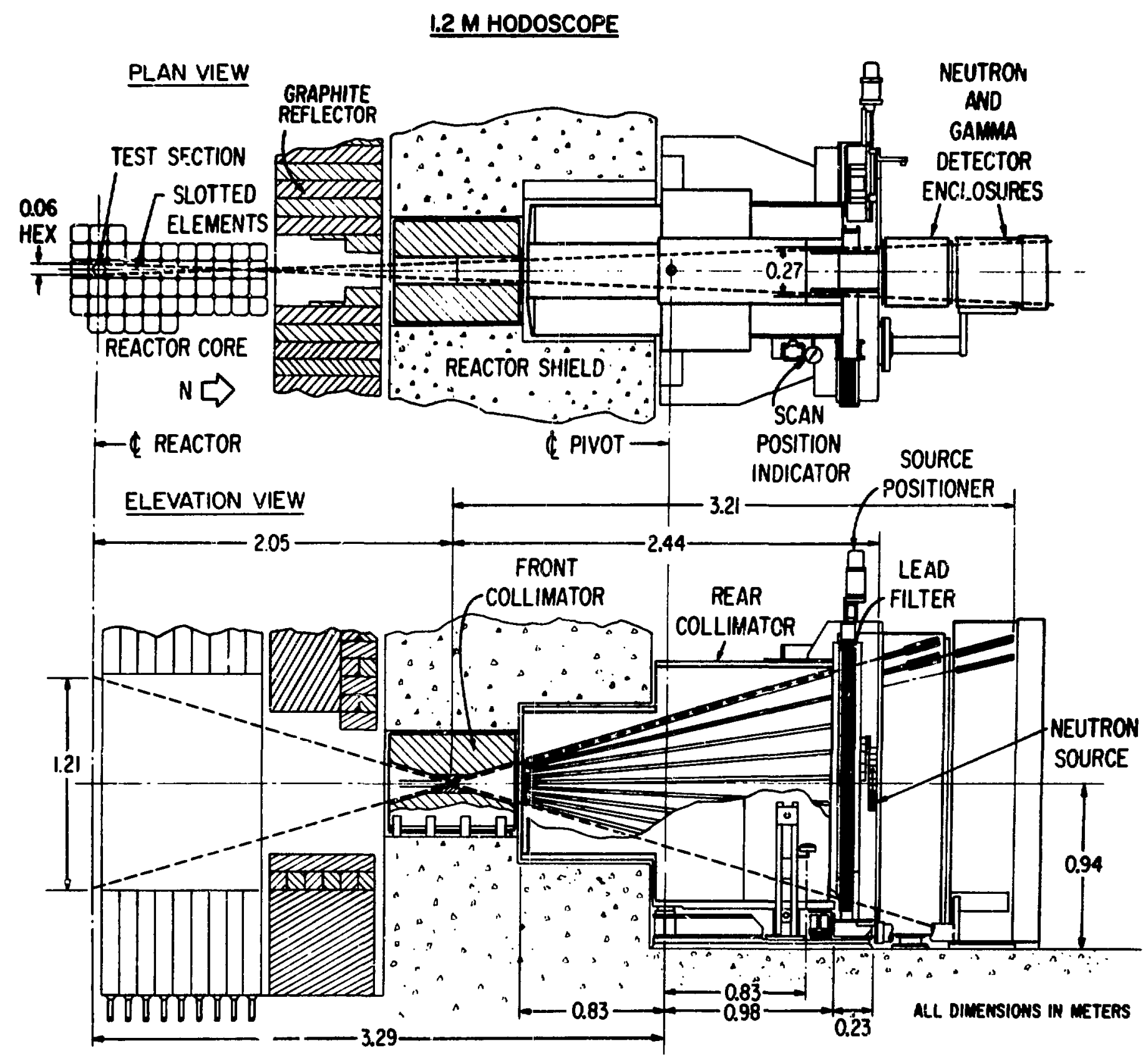

Fig. III-2. Schematic diagram of $1.2 \mathrm{~m}$ hodoscope system at TREAT. Two fastneutron and one gamma-ray detector can be mounted in tandem at the end of each slot. The front collimator acts as shielding rather than as a pinslit imaging component. 


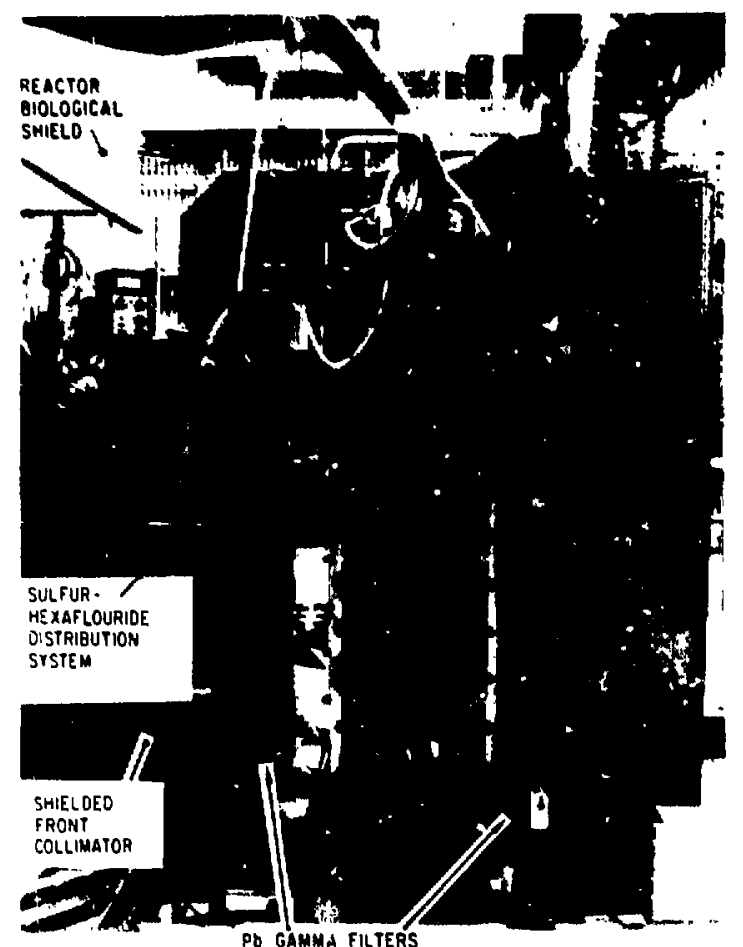

Fig. III-3. Photograph of $1.2 \mathrm{~m}$ hodoscope at TREAT. Lead (Pb) filters reduce effect of gamma rays and attenuate neutrons to avoid countrate saturation; calibration apparatus is remotely controlled from a computer to position a neutron source reproducibly in front of each Hornyak-button detector.

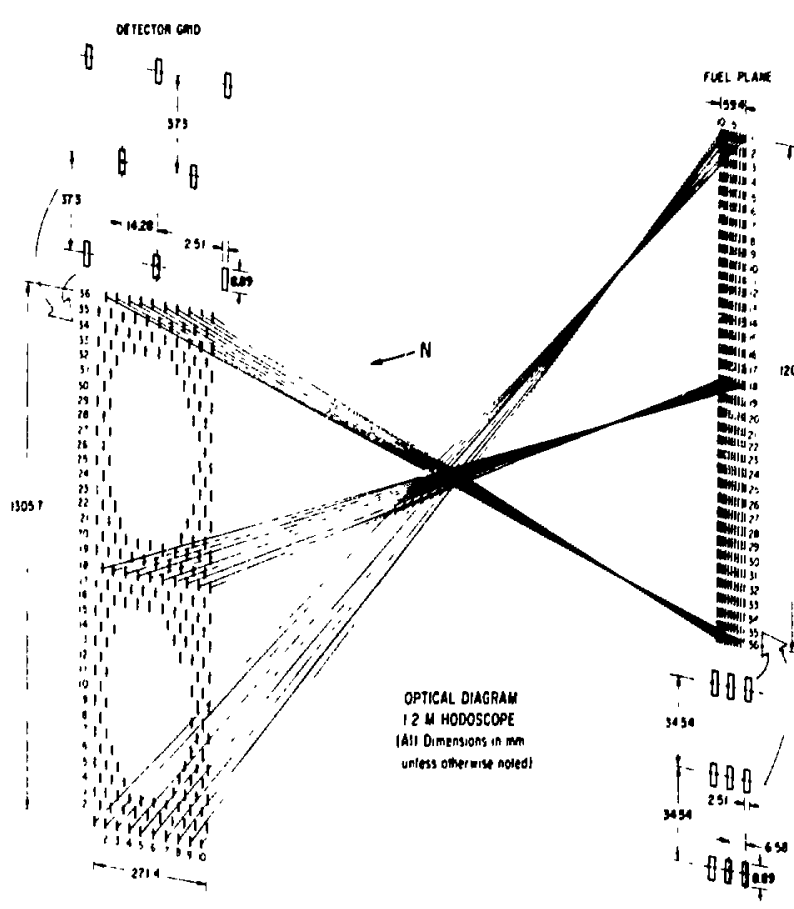

Fig. III-4. Hodoscope collimator ray projections. Each pixel on the fuel plane is mapped by individual collimator channels onto assigned detectors at the detector grid. The ray projections cross both vertically and laterally in order to optimize available space.

One of the neutron detectors (Fig. III-5a) is a proton-recoil scintillator known as a Hornyak button [H0-52, EV-79b, FI-82a]. Scintillation flashes in the Hornyak buttons are amplified in photomultiplier tubes and conveyed to an electronic circuit board, one for each collimator channel. After pulse shaping and amplification, a standard pulse is issued from a discriminator.

The other type of neutron detector (Fig. III-5b) is a methane gas-filled proportional counter [FI-80] adapted from a design proven for the CABRI hodoscope [BL-76a]. This array of high-pressure (5 atm) detectors has undergone extensive steady-state and transient proof tests. Compared to the Hornyak-button scintillators, the gas proportional counters are more linear at high power, have higher efficiency, are more stable, and are less susceptible to noise -- at an acceptable tradeoff in signal/background ratio.

A sodium-iodide detector (2.54 cm length $\times 1.87 \mathrm{~cm}$ dia.) to be used for detection of steel movement is also mounted in tandem behind the neutron detectors. Fig. III-2 illustrates the schematic relationship of these detectors. 
Electronic scalers assigned to each channel collect counts for the duration of each counting interval until the data is transferred to a dedicated high-speed magnetic disk for permanent recording (Fig. III-6a and b). The scalers are immediately reset and the count begins again automatically. This data-recording system can make 8000 recordings of the contents of 360 twelve-bit scalers at intervals as short as $0.1 \mathrm{~ms}$. A typical recording cycle is $2 \mathrm{~ms}$ for all detectors. A second data-recordili? system of similar design but different components has recently been installed for greater capacity and added redundancy.

Control computers and on-line test points are used in conjunction with the recording system. In view of the large amount of data generated, various data transfer and processing systems are invoked. Analysis of the data requires data processing, image reconstruction, and some interpretation.

Normalization must take place for instantaneous power level and for nonuniform efficiency of detectors. Corrections are applied for instrumental effects, such as deadtime and nonlinear response. Background is subtracted, based on calibration experiments and curve fitting. Compensation for inherent effects such as self-shielding and axial neutron-flux gradient must be undertaken in order to quantify the fuel-mass displacement results [RH-79, DEV-82a]. 

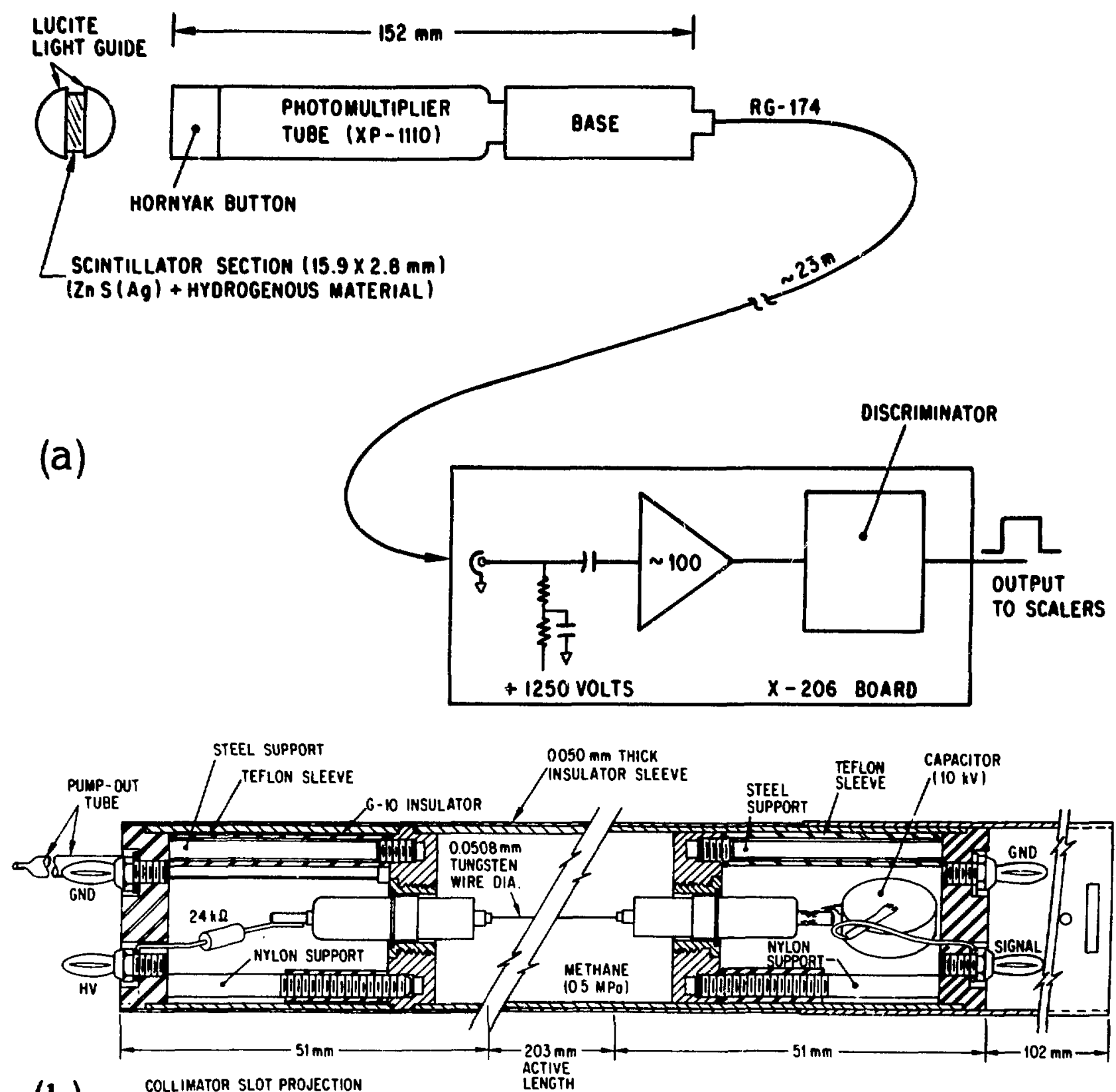

(b)
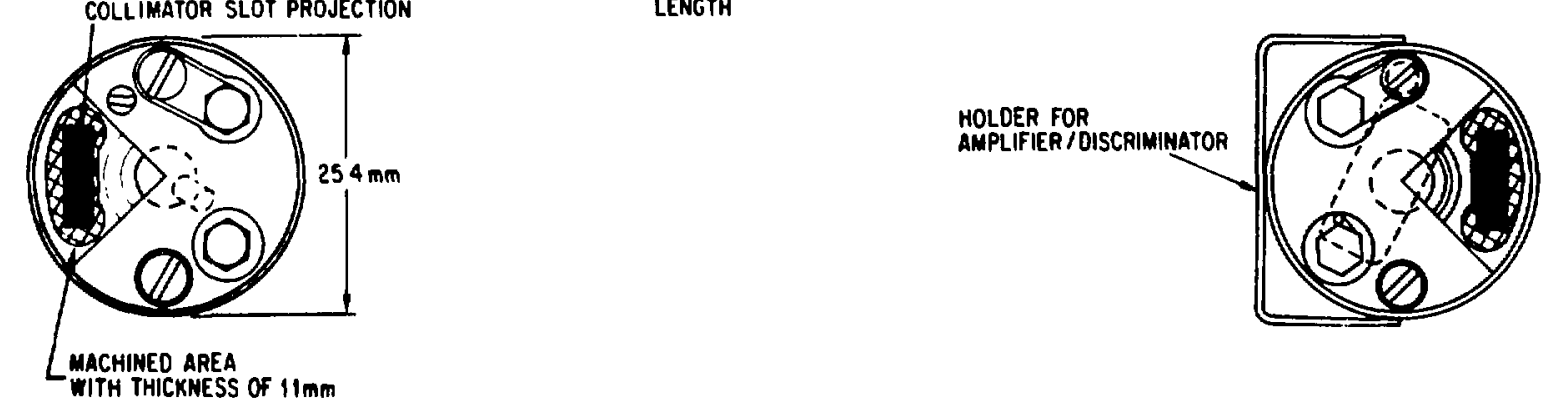

Fig. III-5. Detection-system packages for the TREAT hodoscope. (a) Hornyakbutton fast-neutron scintillation detector mounted on photomultiplier tube; linear signal is sent by coaxial cable to amplifier/discriminator board. (b) Proportional-counter assembly, showing offset (darkened) region where collimated beam passes so as to minimize interaction with detector structure; miniature amplifier/discriminator board is mounted directly at the rear of the counter. 


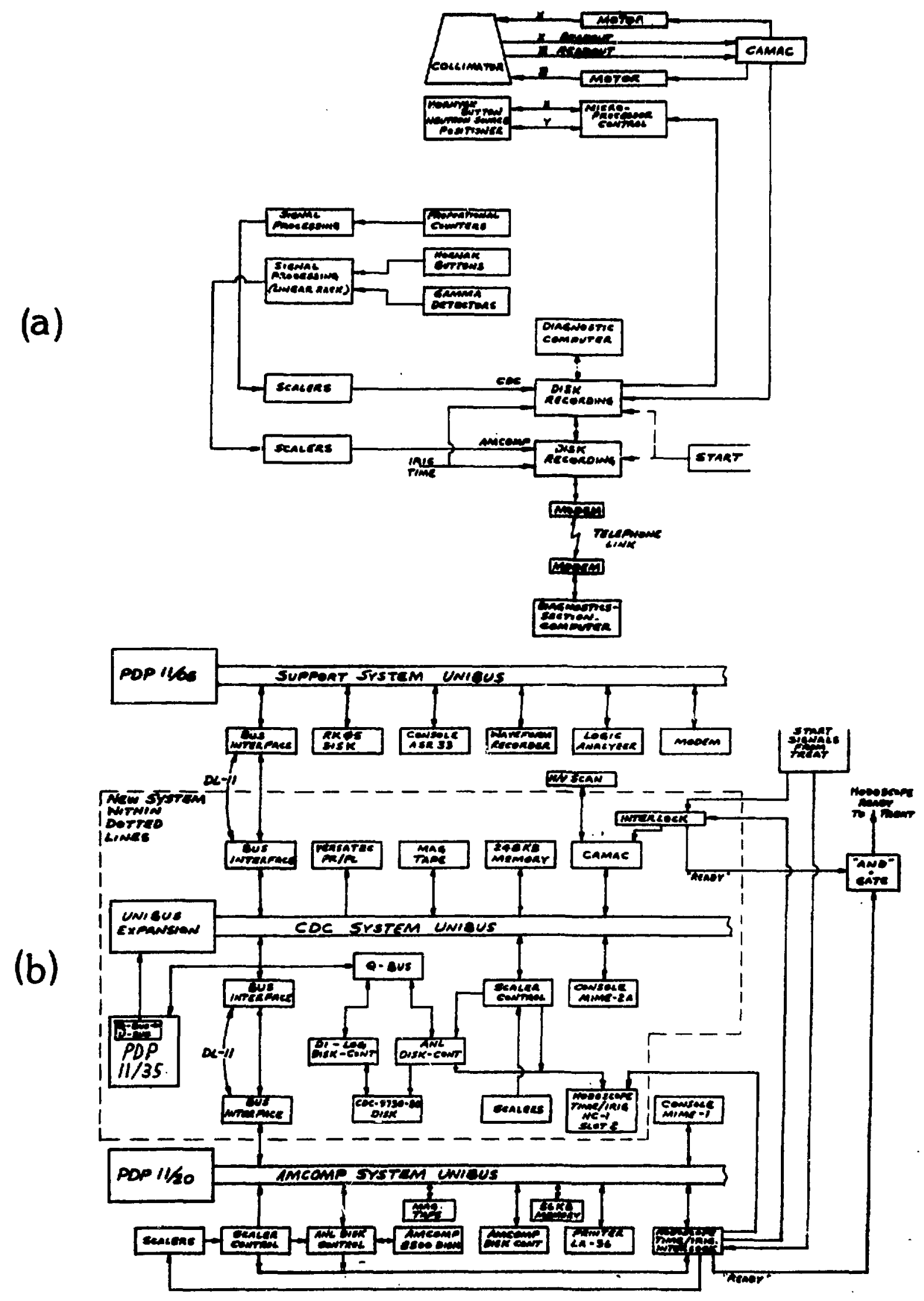

Fig. III-6. TREAT hodoscope electronic-system block diagrams. (a) Overall system diagram, depicting data pathways from detectors to recording system and collimator movement controls; telephone link is used for off-line data transmission from TREAT to Argonne, Chicago. (b) Digital portion of hodoscope data-acquisition system, with data stored on CDC and AMCOMP headper-track disks and backed up on magnetic tapes. 


\section{CABRI hodoscope}

The hodoscope collimator at CABRI (Fig. III-7) is made of iron plates, 300 $\mathrm{cm}$ in length, in 3 columns, each with 51 rows of detectors. Slot dimensions are $1 \mathrm{~cm} \times 2 \mathrm{~cm}$ at the collimator outlet and $0.75 \mathrm{~cm} \times 1.5 \mathrm{~cm}$ at the entrance.

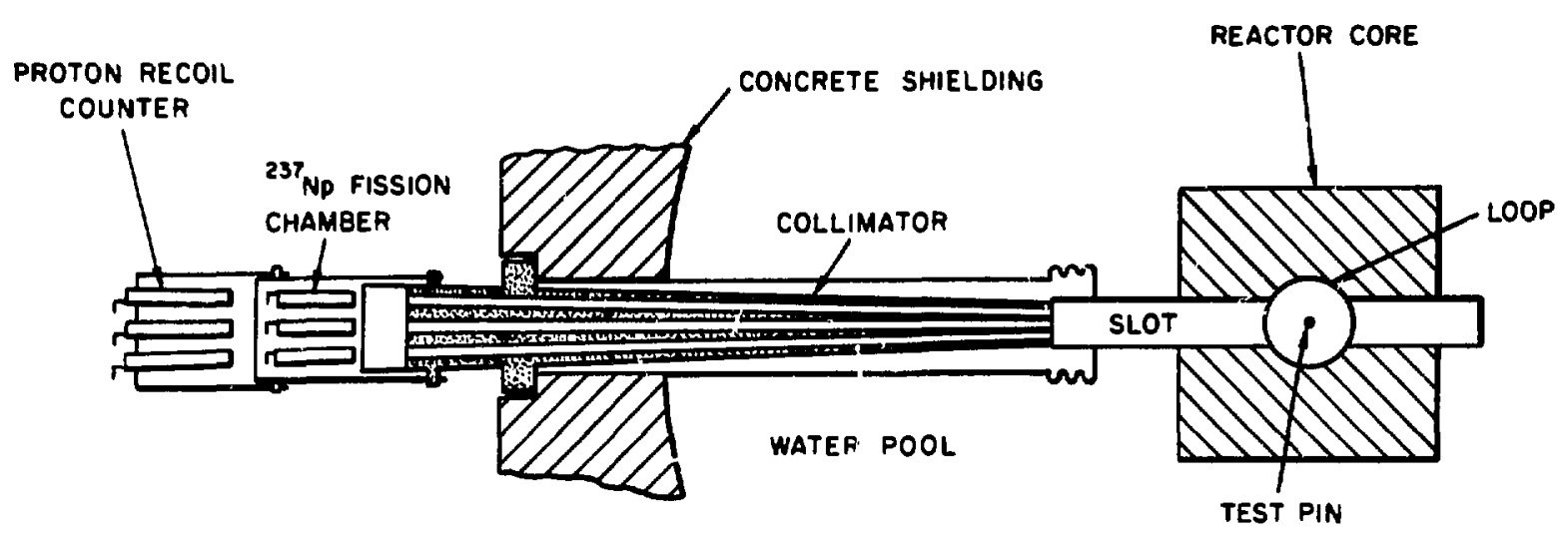

CABRI

Fig. III-7. Top view of hodoscope at CABRI/Cadarache. There are 51 rows of detectors in each of the three columns, and two types of detectors are shown in tandem behind each of the 153 collimator slots. The test pin is surrounded by its own containment vessel. As shown, the slot is clear of fuel straight through the core.

Two types of neutron detectors are mounted in tandem: fission counters and proton-recoil gas proportional counters. Each fission coynter agray consists of 100 plates normal to the beam, each plate with $1 \mathrm{mg} / \mathrm{cm}^{2}$ of $237_{\mathrm{Np}}$, having a fission neutron efficiency of $6 \times 10^{-4}$; pulse-pair resolution is $75 \mathrm{~ns}$. The proton-recoil counter is $20 \mathrm{~cm}$ in length, $2.54 \mathrm{~cm}$ dia., with efficiency adjustable between $10^{-2}$ to $10^{-3}$; pulse-pair resolution is 250 ps. Count-rate limits are $7 \times 10^{6} \mathrm{c} / \mathrm{s}$ for each fission counter and $3 \times 10^{6} \mathrm{c} / \mathrm{s}$ for each proportional counter.

Signal processing and recording is separated by optically isolated gates. 320 12-bit scalers record detector data, which is buffered into a 14-track analog magnetic tape system by pulse-code modulation techniques. A1 discriminator levels can be set remotely by a minicomputer via digital-toanalog converters. 
Signal/background ratio for a single pin is about 0.4 to 0.5 , lower than in TREAT because of differences in detector thresholds.

\section{B. Hodoscope capabilities}

Demonstrated key capabilities of the TREAT [DEV-82a] and CABRI [B0-79] hodoscopes are summarized in Table III-1.

\section{TABLE III - I}

\section{Demonstrated Hodoscope Capabilities}

\begin{tabular}{|c|c|c|}
\hline $\begin{array}{l}\text { Maximum viewing height } \\
\text { Maximum viewing width }\end{array}$ & $\begin{array}{l}1.2 \mathrm{~m} \\
7 \mathrm{~cm}\end{array}$ & $\begin{array}{l}1.0 \mathrm{~m} \\
3 \mathrm{~cm}\end{array}$ \\
\hline Collimator slots & 360 & 151 \\
\hline $\begin{array}{l}\text { Interchannel horizontal spacing } \\
\text { Interchannel vertical spacing }\end{array}$ & $\begin{aligned} 7 \mathrm{~mm} \\
34 \mathrm{~mm}\end{aligned}$ & $\begin{array}{l}10 \mathrm{~mm} \\
20 \mathrm{~min}\end{array}$ \\
\hline $\begin{array}{l}\text { Detectable horizontal motion } \\
\text { Detectable vertical motion }\end{array}$ & $\begin{array}{l}0.2 \mathrm{~mm} \text { (minimum) } \\
6 \mathrm{~mm}\end{array}$ & $\begin{array}{l}0.2 \mathrm{~mm} \\
2 \mathrm{~mm}\end{array}$ \\
\hline $\begin{array}{l}\text { 1-pin mass-displacement resolution } \\
7 \text {-pin mass-displacement resolution }\end{array}$ & $\begin{array}{l}0.05 \mathrm{~g} / \text { channel (minimum) } \\
0.35 \mathrm{~g} / \text { channel (minimum) }\end{array}$ & $\begin{array}{l}0.05 \mathrm{~g} / \\
\text { channel }\end{array}$ \\
\hline $\begin{array}{l}\text { Data-collection intervals } \\
\text { Data-interval capacity }\end{array}$ & $\begin{array}{l}0.1 \mathrm{~ms} \text { (minimum) } \\
2 \mathrm{~ms} \text { (typical) } \\
8000 \mathrm{intervals} \text { (maximum; } \\
3600 \mathrm{~s}\end{array}$ & $9000 \mathrm{~s}$ \\
\hline $\begin{array}{l}\text { Minimum react or oower } \\
\text { Maximum reactor power } \\
\text { Dynamic-power range } \\
\text { Power-coupling range }\end{array}$ & $\begin{array}{l}10 \mathrm{~kW} \\
20,000 \mathrm{MW} \\
10,000 \\
1-6 \mathrm{~J} / \mathrm{g}-\mathrm{MW}\end{array}$ & $\begin{array}{c}0.2 \mathrm{~kW} \\
10,000 \mathrm{MW} \\
100,000 \\
6 \mathrm{~J} / \mathrm{g}-\mathrm{MW}\end{array}$ \\
\hline
\end{tabular}

Notes

a. For $1.2 \mathrm{~m}$ collimator; a smaller $0.5 \mathrm{~m}$ collimator is less frequently used. b. A recently added system has 30,000-interval capacity (at 1 ms minimum).

The results of Table III-l are generally best values which cannot necessarily be simultaneously achieved. For example, space and time resolution are inversely related to each other because of statistical limitations.

At a coupling factor of $1 \mathrm{~W} / \mathrm{g}-\mathrm{MW}$ of TREAT power, the hodoscope signal/ background ratio would be about 0.5 ; a loss of $1 \mathrm{~g}$ out of $4 \mathrm{~g}$ of original 
fuel would be detectable at $0.05 \mathrm{~J} / \mathrm{g}$, and a loss of $0.4 \mathrm{~g}$ out of $4 \mathrm{~g}$ would require about $7 \mathrm{~J} / \mathrm{g}$.

Spatial resolution for most practical modes of fuel motion in TREAT is far better than the interchannel spacing, which is $34 \mathrm{~mm}$ vertically and $7 \mathrm{~mm}$ horizontally. In practice, vertical resolution of fuel expansion of about 6 $\mathrm{mm}$ is routinely achieved. Fuel motion within annular fuel having a central hole diameter $0.8 \mathrm{~mm}$ has frequently been detected and reported (see Fig. 18 of $[D E V-82 a])$.

Additional information on material motion has been sought from experiments. Fuel, structural material, and coolant are the primary constituents of a typical test assembly. The fuel is usually enriched uranium or plutonium, the structure is made of steel, and the coolant is liquid sodium. Gamma-ray detectors have been added in tandem at the TREAT hodoscope and operated to detect 6 to $10 \mathrm{MeV}$ capture gamma rays emitted from the components of steel [DEV-76c, FI-76, FI-77]. These gamma rays are thus indicative of the position of the steel during a test. Were it not for the large amount of uninvolved steel of the test container, the $0.4 \mathrm{~mm}$ steel cladding of the fuel might be detectablc. According to results achieved so far, detection of steel movement takes place when enough is accumulated to cause a flow blockage.

The boiling of sodium during safety experiments causes reactivity effects that justify direct spatially resolved measurement. This is a difficult task that has been the subject of investigation, with very limited success [DEV$76 d]$.

\section{Typical results}

1. Data correction

Data is corrected [DEV-75a, DEV-76a, DEV-82a] for deadtime, efficiency, and nonlinearity. Reactor-power amplitude is cancelled out by normalization through detector arrays. Axial flux gradients are accommodated. The initial portion of a transient, where a known quantity of fuel is present, is used for calibration of the fuel motion.

\section{Data representation}

The experimental data is routinely reconstructed into various image reconstructions of the original events. Because applications of hodoscope data require precise knowledge of the fuel mass involved in each displacement episode, much of the ensuing processing and analys is is devoted to quantification of the data [DEV-82a]. Consequently, the results which are presented below are selected to show a sample of the techniques of illustration and quantification. Such results are usually embodied in a series of internal reports that describe all aspects of the data treatment; eventually articles that contain a summary of data accumulated and analyzed are published by the experimenter [SI-81]. 
Any detector in the hodoscope array responds to changes in fission density, which is directly related to fuel density and power level. Figure III-8 contains graphs of the power-normalized count rates of several detectors plotted as a function of time into the transient. Changes that take place when statistical significance becomes adequate (after steady-state power plateau in this case) are indicated by changes in the normalized count ratio. Several methods of power normalization are available, including the use of detectors focused on parts of the array that are unlikely to see fuel motion.

The data from all detectors may be displayed in ways that give access to an integrated view of the course of fuel motion. Figure III-9 contains intensity-modulated renditions (hodographs), in which grey-level density equates directly to higher fuel mass. The fuel pin is seen to undergo twodimensional snaking that corresponds to a helical spiral in three dimensions.

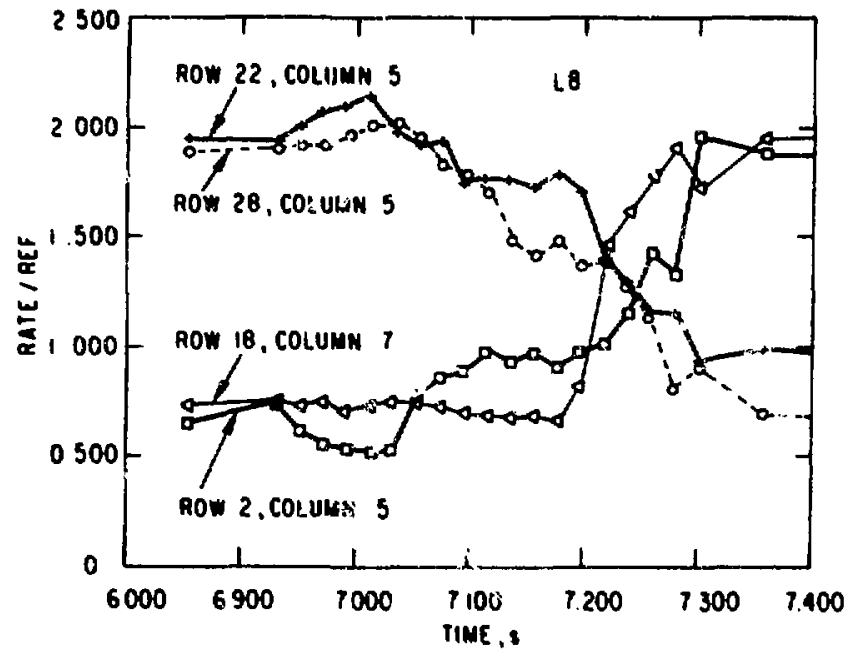

Fig. III-8. Countrate as a function of time. A decrease in countrate in Row 7 indicating a substantial slumping of fuel from above coincides with loop instrumentation data indicating an abrupt increase in clad melting. Then at atsut 27 $s$ into experiment $L 4$ a vigorous eructation dispersed that fue 1 up and out of the channel view.

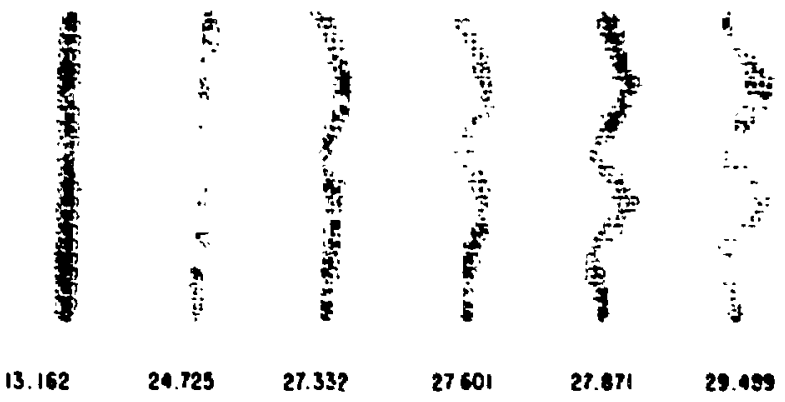

Fig. III-9. Intensity-modulated hodographs. During the course of the R3 transient, power was added while the single fuel pin was deprived of sodium coolant. As a result, the fuel pin underwent helical bending (by $24.725 \mathrm{~s}$ ) and loss of cladding restraint (by $27.332 \mathrm{~s})$. At the end of the transient $(28.489 \mathrm{~s})$, the fuel pellets were braced by the primary container (not shown). 
For quantitative use, a symbolic presentation form has been developed. Figure III-10 contains four samples from an extensive sequence of symbolic hodographs that illustrate the conversion to fuel mass estimates.

In reactor-safety studies, the axial component of fuel motion is frequently the property of main interest. In that case, the results of a symbolic hodograph may be summed across rows to produce a set of row-average column results, as chrivn in Fig. III-11. The axial fuel motion trend for that experiment can be clearly seen in a quantitative manner. In these plots only changes from the reference fuel condition have been plotted, so that grey areas represent no change, dark areas are net gains, and light areas are losses of fuel.

A way of presenting the large amount of data in a manner that can be compared to nuclear-reactor accident analys is codes is given in Fig. III-12. In this mult iparameter example, the axial fuel profile is plotted along a time axis. The data may also be represented by a single parameter, reactivity-worth changes as a function of time, converted to expected behaviour of larger subassemblies in a power reactor (Fig. III-13).

Finally, movies can be made to convey the dynamic aspects of the experimental results. We have done this in a number of cases, and Figs. III-9 and III-14 show some of the sequences involved.

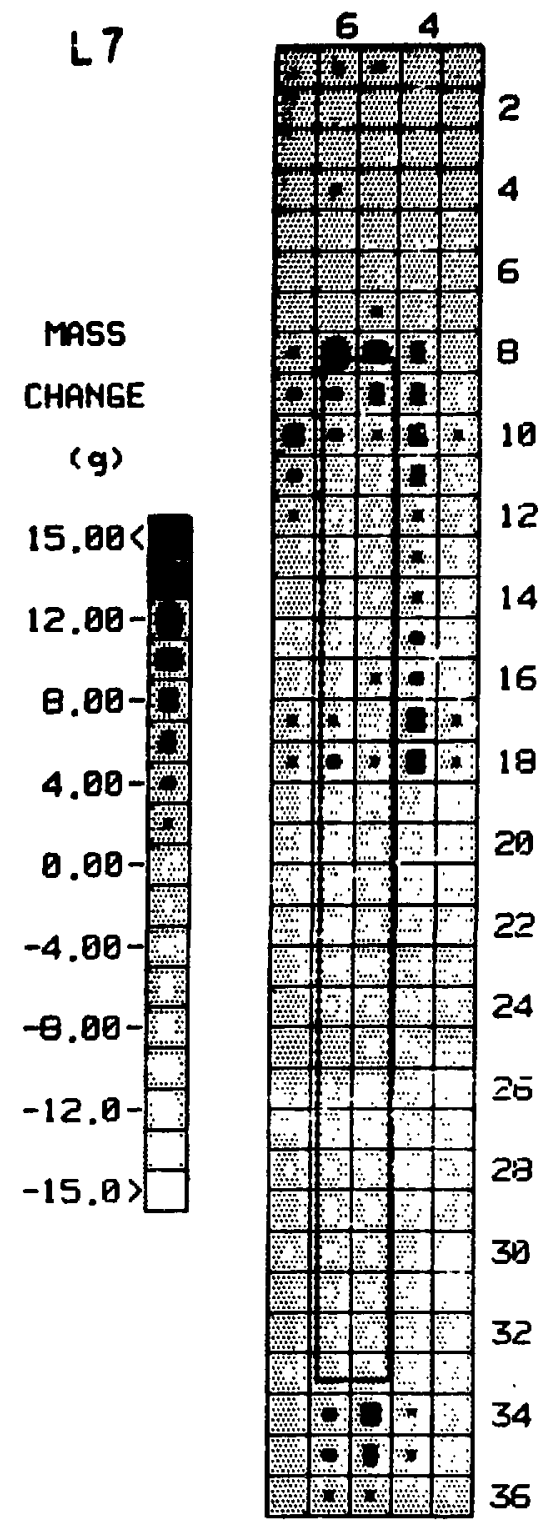

\section{$15.543 \mathrm{~s}$}

Fig. III-10. Symbolic hodograph. The dark symbols indicate scaled gains and the light symbols losses in transient at $15.543 \mathrm{~s}$ compared to original fuel distribution. Fuel has moved axially and radially away from the original rectangle. 


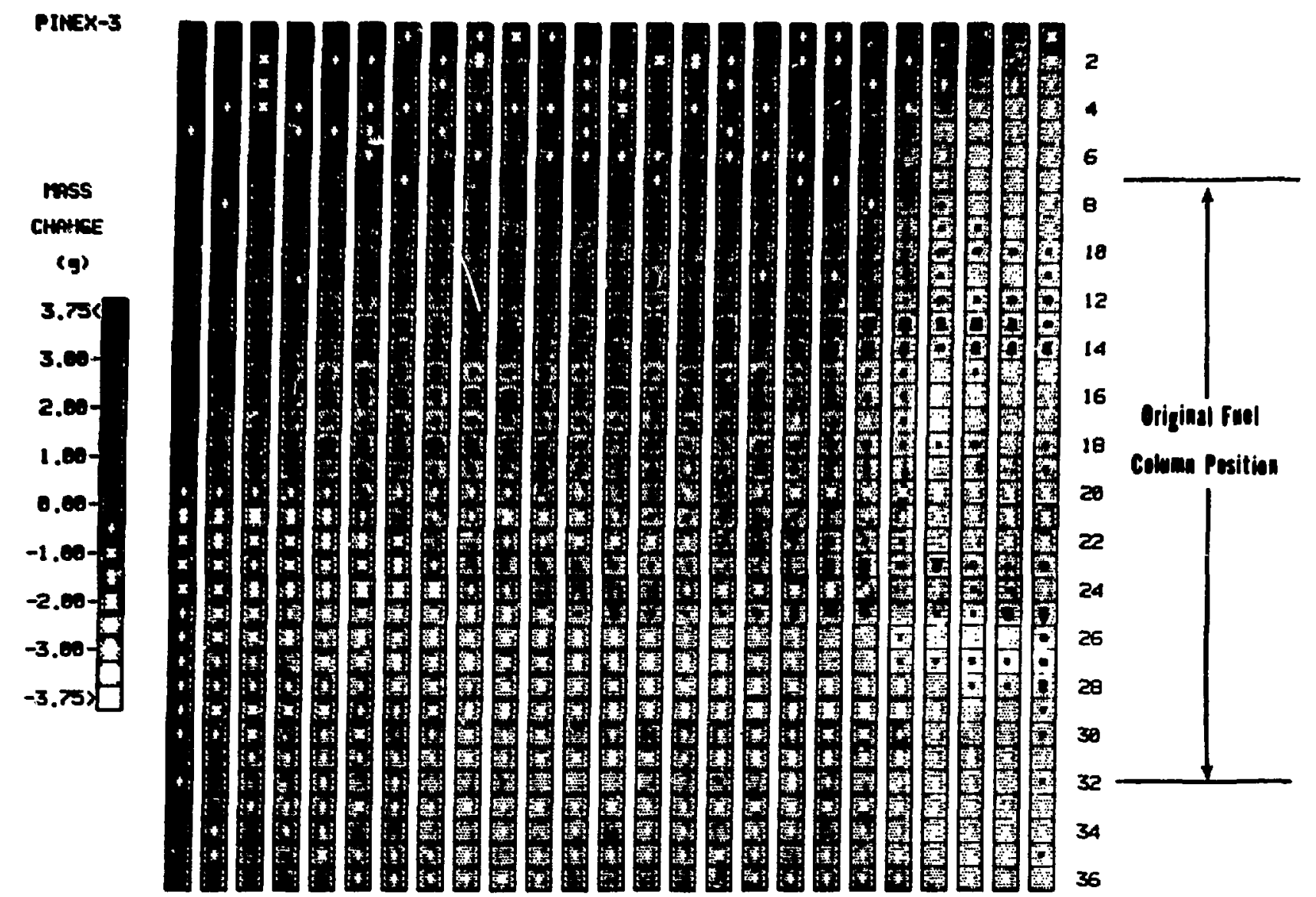

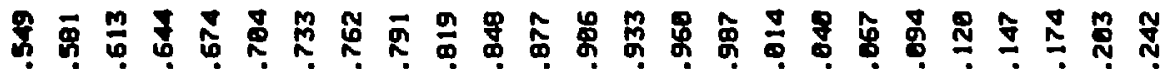

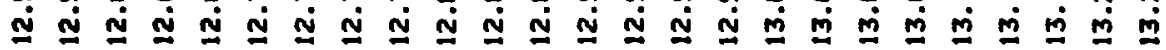

Fig. III-11. Axial component of fuel motion. Symbolic hodograph series depicting changes in net axial fuel disposition for a single-pin transient (PINEX-3). Because clad failure took place at $12.55 \mathrm{~s}$, the axial displacements primarily represent motion in the coolant channel external to the pin. By $11.95 \mathrm{~s}$ about $2 \mathrm{~g}$ of fuel appears to have been redistributed within the upper half of the fuel pin annulus. By $12.22 \mathrm{~s}$, enough fuel accumulated in a region extending from 10 to $43 \mathrm{~cm}$ above the centerline to correspond to a possible internal blockage. By $12.52 \mathrm{~s}$ a strong inference could be drawn that clad failure had nccurred about 6-8 cm above the midplane. From 12.52 to $12.76 \mathrm{~s}$, other fuel relocated from below to above the midplane. At $12.82 \mathrm{~s}$ a net downward motion of fuel began; this could have been fuel movement outside the breach, down along the coolant channel. 


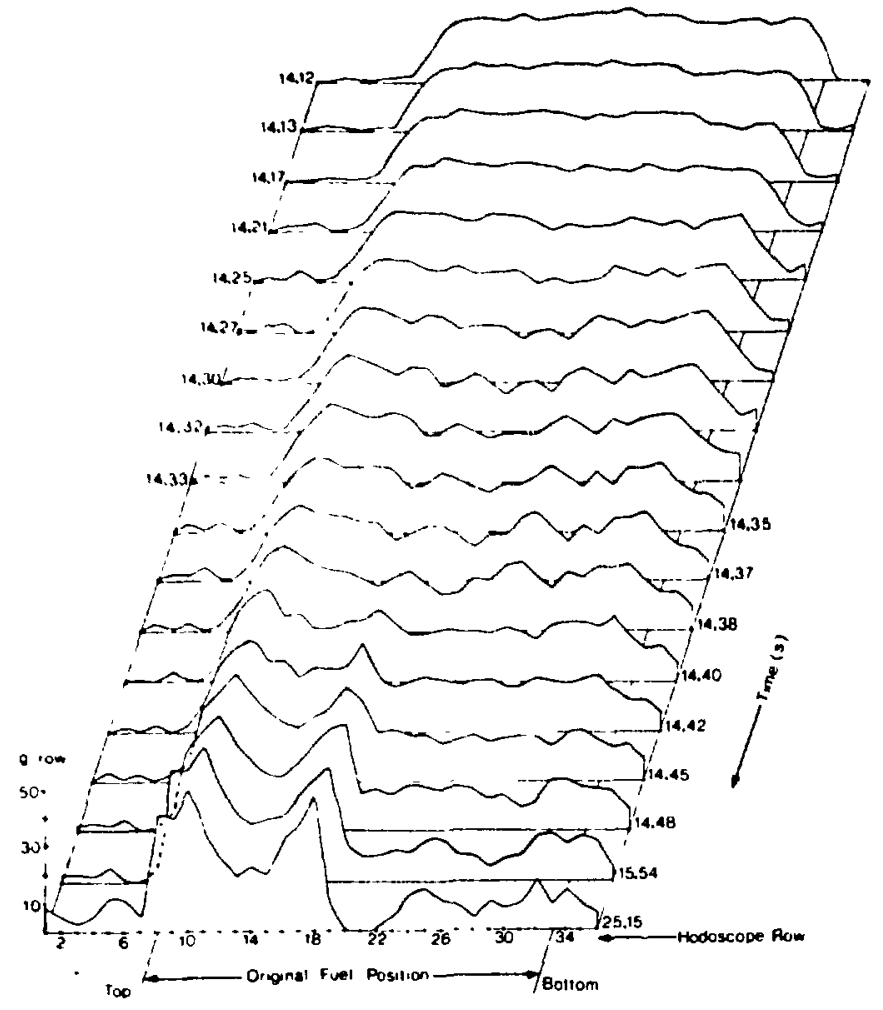

Fig. IlI-12. Mult iparameter plot of hodoscope data from TREAT transient L8. The abscissa is graduated into hodoscope rows, which run along the vertical axis of the three-pin fuel bundle. The amplitude of each profile is quantized in g/row. The combined axial profile of the fuel bundle early in the transient at $14.12 \mathrm{~s}$, which is prior to significant fuel motion, is shown at the top. The time-wise progression shows upward and downward relocation of fuel, eventually resulting in substantial voiding near the original midplane and the formation of two upper blockages. Post-test examination has confirmed the final disposition.

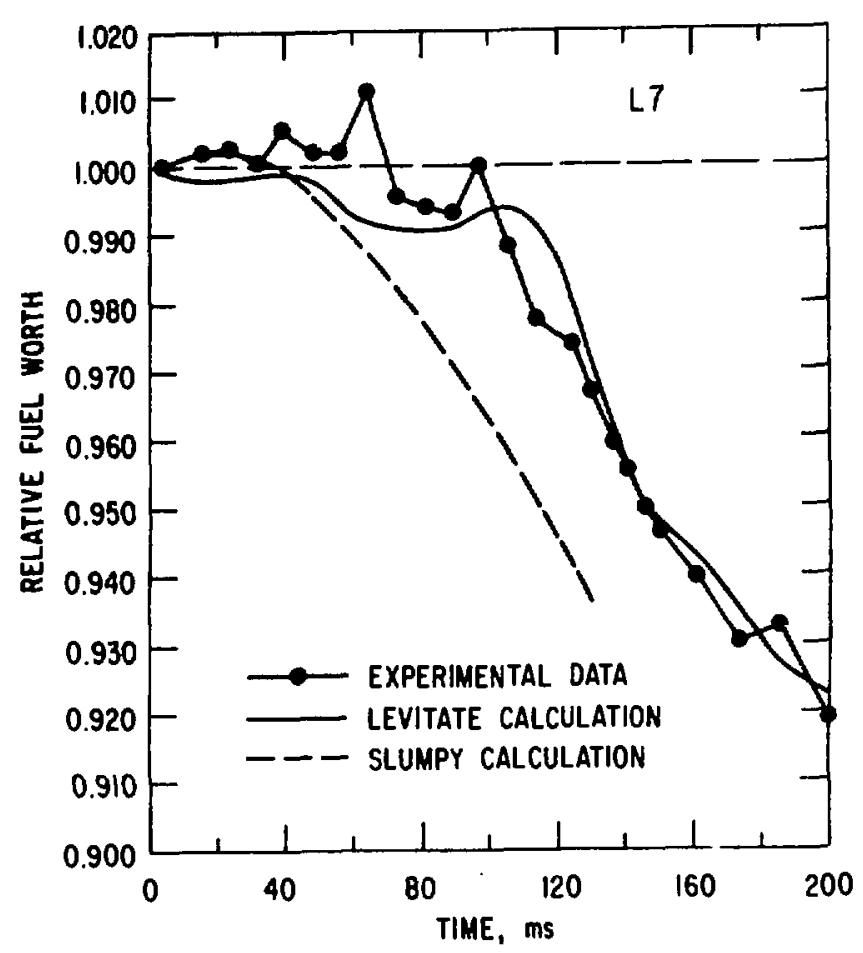

Fig. III-13. Worth-curve comparison. Hodoscope experimental data extrapolated to larger cores is compared with calculations from accident-analys is computer codes. SLUMPY is an early fuel-motion modeling code that does not have some of the advanced features introduced in LEVITATE. The results indicate that fuel movement tends to diminish the reactivity and therefore to induce a safe shutdown of the reactor. 
A wide range of fuel motion phenomena have been observed by the TREAT hodoscope, including pin bending, axial and radial expansion, prefailure motion (within cladding), internal fuel motion (within annular fuel), fuel breach into coolant channels, sweepout by coolant, eructation, slumping, blockages, breach of flow tube, and compaction.

Recent results [HE-82] for the CABRI hodoscope indicate the detailed radial and axial evolution of single-pin distortion and destruction. Experiment A4, which used a 8-mm zirconium vessel to provide a larger signal than the previous 4-mm steel container, resulted in rapid $(10 \mathrm{~m} / \mathrm{s})$ expulsion of fuel over a $15-\mathrm{cm}$ height. Fuel eventually collected at a lower spacer structure. In BI1, bending by about $2 \mathrm{~mm}$ was followed by expulsion of fuel into the coolant channel; an unusual effect observed was the growth of an interpellet gap. This was the first experiment in which the hodoscope was lined up with the center of the pin split evenly between two channels, thereby leaving one channel as a more independent background channel. Experiment $\mathrm{Al} 2$ led to central voiding of fuel and the detection of delayed neutrons from fission products collected at liquid sodium interfaces. These data have played an important role in analysis and application of the CABRI in-pile program [ME-82].
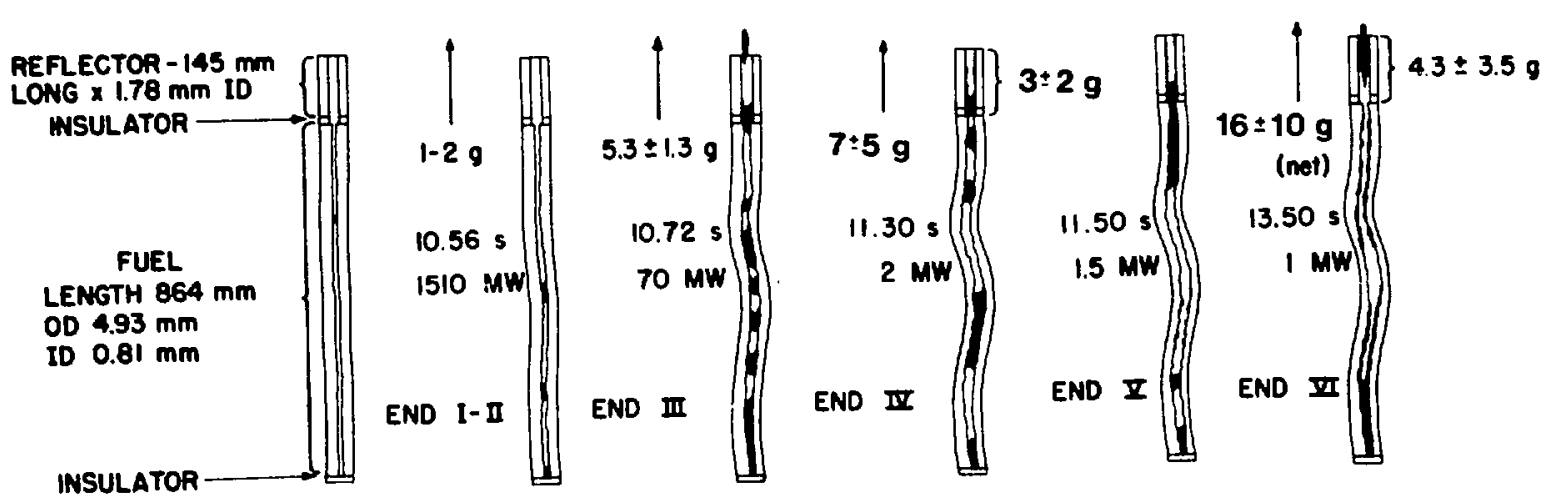

Fig. III-14. Artist's reconstruction of PINEX-2 fuel movement. The cladding did not fail during this experiment, and the axial fuel motion observed was confined to the central $0.8 \mathrm{~mm}$ cavity within the fuel pellets. Fuel in the amounts estimated was ejected into the reflector zone, and post-test examination was in good agreement with the final configuration at $13.50 \mathrm{~s}$. 


\section{ALTERNATIVE DIAGNOSTIC DEVICES}

Starting in 1974, evaluations of methods of fuel-motion diagnostics based on newer technology were made. Two U.S. laboratories undertook investigation and development of several alternatives, and a multinational project chose to build a hodoscope at Cadarache, France.

There were three overriding objectives for these re-evaluations. First, the fast-reactor safety program was moving towards additional safety facilities that would require fuel-motion diagnostic systems; second, much larger fuel bundles were scheduled to be tested; and third, it was desired to reduce some of the shortcomings of the existing TREAT hodoscope, particularly in terms of spatial resolution.

Three types of ex-core high-resolution diagnostic systems alternative to the hodoscope have reached an advanced degree of experimental scoping: flash $x$-ray, pinhole, and coded-aperture. The flash x-ray technique depends upon detection of changes in transmission of accelerator-produced bremsstrahlung through density variations in the test fuel. The others require that the test fuel emit detectable (gamma or neutron) radiation as a result of fission. In-core detectors, which depend upon preferential localized detection of fuel fission radiation, were also considered for coarseresolution applications.

\section{A. Flash radiography}

Two variations of the flash $x$-ray system have been proposed. Both depend on passing a beam of high energy $x$-rays (up to $20 \mathrm{MeV}$ ) through the test section; they require, accordingly, a full-slot through the test-reactor core.

The most straightforward scheme (Fig. IV-1) [ST-76a, ST-76b, $\mathrm{CH}-76$, ST-76, HA-76, P0-76] entails the projection of a beam of bremsstrahlung produced from electron interactions with a heavy-metal target. The PHERMEX facility [DI81] at Los Alamos has been used for development of this method, and single-shot well-resolved images have been produced for 217-pin fuel bundles in a thick steel container. To obtain the required resolution, either a narrow-focused beam must scan the large test bundle area, or a broad-area beam must be imaged by a position-sensitive detection system that would probably include a collimator and detector bank.

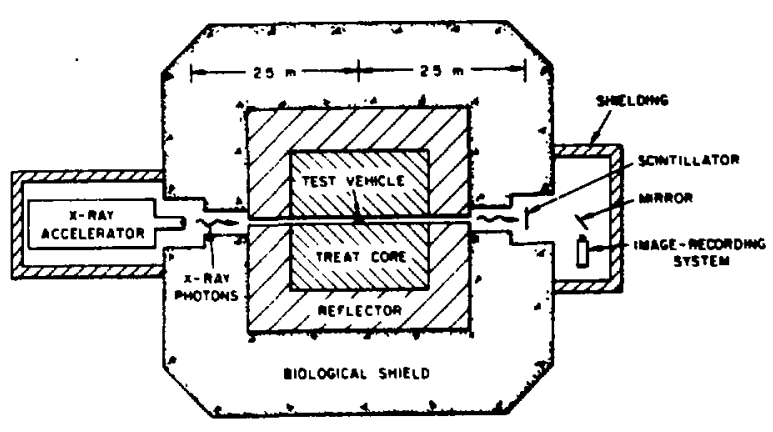

Fig. IV-1. Flash X-ray fuel-motion diagnostic method. High-energy $x$ rays are attenuated in their passage through the test vehicle, and changes in fuel density appear as changes in transmitted beam. Because the accelerator beam is a point source, the detector vertical dimension would have to be about 2.4 $m$, unless the accelerator is moved further away from the reactor, which would reduce its transmission intensity. 
A second technique [KE-76a, KE-76b] encodes a broad-area accelerator source before transmission through the test article. This should yield superior modulation, a wider field of view, and overcome the source broadening and poor resolution associated with narrow-beam sources. Either a coded source or coded beam may be used.

Sandia [ST-79] has estimated that an accelerator necessary to meet current TREAT diagnostic requirements would need to deliver $1 \mu \mathrm{s}, 5 \mathrm{~A}, 30 \mathrm{Mel}$ pulses continuously at the rate of $1000 / \mathrm{s}$. No machine with all these capabilities has been built, nor is any on the horizon likely to be reliable and inexpensive enough to be acceptable for in-pile fuel-motion diagnostics use.

\section{B. Coded aperture}

A passive coded-aperture system for emmitted gamma radiation [KE76c, KE-79, MC-76, ST-80] has been under development for over seven years at Sandia Laboratories. A modified Fresnel zone plate was originally used to modulate the radiation produced in the fuel pin at the Annular Core Research Reactor (ACRR) (Fig. IV-2); it has been replaced with a uniformly redundant array (URA) in order to improve resolution [KE-82]. The coded image or psuedohologram recorded at the detector plane is treated either optically or digitally to reconstruct the image of the source distribution. Because the fuel source is long and thin, a onedimensional linear aperture is used to produce the effect of pinhole imaging in the vertical direction.

The detector plane, the coded aperture, and the vertical pinslit aperture are respectively $1.37 \mathrm{~m}$, $0.78 \mathrm{~m}$, and $0.92 \mathrm{~m}$ from the fuel plane. In order to image a fissiongamma-ray spectrum ranging up to 8 $\mathrm{MeV}$, zone thicknesses are nearly 1.5 $\mathrm{cm}$ with widths of outside zones kept below $0.5 \mathrm{~mm}$. A gold-plat inum alloy is used for the zone plate, and Kennertium for the vertical aperture. Typical attenuation coefficients are about $1.3 \mathrm{~cm}^{-1}$ for 1-MeV gamma rays.

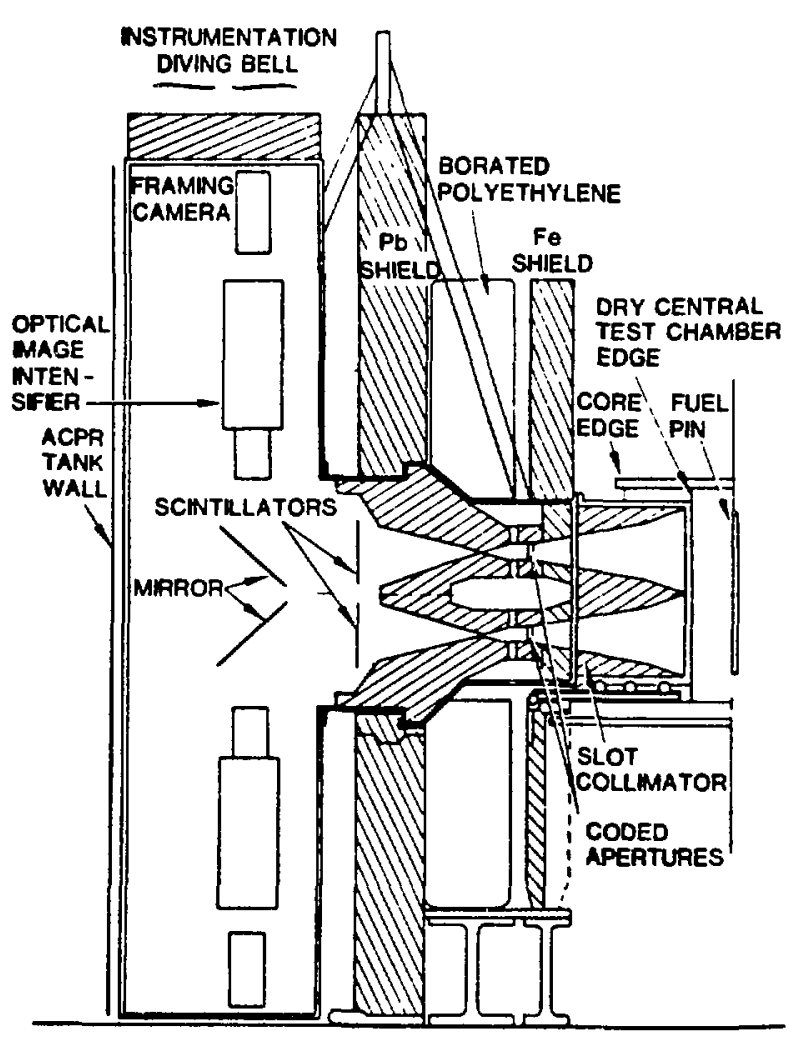

CAIS/ACRR

Fig. IV-2. Coded-aperture diagnostic instrument system (CAIS) at the Sandia ACRR. The entire apparatus is enclosed in a diving bell that can be inserted or withdrawn from the water reflector region of the reactor. 
The electro-optical imaging system consists of a $0.76 \mathrm{~mm}$ thick calciumtungstate scintillator coupled through a rotating mirror to a high-gain image intensifier. The image is photographed with a $16-\mathrm{mm}$ pin-registered camera at up to $1000 \mathrm{frames} / \mathrm{s}$.

In order to maintain the largest possible viewing angle and to cope with space limitations, the aperture and electro-optical system are contained in a chamber that is lowered to the bottom of the react or water tank. Massive shielding and collimation to exclude extraneous background are critical to performance.

\section{Pinhole imaging}

A pinhole system has been developed at Los Alamos National Laboratory for application to TREAT [BE-76b, BE-79, LU-79]. The design was based on national-security-classified results of successful pinhole application for imaging nuclear-weapons explosions during underground testing. Since then, extensive testing, which is summarized in Sect. $V$, has taken place under appropriate reactor conditions.

As shown in schematically in Fig. IV -3 , the source is imaged by a pinslit. The tapered slit, made of Kennertium, brass, and polyethylene, has an aperture tailored for the experiment. The smallest pinhole had horizontal and vertical diamensions of 0.4 and $3.0 \mathrm{~mm}$, and it is $1-m$ thick at its largest dimension. With the source and detector each located about the same distance from the aperture $(2.6 \mathrm{~m})$, the limiting geometrical resolution is about double the pinhole dimensions. In order to cover the vericical field of view for typical TREAT tests, four pinhole units were stacked, and each had independent image-recording systems.

The image-recording systems consisted of a scintillator and a high-speed intensification camera system, coupled via a mirror. An earlier system also intensified the light beam and exposed a high-speed framing camera. Video images from the vidicon may be produced in 256line analog form every 8 or 16 ms. The NE102 plastic scintillator has a relatively high sensitivity to gamma rays.

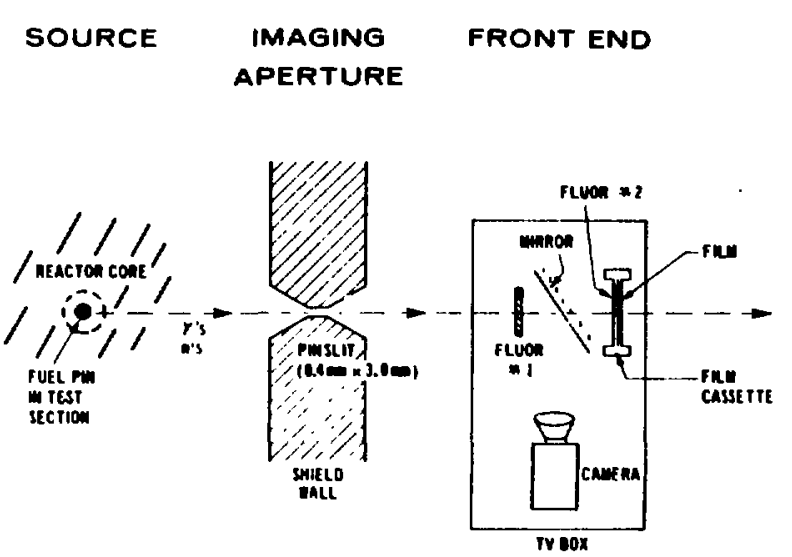

PINHOLE / TREAT

Fig. IV-3. Schematic arrangement of a pinhole-camera diagnostic imaging device tested at TREAT. The pinslit is mounted in the reactor biological shield, making use of the slot between the test section and the aperture. Extensive shielding surrounds the instrumentation box. The film cassette has been used for time-integrating images of stationary fuel. 
The vidicon is the limiting instrument in dynamic response range. With a $\mathrm{Sb}_{2} \mathrm{~S}_{3}$ target the linear range above dark current for a typical tube [NO-80] is 3.3 and the usuable range is no more than a factor of 20 up to saturation. An increase in power by a factor of 8 would yield a response $20 \%$ nonlinear compared to a setting equal to the dark current. If signal strengths somewhat below dark current can be accurately derived, the upper limit in dynamic range for the pinhole system would be no more than about 100 .

Data can be converted after an experiment to digital form so that digital processing techniques could be applied for reconstruction. The $256 \times 512$ pixel arrays for each TV frame are binned into $0.6 \mathrm{~mm}$ by $6 \mathrm{~mm}$ pixel resolution in terms of the source plane. Digital-data fitting procedures, field-of-view efficiency corrections, power normalization, and background subtraction can then be carried out.

Under optimal statistical conditions (sufficient time integration during steady-state imaging), the pinhole can achieve single-pin resolution sufficient to distinguish the $1.5 \mathrm{~mm}$ central void with about $0.8 \mathrm{~mm}$ resolution.

A coded array of pinhole detectors in a non-redundant zone configuration [BE-76a, HAN-76a, HAN-76b, HAN-76c] was tested at TREAT too. The 20 pinholes were distributed so that the vector distance between any two occurs only once. Data was collected on an imaging system similar to that employed for the single pinhole systems. Analog data can be reconstructed by coherent or incoherent optical systems.

\section{In-core detectors}

Miniature detectors may be placed in-core for local coupling to neutron or gamma radiation emanating from the fuel. In fact, they may be placed within the test section. Sandia National Laboratory has developed fission couples for such use [DU-76, ED-76, WR-76, WR-79] and Argonne National Laboratory devised a string of partially directional fission counters that was tested in experiments at ETR [COX-76a, COX-76b]. In-core detector ratios of $235 \mathrm{~J} / 23 \mathrm{U}$
have been measured in a $37-\mathrm{pin}$ subassembly at PARKA [EV-79b]. 


\section{COMPARISON OF DIAGNOSTIC CAPABILITIES}

In evaluating comparative performance, the following attributes are objective indicators of fuel-motion diagnostic-system quality: signal/background ratio, sensitivity and statistics, spatial resolution, time resolution, mass resolution, and dynamic range. Statistical interdependence of these attributes makes it necessary to standardize the basis for any comparison.

The signal/background (S/B) ratio is important for development and intercomparison of instrument systems. When instrument noise is relatively large compared to signal strength, the signal/noise ratio is usually measured. Because of the dominating effect of the radiation background incident on the detector in a reactor environment, it is inadequate to compare signal without taking into account both instrument noise and scene background effects.

Detection sensitivity $(\Delta S)$ depends upon signal/background ratio, signal (fuel mass) change, power level, daca-collection interval, and method of background nomalization [DEV-82a]. A comparative estimate of sensitivity in terms of signal strength (energy deposition in the fuel, $\mathrm{J} / \mathrm{g}$ ) can be derived from evaluation of the threshold for detection of a known amount of fuel at a common level of confidence, usually one standard deviation $( \pm \sigma)$.

Spatial resolution is largely determined by geometrical parameters of the imaging system and limited in practice by noise and artifacts. In fuelmotion diagnostics, it is not necessary for horizontal $(\Delta x)$ and vertical $(\Delta y)$ spatial resolution to be identical with each other (nor constant in the field of view); as long as they are adequately characterized.

Because of its statistical dependence, time resolution $(\Delta t)$ cannot be expressed simply in terms of data-collection intervals. The limiting timeresolving factor for a given experiment is often not the instrument capability but the statistical quality of the data in terms of reactor power level and specific fuel-motion events.

Inasmuch as reactivity effects are most closely associated with fuel movement, quantitative mass resolution $(\Delta \mathrm{m})$ is perhaps the most significant and telling parameter for fuel-motion diagnostics, given that a derived resolution can be achieved over a wide enough range of conditions.

Dynamic range $\left(P_{\max } / P_{\min }\right)$ is an important functionai parameter due to the wide power range of a transient reactor during an experiment and the differing power shapes and amplitudes experienced in going from one experiment to another.

Several subjective factors are also important, such as operability of the system, recordability of data, susceptibility to artifacts, and convertibility to useful processing format. These factors are treated at the end of this Section. 


\section{A. Theoreticai considerations}

Mass resolution depends largely upon power level and upon integration of data-collection time and spatial domains. Mass and/or spatial resolution can also depend upon the geometrical form of the fuel. For example, a segmented system like the hodoscope can sense very small deflections or translations of rigid fuel, but it has much poorer resolution (equivalent to the interchannel spacing) for entirely incoherent mass changes. On the other hand, for diagnostic systems or data that have low signal/ background ratios, model-dependent reconstruction algorithms are often invoked, making practical resolution limits dependent upon the geometric as sumptions.

A change in response due to transverse movement of the fuel center of mass can be estimated within statistical limitations to the extent that the response function of any single detector or pixel is known. This is illustrated in Fig. $V-1$. The spatial resolution of segmented (hodoscope) systems is not necessarily limited by interchannel spacing. Detection in an adjacent channel provides the elements of a time-dependent vector which can localize the direction, time, and coordinates of the center of mass. Realizable resolution limits, substantiated by considerable data, have been demonstrated in practice to be far better than the interchannel spacing for such highly correlated fuel motion as radial expansion.

Although Fig. V-1 applies directly to hodoscopes, it also corresponds to position-sensitive imaging systems if data over an equivalent number of pixels is integrated, as usually done to achieve adequate statistical definition.

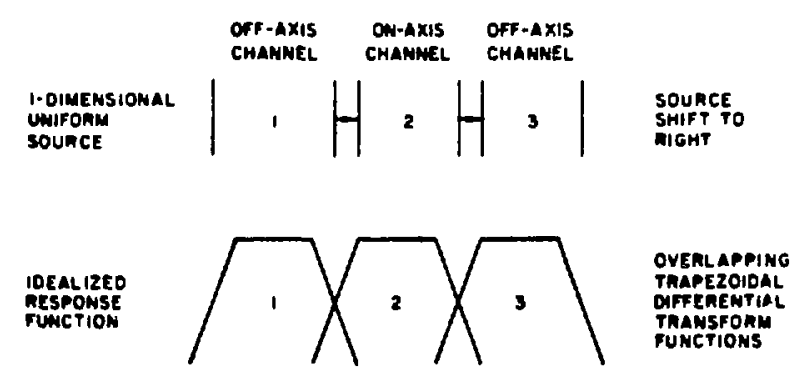

(0)

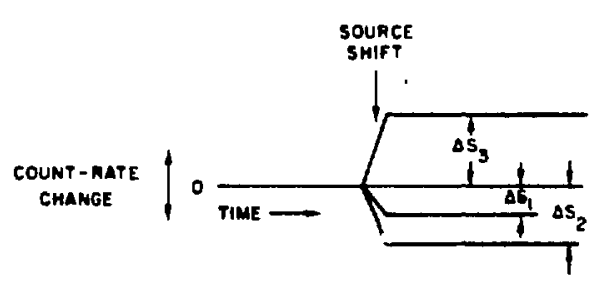

(b)

Fig. V-1. Simplified model of response functions. A 3-channel model, based on a 1-dimensional uniform source, demonstrates how a lateral translation of the source uniquely affects the count rate of all three channels. A confined source with dimensions smaller than the flat-response portion of the idealized overlapping trapezoidal transform function would be the only case that would not propagate a change in count rate. In the actual 2-dimensional situation, the likelihood is small (a few percent) of a small clump of fuel remaining in undetected motion (orbitting) within the vertical and horizontal transforms. 
In actual single-pin experiments, much of the fuel motion that takes place is correlated between channels as a result of various physical constraints, such as the initial rigidity of the fuel, the restraint of cladding, and the axial channeling effect of a voided central annulus. Because of contiguity, center-of-mass displacement may be accurately interpreted with little risk of ambivalence from alternative explanations. In fact, the probability of fuel motion remaining within single-pixel orbits, thereby being undetectable, can be calculated from geometrical considerations. The probability of motion that lacks center-of-mass displacement is only a few percent for the present hodoscope design, and such movement without net reactivity effects is of no real interest to the reactor-safety program.

High precision for these results from the hodoscope is due to several factors. Foremost is the choice of a system design that, considering the role of statistical and systematic effects, maintians a relatively high signal-to-background ratio. Another major contributing factor is that the response of a single hodoscope channel is unique t.o all but a few possible modes of fuel motion. Then, with overlapping resfonse functions, advantage may be taken of correlations between channels to further minimize ambiguity. In practice, a multi-channel flat-response integral avoids the necessity for deconvolution. These considerations can easily be verified experimentally by taking advantage of an equivalence axiom: fuel movement across the detector projection (pixel) is equivalent to scanning the pixel across stationary fuel; it is relative motion that is detected by the hodoscope. As a result the detailed resolution achieved in Fig. III-6 is indicative of the motion resolution that can be achieved in a transient, all statistical and systematic factors being the same.

Another favorable factor that may be used to improve sensitivity of diagnostic systems is the continuous and extensive monitoring of background to acnieve high statistical precision and systematic accuracy. Fuel-motion detection can take place at a threshold much lower than pin "visibility," which is a static condition.

\section{Signal-response for fuel expansion and clad breach}

Resolution limitations apply to all diagnostic systems, because, in practice, data must be integrated over object pixels in order to achieve adequate statistical value over some or all of a transient. A single hodoscope channel may be compared against a similar-sized pixel of other imaging systems.

In order tiv evaluate performance under standard condition, particular scenarios ma, be defined. Some that arise in practice are pin expansion, internal fukl motion, and clad breach. These have in common certain characterizing geometrical constraints.

In Fig. $V-1$, note that at a single elevation, (radial) pin expansion would create significant signal changes in both of the on-axis channels. This response is distinguishable from other phenomena, such as pin expansion versus clad breach, which differ in signature: pin radial expansion is relatively uniform along an axial string of pixels, whereas breach is a localized event that propagates radially and/or axially in time. 
Consider two alternative models for interpreting pin expansion. In one model the inner (hollow) radius of the pin is held constant and the outer radius expands; for the other model, both inner and outer radii expand, kecping constant fuel density. For $70 \%$ pin expansion, the two models are theoretically distinguishable on the basis of differences in the signal reponse. The expanding inner radius results in $15 \%$ gains in of f-axis rates and $33 \%$ losses in on-axis rates; the fixed inner radius has $9 \%$ gains off-axis and $20 \%$ loss on-axis. However, the second model with expansion to $8.94 \mathrm{~mm}$ is not distinguishable from an expansion to $\sim 7.4 \mathrm{~mm}$ with $\sim 5.2 \mathrm{~mm}$ ID in the first model.

Other cases may be found in which ambiguity in the result may be postulated. Sometimes the ambiguity can be removed by calibration or better characterization of the model. For example, in the case of pin-expansion model ambiguity, post-test examination data could reveal whether the inner diameter remained fixed or expanded. In any case, the practical feasibility of distinguishing between models depends strongly on statistical criteria.

Three general characteristics of fuel-breach differ from the signal response due to pin expansion: horizontal asymmetry (unidirectional movement), off-channel signal amplitude ( $17 \%$ in this example), and vertical range of initial effect (localized to a single elevation). In practice, cladding breach is also accompanied by two other features. First, there is no comparable local loss because fuel is usually emerging from within a selfshielded fuel pin. Second, and very important, is a disproportionate enhancement of signal brightness to the many-fold increases in power coupling for small amounts of fuel that have escaped into the coolant channel.

The absence of a signal loss in the middle channel during cladding breach is consistent with experience that fuel which pours through the breach usually emanates from internal melting distributed along the central axis of the pin rather than uniqualy from the pellet at the breach. If the fuel originated at the breached pellet, some losses in the middle column should appear -- though masked by the self-shielding of incident neutrons.

Although the theoretical limitations of these models appear to be appreciated, the practicai constraints have not been as well identified, resulting in achievement falling short of prediction in situations where the diagnostic instrument cannot fulfill the stochastic or systematic conditions. In cases where the conditions have been met, nearly unambiguous sensitivity to phenomena has been the rule rather than the exception.

\section{Detection sensitivity and figure of merit}

Beca.'se any particular experimental situation in fuel-motion diagnostics entails sensitivity to changes from original fuel distributions, under widely varying power ievels, statistical considerations require some particular attention. The parametric dependence of detection sensitivity on signal/ background ratio, along with a figure of merit, has been derived [DEV-82a] (see Fig. V-2). A simplified expression that normaiizes background based on an early interval for estimating initial count rate $\left(s_{0}\right)$ dependence on initial signal/background $\left(s_{0} / b_{0}\right)$ for a given fractional mass loss $(\delta)$ is 
$s_{0} t \delta^{2}=1-\delta+b_{0} / s_{0}$.

The figure of merit obtained is

$$
\begin{aligned}
F & =\left[\left(s_{0}\right)_{2} /\left(s_{0}\right)_{1}\right]^{1 / 2} \\
& \times\left[\left[1-\delta+\left(b_{0} / s_{0}\right)_{1}\right] /\left[1-\delta+\left(b_{0} / s_{0}\right)_{2}\right]\right]^{1 / 2} .
\end{aligned}
$$

$F$ values are not especially meaningful until they become as large as three. Actual achievement of the maximum capability in precision aid accuracy depends on correct subtraction of background and on wide dynamic range in count rates. Hodoscopes have relatively high signal/background compared to other imaging systems. Whether small or large fuel movement is being observed, $F$ can be equal for both types of systems if imaging systems have count rates about ten times larger than hodoscopes. All things being equal, a system with high signal/background is preferable from the viewpoint of avoiding problems from artifacts in recording and reconstruction.

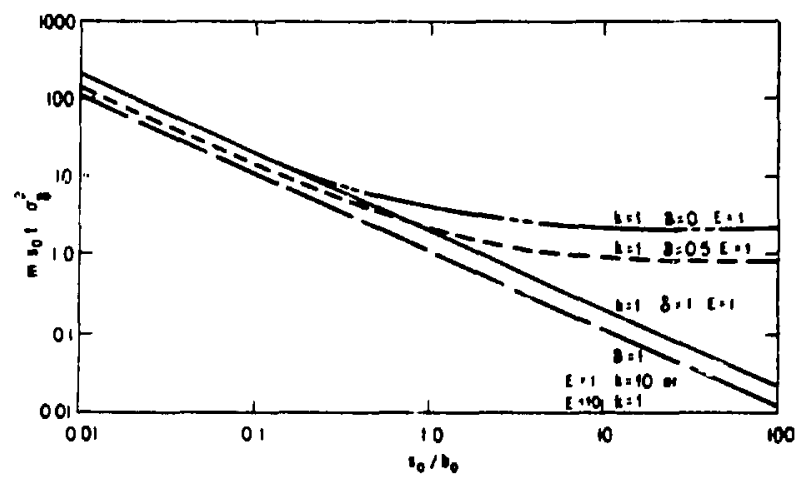

Fig. V-2. Signal/background functional relationships. Relative detection sensitivity $s_{0} t$ for a given standard deviation $\sigma_{\hat{\delta}}$, depends on initial signal/background ratio $\mathrm{s}_{\mathrm{o}} / \mathrm{b}_{\mathrm{O}}$, relative power level $\mathrm{E}$, relative number $m$ of time intervals $t$ and channels $k$ used for background measurement, and fractional amount $\delta$ of displaced fuel. Only a few counts are needed at high $\mathrm{s}_{0} / \mathrm{b}_{0}$ to detect a signifiant change in fuel disposition at a $68 \%$ confidence leve 1 .

\section{Image-restoration techniques}

In order to improve visual perception of images, restoration techniques are employed. These techniques are especially critical in systems of low signal/background, such as coded-aperture and pinhole devices, where the image would be otherwise swamped by background and noise. In general, such techniques have not been applied to hodoscope data, but investigations [RH82a; RH-82b] have been carried out for the purpose of improving spatial resolution when necessary.

Studies that have been made [RH-79, BAR-76] have largely been for internal dacision making. Experimental data is available in the next part of this Section.

\section{B. Direct comparison with pinhole systems}

Pinho'e-camera systems reached an advanced stage of development in application at TREAT, resulting in six direct comparative tests with the hodoscope -- PINEX-2, PINEX-3, C01, CO2, CO3, and AX-1. In single-pin test PINEX-2 some quantitative data on fuel motion external to the pin was observed by the pinhole, but it did not detect [BE-79] the internal fuel-pin motion that was measured by the hodoscope [RH-80]. 


\section{Experimental data for PINEX-3}

The most definitive experiment was performed with both the pinhole imaging system and the hodoscope operating simultaneously was PINEX-3. The pinhole system demonstrated significant progress [LU-80, LU-81] with increased sensitivity at lower power levels. A data exchange at a crommon date two months after the experiment was arranged between the experimenter and the two laboratories that operated the diagnostic systems.

Numerous detailed events, measured in terms of specific time, location, and mass were derived from hodoscope data, [MA-80] including information on latera: fuel motion. The level of hodoscope sensitivity during peak power was on the order of $0 . \hat{\mathrm{g}} \mathrm{g} / \mathrm{channel}$ for $\mathrm{i} 2$-ms integration.

Both diagnostic systems reported data regarding the time and location of fuel breach. The tine estimated for clad failure from thermohydraulic data coincides reasonably well with the hodoscope-reported time for "clad breach" which is keyed to radial fuel movenient, and to the pinhole datum on "clad failure." There were discrepancies in identification of the elevation of the breach and the location of subsequent fuel motion, discrepancies reriaining largely unresolved by results in the final pinhole report [LU-31].

The pinhole interpretation of cladding failure is based on pin-width measurements determined by digital analysis from TV-line scans; they describe the phenomenon as "pin expansion," whereas the hodoscope points more specifically to localized failure. Influencing the pin-expansion interpretation is the gamma-ray response of the pinhole scintillation detector. Estimates of the pinhole gamma-ray fraction have varied from 50 to $75 \%$. Consequently, the steel cladding which emits capture gamma rays can have a disproportionaie effect, particularly at the edges of the test pin. Swelling of the clad could account for the appearance of fuel expansion. While it may precede or coincide with clad breach, expansion is not the same phenomenon, and it is the failure time and location that is of most interest.

Apparent elongation of the fuel pin wus a feature jointly reported for each diagnostic device. The measured elonçation slopes were similar, but a 1-s disparity was present in time of onset of elongation.

The most significant feature noted unilaterally by the pinhole analysts was axial fuel expulsion into the reflector -- beginning at $10.8 \mathrm{~s}$, long before fuel solidus was reached. Pinhole data interpreted immediately after the experiment implied thit a significant amount of fuel was ejected upward prior to cladding failure; they "logged eight possible cases for fuel in the reflector," one case representing an estimated $4 \pm 3$ g ejected into the reflector. This was subsequently revised downward to $1 \pm 0.5 \mathrm{~g}$ ejected fuel.

No such fuel ejection was noted in PINEX-3 data by other instruments; the hodoscope did not see or report anything of that magnitude above its sensitivity level of $0.2 \mathrm{~g}$ or less. Neither the $x$-ray or thermal-neutron radiographs show significant fuel ejection. Only about $14 \mathrm{mg}$ of fuel was found above the insulator pellets in the post-test examination; no residual evidence of upward motion followed by draining was found on the inside surface of the reflector. 
Hodoscope data (Fig. III-11) after integration to $12.67 \mathrm{~s}$, shows about $8 \mathrm{~g}$ net fuel having moved upward -. though not expelled through the reflector. All mass estimates are uncorrected for self-shielding, which could increase the estimated magnitude of fuel displacement by $50 \%$ or more. The pinhole data give nonspecific "contrary" results.

In the conventional radiographs taken after the experiment, the annulus was still found to be plugged about $15 \mathrm{~cm}$ below the top of the fuel $(\sim 28 \mathrm{~cm}$ above centerline) [DEV-81b]; the hodoscope data is consistent with a fuel accumulation in this region at the end of the transient (last hodograph at $13.24 \mathrm{~s}$ in Fig. III-11).

In surimary, the hodoscope report [MA-80] included details of internal fuel motion (within the central $0.8 \mathrm{~mm}$ hole of the fuel pin), which was not observed by the pinhole; both systems reported certain features in common, including approximate agreement in time of cladding breach; and pinhole data spuriously indicated axial fuel expulsion during an early part of the transient.

\section{System quality indicators}

Signal/background ratio. From the available data [LU-82] the peak S/B ratio for pinhole transient imaging of PINEX-3 was $0.2 \pm 0.1$, an order of magnitude smaller than the hodoscope. A value of $0.4+0.1$ is achievea from film integration [LUM-80] of pinhole data. In the definition used here, all nonsignal sources (scattering and instrumental) as well as instrumental noise are included in the background.

Sensitivity and statistics. For the hodoscope its minimum threshold for "pin visibility" in PINEX-3 experiment was about $0.02 \mathrm{~J} / \mathrm{g}$ (corresponding to one pixel) [DEV-81b]. This measure of sensitivity is based on an observed signal/background ratio of 1.9 and a signal countrate of $10 \mathrm{cps}$ at $80 \mathrm{~kW}$.

For the pinhole, an equivalent pin sensitivity threshold can be estimated to be a 16-ms frame (or "field") of data recorded with a power level of about $300 \mathrm{MW} \mathrm{--}$ or $5 \mathrm{~J} / \mathrm{g}$. A subjective determination of a lower bound for power level required for pin "visibility" would be $110 \mathrm{MW}$ at $16 \mathrm{~ms}$, or $1.8 \mathrm{~J} / \mathrm{g}$. From the noise level in the 32-frame average, sensitivity would appear to be about $6 \mathrm{~J} / \mathrm{g}$.

The preceding estimates are based on statistical considerations, and as such are more objective than visual comparisons of pictures. Taking the pinhole sensitivity at a lower bound of $2 \mathrm{~J} / \mathrm{g}$, the hodoscope can sense equivalent fuel-motion at power levels a factor of 100 lower than the pinhole.

Vertical and horizontal resolution. Along the fissile length of the pin, pinhole horizontal resolution was $0.8 \mathrm{~mm}$, and in the reflector/plenum region above and below the fissile fuel it was $1.6 \mathrm{~mm}$. The larger pinhole yields higher count rates. 
The hodoscope was able to distinguish axial and radial fuel movements with better apparent resolution than the pinhole. Vertical resolution for both the pinhole and hodoscope appears to be about the same (about $6 \mathrm{~mm}$ ) in the central zone.

Although better pinhole aperture resolution could be designed into the system, marginal performance makes impractical such tradeoffs. In fact, improvements were made in pinhole sensitivity for the PINEX-3 test, partly by sacrificing spatial resolution in order to have a larger radiation acceptance aperture, and partly by use of sophisticated digitization and reconstruction techniques [LU-79].

Time resolution. In the PINEX-3 experiment, time resolution requested by the experimenter was 6 to $12 \mathrm{~ms}$. The data-recording intervals for the hodoscope were $2 \mathrm{~ms}$, but some of the analysis was done with 6-ms accumulations and the remainder was based on 12-ms averages. The pinholeimaging device recorded data in $16-\mathrm{ms}$ intervals, and a number of such frames were usually averaged to identify discrete events.

From the time period 12.55 to $12.95 \mathrm{~s}$, with power level in the order of $100 \mathrm{MW}$, the pinhole data was averaged for about $0.5 \mathrm{~s}$; hodographs averaged over $30 \mathrm{~ms}$ during the same period contain minimal statistical fluctuations. other quantitative data reported by the pinhole is based on 80 -ms intervals between data points during the higher power phases of the experiment. Although 16-ms pinhole resolution is given for several parameters as a function of time, the data were integrated over a vertical dimension of $6 \mathrm{~cm}$ [LUM-82].

Mass resolution. Hodoscope mass resolution initially reported for PINEX3 was at the level of $200 \mathrm{mg}$ or better at the time resolutions given, which corresponds to typical hodoscope experience with capsules of this type under the given irradiation conditions. This result appears to be five or ten times better than the pinhole, for which no explicit values are supplied by the pinhole analysts. The hodoscope was able to detect and graphically portray the specific course of transient fuel events, giving differential data on the masses of discrete segments of fuel, information that was largely unreported for the pinhole. With additional time-averaging, an apparent hodoscope threshold of about $50 \mathrm{mg}$ was achieved in order to compare results with a calculated pre-failure axial profile.

Pre-failure fuel motion through the central annulus is important in understanding fuel ejection mechanisms and reactivity effects. If the $0.8 \mathrm{~mm}$ central cavity of the PINEX-3 fuel pin were filled, it would hold in one hodoscope channel height $(3.4 \mathrm{~cm})$ about $0.2 \mathrm{~g}$ of fuel, which is near the sensitivity limit of the hodoscope in this type of experiment $(0.1$ to $0.2 \mathrm{~g})$. The actual signal from that fuel would be less than $0.2 \mathrm{~g}$ equivalent because of flux shielding by the outer annulus. As a result, the ability to sense pre-failure and post-failure internal fuel motion is an observable threshold for an objective comparison of sensitivity.

Complete evolutions of axial fuel motion inside the pin for PINEX-2 [BE79] and PINEX-3 experiments have been reported by the hodoscope (e.g. Fig. V$3)$, the results being consistent with post-test examinations. In neither experiment has any internal fuel-motion data been reported for the pinhole 
apparatus. Fuel-motion results that have been reported for the pinhole device have only been for integral data or motion outside the pin.

Integrated data underestimates the magnitude of fuel motion because movement in opposite directions tends to cancel and unidirectional movement can be diluted. Signals for fuel motion outside the pin are enhanced by the iack of self-shielding along the fuel pin and a reduction in axial-filter thickness in the upper reflector region. Consequently, differential data is needed for these single-pin experiments.

Dynamic range. The dynamic range of the hodoscope is nominally a factor of 10,000; that is, useful data can be (and is) obtained in a single transient where the power ranges from 1 to 10,000 MW. The pinhole imaging system is limited by its vidicon to a dynamic range of about 100 [NO-80]. At the high end of the vidicon scale, signal intensity must not exceed the range of saturation, or blooming. The narrow dynamic range affects not only the data range for any transient, but it also necessitates a transient calibration procedure for the pinhole recording system that must be undertaken before each experiment. This calibration "tunes" it so as to center response within its dynamic range, avoiding loss of data from over or underexposure. No comparable calibration is required or conducted for the hodoscope.

\section{Performance factors}

Other diagnostic performance factors, though more subjective, are relevant in evaluation of system performance.

Discontinuities in data. All instrument systems have data impacted by both stochastic and spurious events, and their accommodation is a measure of inherent and applied system success. A careful inspection [DEV-81b] reveals that pinhole system data is not immune from discontinuities and nonuniformities that require compensation and unpredictable corrections.

Two types of visual discontinuities are present in the pinhole data. With four pinholes needed to view the $(1.12 \mathrm{~m})$ vertical field, the images from overlapping fields of the four pinholes must be joined at three junctions. The flyback trace (diagonal black lines) of the vidicon adds an additional discontinuity in one field. In addition, there are nonuniformities that, without the aid of other infornation, cannot be identified as effects indicative of real or phantom fuel motion.

The TREAT hodoscope also has discontinuities (interchannel gaps due to collimator design), but these are uniformly distributed and commensurate. The gaps are not inherent, representing a design compromise for the TREAT system; the CABRI hodoscope was designed without discontinuities in response function.

In hodoscopes with their large detector arrays, occasionally some detectors will fail or behave irregularly during a transient. These defects are usually smoothed over by auto- or cross-correlation. 
Nonlinearity. The Hornyak-button hodoscope detectors have been known to exhibit a supralinaar response that was particularly noticeable at high TREAT powers [DEV-82a]. Various measures have been taken to ameliorate this condition, including the use of lead $(\mathrm{Pb})$ filters. Software has been developed to provide analytical corrections. A new detection system using proportional counters is now instal led at TREAT to operate in tandem with the Hornyak buttons, and the proportional counters have not displayed supralinear effects in tests [FI-80]. The design is based on proportional counters developed for the CABRI hodoscope [BL-76].

A time-dependent axially asymmetric "flux anomaly" is proposed in the final pinhole report to account for some effects in their data during the interval between 12 and $13 \mathrm{~s}$. The anomaly is evidently a manifestation of nonlinear pinhole instrument behaviour [DEV-82e]. The phenomenon is not observabie in any of the simultaneous hodoscope data. The maximum values have two patterns distinctly coinciding with separate imageconverter/intensifiers; moreover, the effects appear to be concentrated in the two of four converter cameras that see the highest flux.

Artifact susceptibility. Inasmuch as the PINEX-3 experiment was designed to evaluate fuel ejection from internal fuel motion, good precision and accuracy of fuel-expulsion data is a foremost requirement. The eight cases in pinhole data that "may be attributed to ejected fuel" are more likely due to pinhole imaging artifacts. No axial fuel ejection was observed by the hodoscope; yet pinhole case 3 , originally reported to be above $4 \mathrm{~g}$, is an order of magnitude greater than the hodoscope level of sensitivity for a null event. Case 3 alone, aside from their seven other events, would represent a significant amount of fuel that would have been discernible in the $x$ radiographs and post-test examination, neither of which show any significant fuel in the plenum or reflector. (Of many transients examined, none have resulted in ejection without residual evidence of ejecta). In addition, pinhole data has not shown fuel losses elsewhere in the original pin to compensate for the ejected fuel.

Because the pinhole apparatus has a low signal-to-background ratio, it is subject to misleading artifacts that might remain after background subtraction and picture reconstruiction processes. The background is about five times stronger than the pinhole signal at the center of the pin. Near the top and bottom of the pin, the background is an even greater fraction of the total response. Amplified statistical fluctuations [LU-80] and background anomalies [N0-80] could account for phantom data. Also artifacts are present in the reconstructions used for determining radial expansion.

Additional causes for artifacts are the vidicon flyback trace, the gadolinium tags, and the requisite vertical blending of four overlapping pinhole images, each with separate positional-intensity variation. Another cause for artifacts or systematic discrepancies is nonlinear instrument response. The hodoscope data is corrected to a large extent for its supralinear Hornyak-button response on the basis of extensive experimental data, and the tandem proportional counters respond linearly over a wide range. Because of its lower signal/background ratio, the pinhole data is five or ten times more sensitive than the hodoscope to anomalous background effects. 
Operation and analysis difficulties. As a result of the PINEX-3 data exchange, it is becoming evident that there are special difficulties in collection and treatment of pinhole data. For example, considerable time must be devoted to changes, preparations, and calibrations for experiments. Such heavy dependence on staff-level calibration and preparation is not conducive to routine diagnostic operation. In addition, from the qualitative nature of much of the data presented, the processing and analys is of pinhole data is not without significant effort and uncertainty.

Experiment conditions. The PINEX-series experiments were characterized by three factors that tend to be particularly favorable to the pinhole camera:

(1). A high calibration factor ( $\sim 4 \mathrm{~W} / \mathrm{g}-\mathrm{MW}), 50$ to $100 \%$ greater than many TREAT experiments.

(2). A fuil slot through the core, compared to a half-slot under test conditions that require maximum energy input to the test sample.

(3). Application of the hodoscope under spatial resolution requirements that are at the limits of its installed design. The collimator used $(1.2 \mathrm{~m})$ is primarily intended for integral experiments with large fuel motion; a smaller collimator is available with better inherent resolution, but it could not be used in these experiments.

Although the first two factors improve performance of both diagnostic systems, the pinhole camera benefits the most from these favorable conditions because it has a poorer signal/background ratio and lower sensitivity. These favorable factors are not present in many experiments: multipin experiments generally have reactor-coupling factors two or three times lower; some experiments have nuclear-heated walls that greatly reduce the signal/background ratio; and for some shots there is a greater need for the energy deposition that would result from fuel that can be loaded in half of the slot.

Future refinement of data and analys is might improve results from both diagnostic systems. On the basis of documented functional standards, hodoscope sensitivity is usually sufficient as it stands; thus, hodoscope data treatment does not now, in general, take advantage of many applicable image-enhancement practices or pre-programmed correlation-analysis methods, similar to those used for processing pinhole-system data.

\section{Conclusions from hodoscope/pinhole comparisons}

After seven years of development dedicated to reactor transient applications (following many years of successful operation in weapons testing), the pinhole imaging system had approached a plateau in capability. It appears to have henefited from application of nearly all the detector capabilities, video-cameras, and instrumentation that are presently feasible. The extraction of improved results through computer processing is limited by the quality of the input data. At least an order of magnitude pinhole improvement is still needed in order to match hodoscope fuel-motion performance. A significant pinhole limitation is associated with its 
statistically inadequate performance at low power levels and at low-power stages of high-power transients.

PINEX-3 was the third comparative diagnostic experiment, but the first performed under uniform reporting conditions. To the extent that identified PINEX-3 events are contemporaneous and commensurable, the thermohydraulic and posttest examination data confirm hodoscope fuel motion results; otherwise, in places where confirmation does not exist or cannot occur, the hodoscope data was not invalidated. On the other hand, several effects reported by the pinhole are anomalous.

Details of pre-failure fuel pin motion were observed by the hodoscope, but none were reported for the pinhole. The pre-failure fuel motion within the $0.8 \mathrm{~mm}$ internal annulus to the test pin was detected by the hodoscope with mass resolution in the order of $0.05 \mathrm{~g}$, and axial spatial definitions of 0.5 $\mathrm{cm}$ were resolved in $12 \mathrm{~ms}$ intervals with $0.2 \mathrm{~g}$ sensitivity.

The hodoscope has detected validated internal fuel motion beginning with GE and HEDL experiments done ten years ago. In the 1971 HEDL experiment PNL 2-11, for example, the hodoscope data of the final stage of internal fuel motion within a 50-mil cavity were confirmed by post-transient radiography $[D E V-76 a]$. In the $1970 \mathrm{GE}$ experiment C4X, another example, preirradiated voids of 41-mil ID underwent time-dependent internal changes resulting in detected net mass transport through the central annulus [DEV-76a]. These data were confirmed by their consistency with outcomes observable by oiner means. For example, the discrete events eventually pass through phases, such as clad breach, that are subject to confirmation by thermocouple measurements, or the events terminate in a status that is subject to radiographic or physical examination.

Invalid data, data of questionable interpretation, and data with large uncertainties have been reported for the pinhole imaging system. Despite improvements in data processing, spurious fuel ejection has been interpreted from pinhole apparatus data, and pre-failure (internal) fuel motion has not been detectable.

The differences in outcome are not hair-splitting details; they can seriously affect the interpretation of expensive experiments. For example, in the PINEX test series the objective was to evaluate the role of internal fuel motion as an inherent reactivity-shutdown mechanism. The pinhole apparatus detected no internal motion in PINEX-2, but the hodoscope found extensive movement within the central annulus (Fig. III-14). Although the experimenter [SM-82] has capitalized on hodoscope and post-test observations that at least $10 \mathrm{~g}$ of fuel was ejected, hodoscope time-resolved data show that very little of this ejection occurred during the crucial initial phases of the transient. In PINEX-3, the pinhole originally implied even greater ejection, but the hodoscope showed none, and only $14 \mathrm{mg}$ was found in the examination. The clogging of the internal channel, which prevented ejection, made it necessary for the experimenter to take intu account the rate of TREAT reactivity increment in order to fit the model extrapolations. A far too optimistic projection of the initiating reactivity worth of the annular fuel would be inferred from the PINEX experiments if the pinhole results or the post-test examination were the only data available. 


\section{Indirect comparison with a coded-aperture system}

Neither the Fresnel-zone-plate nor uniformly-redundant-array coded apertures have been tested at the same facility as a hodoscope. Therefore only an indirect comparison can be made. Spatial resolution, data-collection intervals, and dynamic range are essentially independent of facility, whereas signal/background ratio, sensitivity, and mass resolution are facilitydependent. Available estimates that are based on performance in ACRR are included in Table $V-1$.

For e.cperiment and diagnostic reasons, fuel of high enrichment has been used at ACRR, a large energy deposition has been achieved by use of a flux trap, the impact of the capsule has been minimized, and the coded-aperture instrument system (CAIS) has been placed as close as possible to the fuel. The ACRR CAIS detectors have a relative solid angle of 12 compared to the position of the TREAT hodoscope. If installed at TREAT, considerable degradation in the coded-aperture system performance would be likely.

Proof tests of the Sandia system under combined transient conditions have indicated a low signal-to-background ratio, which is inherent in the codedaperture imaging concept with a reactor environment and in the use of fission gamma rays for imaging. Although the high imaging efficiency of the aperture permits a strong signal at the detector, the systematics of gamma-ray interactions results in a considerable background. Considerably improved over the previous value of 0.05 with the Fresnel-zone plate, the observed S/B is reported under transient conditions to be 0.25 for the new coded aperture. These would probably correspond to a S/B of 0.1 at TREAT.

Fuel energy deposition in a $4000 \mathrm{~J} / \mathrm{g}$ 6-second transient in ACRR somewhat similar to PINEX-3 was roughly $200 \mathrm{~J} / \mathrm{g}$ during a $200 \mathrm{~ms}$ interval for which a fue]-pin reconstruction was reported [ST-80]. The image as presented is irregular and al ready near the limits of sensitivity. Allowing a factor of ten to subjectively estimate sensitivity at a comparable level of pin threshold as applied in PINEX-3, the threshold would then be $20 \mathrm{~J} / \mathrm{g}$ in ACRR, an order of magnitude less sensitive (at best) than the pinhole-imaging system at TREAT. If one took into account relative solid angles, both diagnostic systems operated at TREAT would be another order of magnitude more sensitive than the coded aperture if they were situated at ACRR.

Adequate information on the dynamic range for the CAIS has not been published and it is difficult to estimate. Because of similarities in electro-optical conversion, the coded aperture should not have a wider dynamic range than the pinhole.

Because of this commonality with the pinhole in dynamic instrumentation, the coded aperture is evidently subject to difficulties arising from artifacts, discontinuities, and non-linearity.

In order to achieve adequate signal intensity, coded-aperture resolution has been sacrificed. Redesign of the system has resulted in a delay in achieving fuel-motion data. Now under development is a new design [ST-82], intended to reduce instrumental background though still without improving the scene-related background. Considerable improvement in CAIS sensitivity has recentiy been reported [KE-82]. 
Coded-aperture systems, because of difficulty in obtaining imaging detectors that adequately exclude gamma rays, inherently suffer by signal/background ratios worse by at least a factor of two compared to fastneutron detection.

\section{Flash radiography}

Although exploratory results under stationary fuel conditions have been impressive, transmission methods have certain inherent and technical problems that would need to be resolved. Flash $x$-ray systems operating with a repetition rate of at least $1000 \mathrm{pulses} / \mathrm{s}$ have not been demonstrated with the beam current and reliability required for use in transient reactors. None of the testing that has been carried out for flash-radiography has been conducted in prototypic environmenis where the background radiation varied with the reactor power. Moreover, reliable and interference-free operation of the accelerator would be required in conjunction with the nonreproducible reactor fuel tests. High-energy x-rays cannot be used to directly distinguish between fuel and steel. Beam divergence adequate to cover a large vertical field of view (and aspect ratio) is a special problem for transmission systems.

The requirement of a full slot through the core in transmission systems tends to exact a high penalty in core reactivity -- hence test-fuel energy deposition is reduced. For new reactors this may not be insurmountable, but for retrofits to existing reactors, the reactivity and financial cost may be too severe.

If the fuel being tested had a large enrichment gradient, or if there were a very large number of pins in a subassembly, flash $x$-ray systems might in principle be a better diagnostics tool than the hodoscope. Whether the practical limitations could be overcome remains to be seen.

\section{E. In-core detectors}

None of the in-core/in-test detector techniques have proven successful for a number of reasons. Detectors located in the test section will fail before much of the significant fuel motion takes place; also they compete for precious space and instrumentation leads with thermocouples which are useful for many purposes, including indication of cladding failure. If the detectors are located out of the test capsule, they are much less sensitive to localized fuel motion and radiation effects. In either case, their response is largely ambiguous because they cannot differentiate between neutron flux changes that occur as a result of local fuel, sodium, or steel changes. Normalization for power level is difficult because non-linearities can be in the scale of signal changes $[S A-82]$. 


\section{F. Comparative performance}

1. Specific diagnostic system quality indicators

Although the pinhole diagnostic system has been making noticeable improvements, the direct PINEX-3 data exchange shows that its performance still fails to match the capabilities of the hodoscope in qualitative as well as quantitative fuel-motion monitoring of transient data.

Unfavorable theoretical and developmental data indicate that the codedaperture system, after seven years of development, has not met initial expectations.

For the $A X-1$ three-pin experiment, a non-redundant pinhole array [BE-76a] failed to detect any of the fuel motion, while the hodoscope obtained considerable detailed data. An asymmetric orientation of the fuel within the test capsule placed the pinhole array at a disadvantage, but had the diagnostics systems been interchanged in position, the hodoscope would still have been able to achieve usable data.

The results of these comparisons are summarized in Table $V-1$. Because comparative data is derived from highly favorable experiment conditions, it is likely that the alternative imaging systems will not supply any useful data under more demanding flux, slot, and test conditions.

\section{TABLE $V-1$}

Demonstrated Diagnostic System-quality Indications for Transient Data

Signal/background ratio

Pin visibility threshold, $\mathrm{J} / \mathrm{g}$

Spatial resolution (dynamic)

vertical, $\mathrm{mm}$

horizontal, $\mathrm{mm}$

central pin zone, $\mathrm{mm}$

reflector/plenum, mm

Data collection intervals, ms

Mass resolutign, $g$

Dynamic range

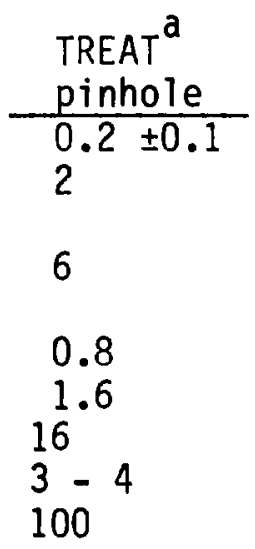

TREAT $^{\mathrm{a}}$
$\frac{\text { hodoscope }^{2}}{1.9 \pm 0.1}$
0.02
6
$<0.8$
$<0.8$
2
0.2
10,000

\section{Notes}

a: from direct comparison in PINEX-3 experiment

b: from indirect comparison

c: for correlated fuel motion

d: not fully exercised in PINEX-3

e: $1 \mathrm{~ms}$ possible but not practical because of statistical limitations

$f$ : estimated from $5 \mathrm{~J} / \mathrm{g}$ demonstration

g: at $1 \mathrm{~J} / \mathrm{g}$ threshold 


\section{Analog data}

Both coded-aperture and pinhole devices accumulate results in the form of analog images stored on framing-camera film and/or video-tape recorders. The analog-to-digital conversion step runs the risk of losing significant information or introducing artifacts. There is a comparable problem in reconverting from digital to analog data; however, of greater value to the customer are the quantitative results directly derived from the digital data. The loss in visual appearance that could result from conversion of digital data to analog images can be tolerated because analog reconstructions usually serve no quantitative purpose -- they are qualitative illustrations for the purpose of communicating impressions rather than numerical values.

One of the inherent prohlems with an analog data acquisition method is that digital conversion of recorded analog data must take place to achieve quantitative results. In principle, systems with limited dynamic range cannot yield high-quality results in the analog-to-digital conversion. Front-end digitization of data, which is carried out for the hodoscope, is directly amenable to providing the quantitative information needed for application in reactor safety analysis. In any case, state-of-the art analog pictures can be derived from any diagnostic system, as depicted schematically below:

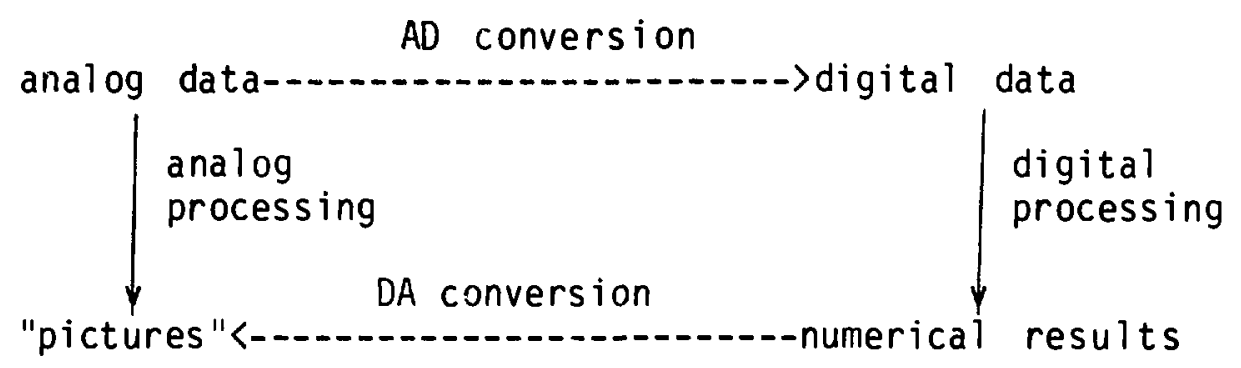

"Pictures" constructed from analog data systems are usually quantitatively inadequate and sometimes qualitatively misleading. Conversion into useful quantitative data requires an unavoidable digitization step that introduces a new source of error. Moreover, with poor signal-to-background ratio, unprocessed pictures are not dependable indicators of fuel motion.

Confusion sometimes arises about the relative value of qualitative and quantitative attributes of a diagnostic system. Preseniing a visual "picture" in pleasing form is useful for qualitative understinding of the progression of events. These "pictures" communicate the overall ieatures of an experiment, particularly to persons who are not directly invelved in quantitative analysis. Attributes that permit quantitative estimation of the key observables are necessary to the experimenter or person inputting data into a fuel motion modei. While both types of attributes are useful, the stress has always been on quantitative data --with a secondary role for subsequent pictorial reconstruction.

In the mid-1960's, developmental hodoscope data was taken in analog fashion on film; starting in 1969 it was recorded by placing digital data on film. Now hodoscope data is stored directly on digital magnetic media. The process of digitizing analog data has been less effective than the process of recording digital data. It is possible to derive, when warranted, visual 
reconstructions from digital data without meaningful loss of image information quality. It is better to have high-contrast digital data for generation of visual reconstructions than to start with low-contrast images that need enhancement [TW-82]. With good-quality data, sensitive digitalsubtraction techniques [BR-82] are applicable.

\section{On-line data observation}

On-line data observation is generally impractical for most reactor-safety experiments due to their brevity $(0.1 \mathrm{~s})$ or the rapidity $(100 \mathrm{~m} / \mathrm{s})$ of a single event during the experiment $(1-10 \mathrm{~s})$. Rapid-turnaround of data after an experiment is useful -- provided it can be done unambiguously. Although no particular diagnostic system would hold an inherent advantage over the others in turnaround time, practical difficulties in data reconstruction weigh heavily.

\section{G. Optimi $\angle a t i o n$ of performance}

In terms of specific quality indicators, comparative performance at the time of the PINEX-3 data exchange may be summarized as follows: hodoscope signal-to-background and mass resolution appear to be an order of magnitude better than the pinhole. Limiting mass resolution of the coded aperture at ACRR might eventually approach that of the hodoscope, but this has not been demonstrated in practice. Hodoscop time and spatial resolution are as good or better than alternative imaging devices. In sensitivity and dynamic range, the hodoscope has two orders of magnitude advantage. In addition, hodoscope data appears easier to process, analyze, and utilize and is evidently less subject to spurious effects.

The hodoscope tolerates very high count rates in each channel because it has a segmented design. Each detector can be selected and operated so as to maximize efficiency and rinimize background. As a result of this simultaneous optimization, the hodoscope has a very wide dynamic range.

The better resolving power of the hodoscope comes because it is tailored to background and test surroundings. Hodoscope resolution principles allow surprisingly high fuel-motion resolution and rather simple development and verification. Moreover, a one-channel static test is sufficient for hodoscope proof-of-principle.

The segmented nature of the hodoscope not only allows a building-block approach, but it is directly compatible with digital techniques of data transmission, recording, and processing. Although this would not necessarily be the foremost factor in instrumentation design, it has proven to be an extremely efficient approach for the hodoscope, and its absence in other diagnostic techniques can be a major limitation.

Another powerful feature of the segmented, multi-channel approach is the wider range of options available in choice of detector and signal-processing electronics and in the setting of operating parameters such as energy thresholds. Alternative coded-aperture and pinhole systems that cannot be segmented must be tested at full scale in order to properly evaluate all 
factors. Because development, design, and implementation of multi-channel systems are straightforward, costs of installation, operation, and analysis have proven to be lower than alternative approaches.

All of the non-hodoscope imaging systems depend on some type of electrooptical detection system. These have serious limitations when applied under conditions for which they were not designed. The number of pixels must be sufficient to provide adequate resolution for small image areas of large aspect ratio, the linearity of the device should be within a few percent (or well characterized) for a dynamic range of 1,000 to 10,000, and the quantum efficiency per pixel ought to be sufficient to avoid a statistical choke at the beginning of the conversion.

A thorough understanding of background and systematic effects upon signal is important in achieving quantitative data -- the major goal of the reactorsafety experiments. These are difficult effects to evaluate, and more attention is being turned to them. The magnitude of some corrections does differ between transmissive and emissive systems, but none of the alternative systems are immune from systematic or statistical factors [DEV-82a].

As larger volumes of data and more data dimensions are experienced, more sophisticated data-reconstruction processes, including three-dimensional tomography, are invoked. Inevitably judgment must play a role in treatment of any set of data. But one diagnostic method can be more susceptible tc artifacts and judgment than another. Artifact susceptibility might be the major limitation of analog systems, such as the pinhole and coded-aperture, because of the large background fraction they must subtract.

No system is immune from technical corrections to the data. There are inherent errors common to all diagnostic systems that use the same source. For example, hodoscope, pinhole, and coded-aperture systems rely upon emission of radiation from the fuel. Flash $x$-ray systems would not be subject to the same inherent corrections because they are dependent primarily on transmitted radiation; however, they are affected by other correction factors and errors. 


\section{OTHER APPLICATIONS}

Although only three nuclear reactors have in-pile cineradiographic instrumelitation, evaluations have been carried out for the purpose of obtaining either high-resolution or coarse-resolution diagnostics in other reactor facilities [DEV-82d]. Furthermore, the data collected from detectors used in cineradiography provides not only time-resolved spatial distributions, but it can be used in other ways important to the experiment program.

\section{A. Other reactors}

The Engineering Test Reactor (ETR) has been used as an irradiation facility for loss-of-coolant reactor-safety simulations with test bundles containing up to 37 pins. Although the experiments were eventually conducted without highresolution diagnostic devices, two alternative designs of hodoscopes were considered. Figure VI-1 shows an isometric view and the layout within the reactor vessel and shield. With two hodoscope collimators at right angles, threedimensional information would have been provided. A design for a coarse-resolution hodoscope, which would yield only axial fuel-motion data, was carried through conceptual design stages. In the actual sodium-loop experiments at ETR, incore fuel-motion detectors were used, but without success.

A coarse-resolution hodoscupe was also considered for a safetyresearch modification of Experimental Breeder Reactor (EBR-II), especially since only minimal changes to the pressure vessel were allowed. As the layout in Fig. VI-2 indicates, a narrow slot through the core would permit fast-neutron detectors to be in a shielded position at the outer periphery of the core. This compromise would allow relatively coarse onedimensional axial resolution of fuel movement in the central zone, within :ne design constraints of the - ese:or modification.

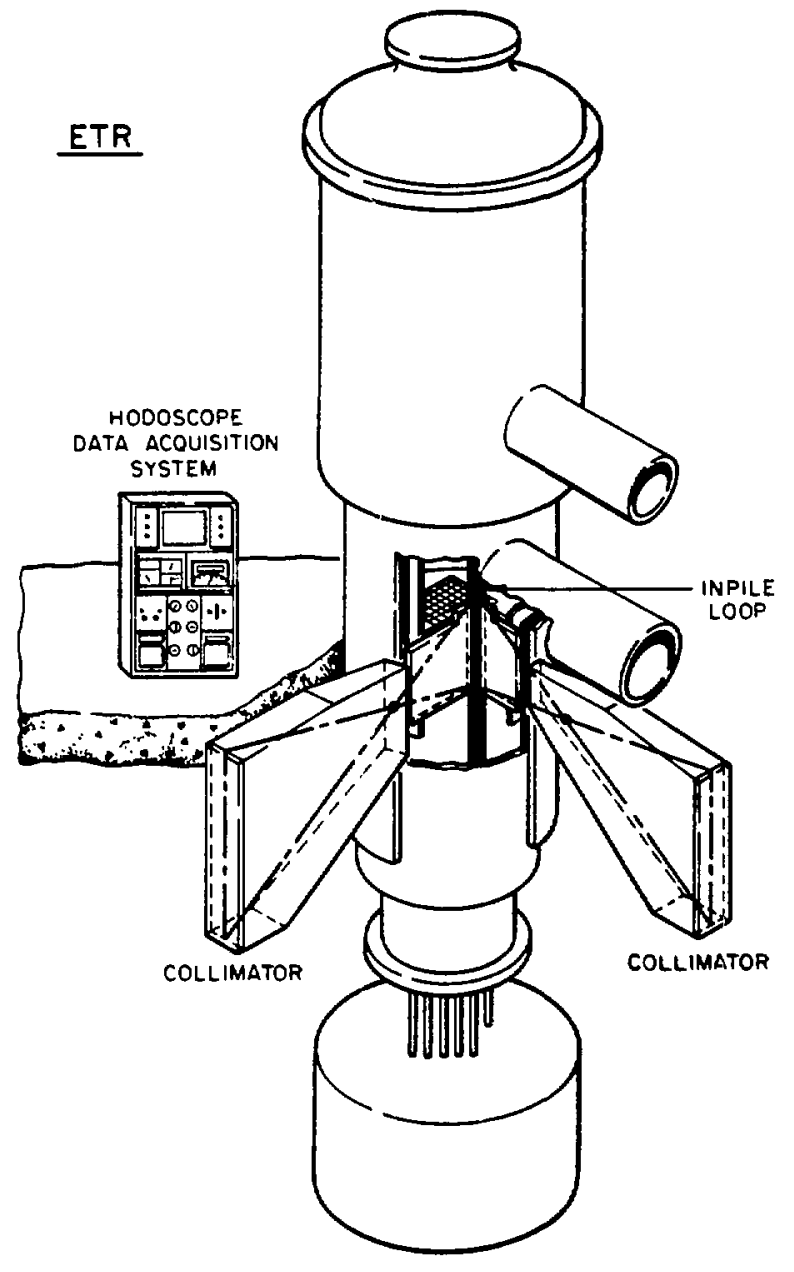

Fig. VI-1. High-resolution hodoscope proposed for ETR (isometric view). 
Concepts or designs have been carried out to various levels of detail for other existing or planned reactors [DEV-82d].

\section{B. Time-integrated radiography}

Cineradiography is based on radiographic exposures obtained or projected at repetition rates sufficient to avoid motion blur [DEV-82b]. A single-exposure, time-integrated radiograph is a building block for cineradiography. In the absence of moving objects, time resolution can be traded in favor of higher image quality due to better data statistics and recording media.

One technically feasible possibility for application of accelerators in the reactor-safety program is in transmission radiography of stationary objects, such as full-subassembly tes sections in thick enclosures. Accelerators, though, might not compete well on the basis of cost effectiveness with existing reactors and other sources of penetrating radiation $[\mathrm{RH}-82 \mathrm{c}]$.

Another approach to timeintegrated radiography, one that utilizes existing facilities, is hodoscope in-situ fast-neutron scanning (Fig. VI-3). In this operating mode [DEV-76b, DEV-78, DEV-82c, RH-77b] the hodoscope is scanned =:th horizontally and vertically to increase its effective spatial resolution for stationary objects. The operation is carried out at low steady-state reactor power, and no fuel motion is likeily to occur. This scanning operation takes advantage of the equivalence of relative motion between the collimator and the fuel object. Thus the results may be inturpreted directly to define fuel-motion spatial resolution, as well as provide pre- and post-test radiographic data.

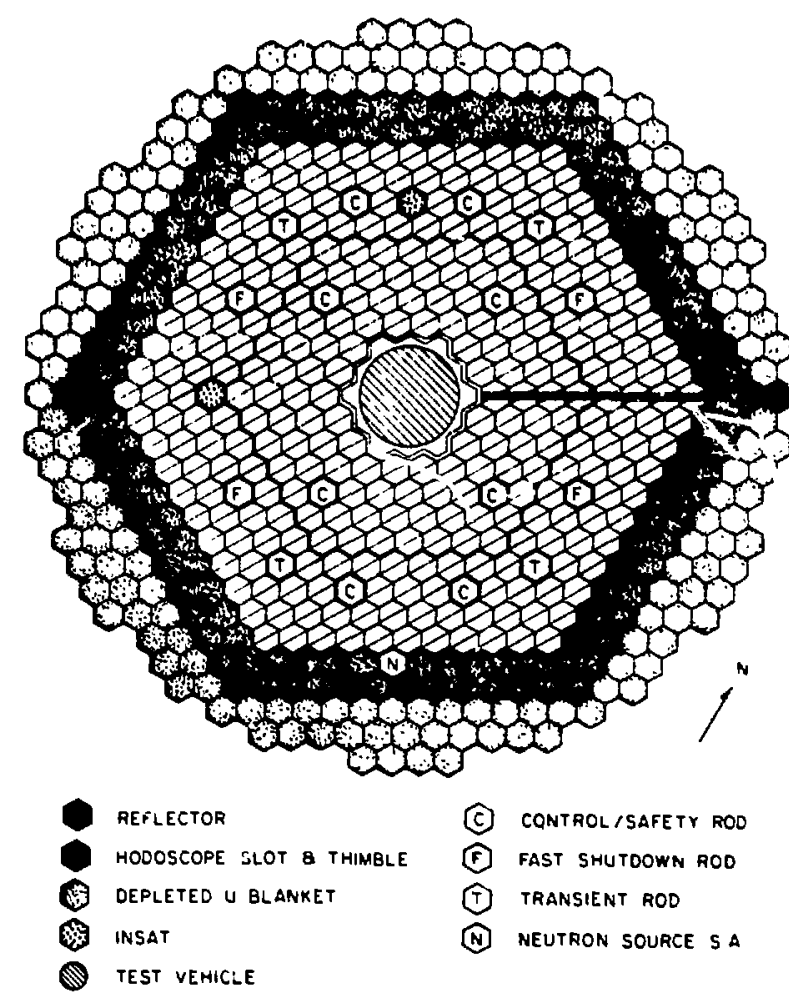

$\underline{E B R-I I}$

Fig. VI-2. Core layout for EBR-II modified to accept a coarseresolution hodoscope. Only axial fuel motion could be observed through the slot which would be terminated near the reactor vessel wall by a partially collimated single column of fission counters. The detectors and slotted fuei elements could all be loaded in the core without reactor modifications. 


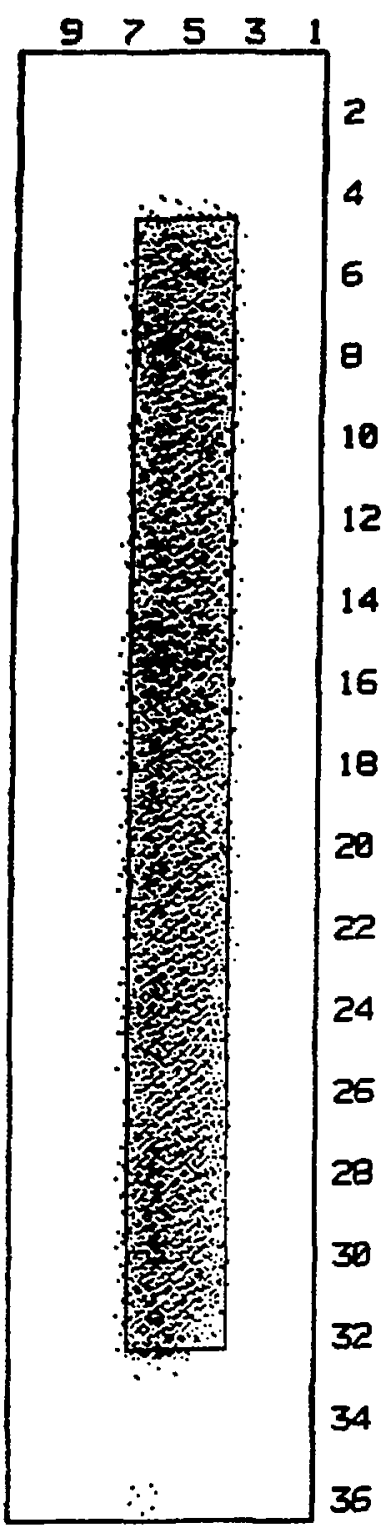

a

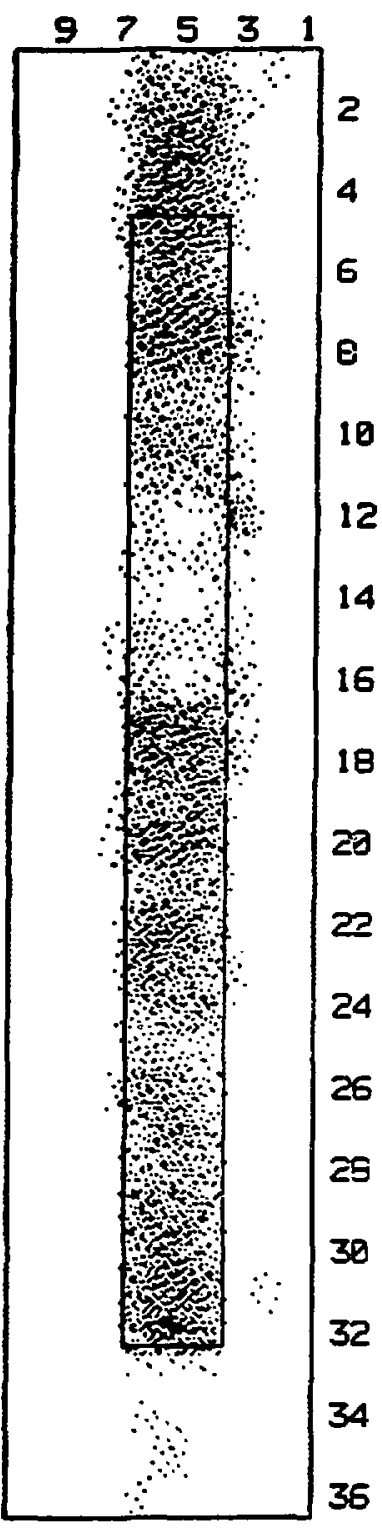

b

Fig. VI-3. Digital reconstruction of fast-neutron hodoscope scanning radiograph. Comparison of pretest (a) and posttest (b) fuel distribution, indicating substantial relocation. The darkest areas correspond to higher fuel concentrations. A 7-pin bundle was originally within the dark rectangular zone of the pretest radiograph. 
Being sensitive to fission rate, fast-neutron detecton offers a means of differentiating between fissile and fertile fuels and a means of confirming pellet enrichment. In addition to fuel distribution, it is possible to simultaneously sort out steel relocation using the gamma-ray hodoscope system, provided sufficient steel displacement takes place.

The application of deconvolution techniques to hodoscope scan data is illustrated in Fig. VI-4. Here, for the single pin PINEX-3 experiment, the $0.8 \mathrm{~mm}$ central void is identifiable after deconvolution. These methods illustrate the capability of correcting for instrument characteristics to obtain an improved transformation to the original object configuration.

Hodoscope detectors respond to fuel movement across the viewing region of any detection channel. Because of this, the hodoscope is optimized for dynamic effects, in contrast to other imaging systems which are optimized to record static images. Hodoscope capability to resolve motion in increments that are much smaller than the interdetector pixel separation has often not been appreciated.

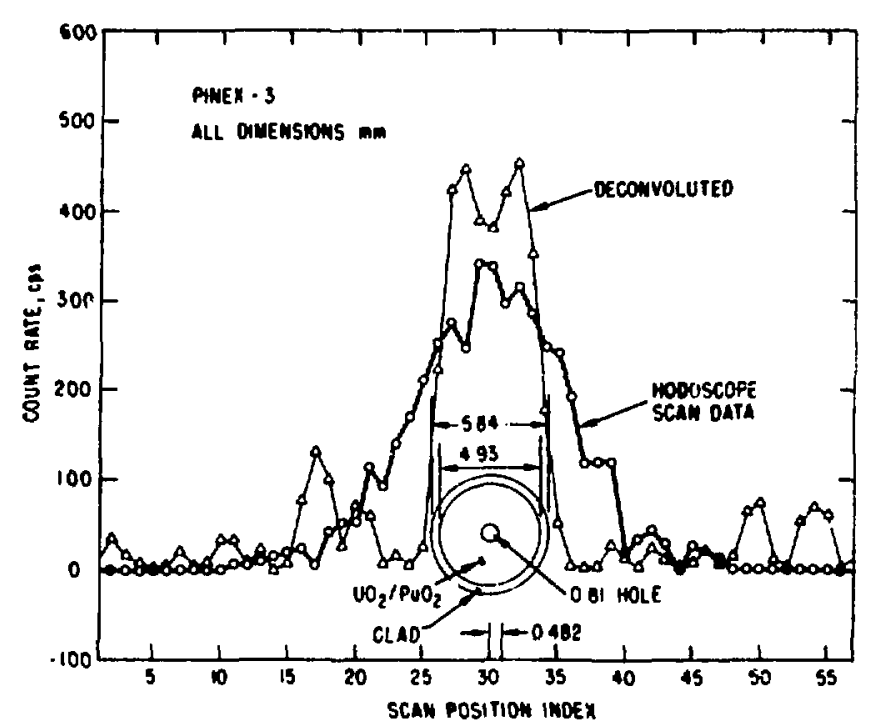

Fig. VI-4. Unconvoluted and deconvoluted scan-data comparison. The background-reduced pretest hodoscope scan of the intact fuel pin does not immediately suggest the $0.8 \mathrm{~mm}$ hole in the fuel pin, but the deconvoluted image that takes into account the collimator response function shows an appropriate dip at the center.

Because relative motion is the critical effect that induces signal changes that are measured by the hodoscope, it does not matter whether the fuel moves across the viewing pixel of a collimator channel or whether the pixel is scanned past stationary fuel. With this equivalence, it is possible under low-power steady-state reactor conditions to measure and verify the theoretical capabilities of the hodoscope; it is not necessary to drive the fuel to destruction for this purpose. With a fuel sample loaded in the test region of the core and with the TREAT reactor brought up to $80 \mathrm{~kW}$, a power level not likely to induce damage to the fuel, the hodoscope collimator is scanned across the object plane to produce a full profile of the source in about a half hour. In addition to aiding diagnostic development, this procedure has been routinely practiced for alignment of the collimator before a transient and for test-fuel characterization after an experiment. The result may be reconstructed into an image that corresponds to a fast-neutron radiograph, as shown in Fig. VI-4, which compares the post-test distribution of fissile material with the pretest intact distribution. 
Originally only horizontal hodoscope scanning was mechanically feasible. In this mode, interchannel efficiencies could be calibrated using a fueled or unfueled test vehicle. The axial flux gradient cancels out, so that a reconstructed image wou?d bo free of the effect. Adding a vertical scanning capability has provided a way of linking all detectors with each other, enabling the determination of efficiencies that may be applied to transient data as well as normalization of scan data. This capability has recently been implemented [FI-82] but not fully exploited.

For experiments in which the location of initial fuel failure or other information dealing with the axial location of fuel movement is important, the vertical flux profile along the test vehicle is a measurement of interest. This flux profile is a function of reactor induced effects, including control-rod position, and therefore requires direct measurement under correct conditions. Scanning operations may be carried out to extract this data under fixed, steady-state conditions.

Before and after experiments, a corresponding radiographic operation has been tested by the pinhole apparatus, in which a film plate is use as the recording medium. No movement of the pinhole collimator is necessary during exposure. The time-integrated exposures appear to have relatively high contrast and good resolution.

The resulting images differ in the following ways: (1) The pinhole imaging is sensitive to gamma rays from structural elements, as well as neutrons from fue 1; whereas the hodoscope detects primarily fast neutrons from fuel. (2) The pinhole film data appears similar to other traditional radiographic images, although limited in resolution by the characteristics of the imaging device; the hodoscope data must undergo a process of image reconstruction. (3) The hodoscope conducts its scanning operation by discrete digital methods, which provide initial digital data that can be directly used for quantitative purposes; the pinhole analog image must be digitized subsequently for quantitative results. (4) The pinhole radiograph appears to have better resolution than the hodoscope reconstruction.

Spatial resolution of the resulting image is limited by the characteristics of each system, except that pinhole's film data has better resolution and contrast and is freer from artifacts than its transient data because of two factors: (1) the superior recording qualities of $f$ ilm compared to the high-intensification TV camera, and (2) the recording of delayed gamma rays which are not available during brief transients. These two factors combined probably improve the signal-to-background ratio of the pinhole film image by a factor of at least two compared to its transient data.

\section{Reactor-power monitoring}

Although cineradiography appears to be confined to reconstruction of timeresolved spatial images of an object or its density variations, the test object may also be considered a means of transferring other information of interest. In nuclear reactors, any object viewed at the center of the reactor would have interactions with and reflect the incident neutron intensity and spectrum. If the object is fissionable, incident thermal neutrons are exchanged for fission neutrons and gamma rays. The measurement 
of these particular test-sample and reactor-flux properties is of growing importance in reactor-safety experiments.

of primary interest is the reactor flux present in the entire field of view during an experiment. The magnitude of this flux can vary in time and space. With an unfueled sample placed at the center of the core, direct-view cineradiographic instrumentation can observe the neutron or gamma flux during the course of a transient. The hodoscope, for example, normally observes the fast-neutron flux above $0.5 \mathrm{MeV}$, which includes fast neutrons scattered from the sample. If fuel is inserted at the central position, then the coupling of reactor neutrons to the fuel is also observed. It is the capability for direct monitoring of the pin power and of nearby core power in separate detection channels that is important.

Control rods produce time-varying asymmetric flux patterns and core temperature changes also induce important axial and radial flux shifts [DEV82c]. The fuel may undergo a different temperature history, and because of its composition, its power coupling is likely to change during an experiment. Because of spatial variations across the core, the reactor instrumentation that is normally located in the reflector does not accurately detect the flux distribution at the center of the core nor the power coupling of the fuel. Consequently, only the direct-view imaging instrumentation can measure the correct neutron properties of the test fuel.

In order to sort out variations in flux or power coupling from fuel motion, analysis and calibrations must be pursued. Experiments conducted with dummy capsules under prototypical operating conditions divulge the underlying changes in source term. The hodoscope has detected axial flux tilts reaching extreme values of $10 \%$ during a TREAT transient, which is corroborated by integrating monitor wires. The wires have also substantiated that the hodoscope tracks the flux accurately while the reactor instrumentation must be corrected by as much as 30\% in TREAT and $60 \%$ in CABRI. Valid measurements of the the transient-correction and power-coupling factors are important in planning and interpreting reactor-safety in-pile experiments.

\section{Ex-vessel monitoring}

A possible use for hodoscope-type collimated systems might be in ex-vessel monitoring of core properties. Operating power or test reactors that cannot accept in-core diagnostic systems, largely because of radiation damage, could be equipped with collimated fast-neutron detectors embedded in the reactor biological shield. Because such systems would have to detect uncollided through the thick reactor vessel and at least partially through a large core, resolution would be limited by an integral along the line of sight of each channel. Nevertheless, the possibilities exist in a role not met by other detectors for an indestructible monitor of subcritical power, core power, and coarse fuel location monitoring -- preceding, during, and after a possible core meltdown [DEV-82d].

Application of such a monitoring scheme was explored for TMI-2 in the post-accident recovery phase in order to characterize the fuel reconfiguration. Although some methods using in-core detector tubes are likely to have 
been more successful, ex-vessel collimated fast-neutron techniques, subject to more extensive study, might have been able to provide useful core condition data. 


\section{IMPROVEMENTS}

Cineradiographic instrumentation applied to nuclear-reactor safety is undergoing progressive improvement to meet challenging experimental situations, much of it occasioned by tests being performed under more prototypical conditions with larger fuel assemblies. Aside from such improvements to the diagnostic hardware, much more substantial benefit can usually be derived by reactor modifications that increase power coupling or diminish background.

\section{A. Two-dimensional diagnostics}

In order to improve capability for two-dimensional monitoring of flux and fuel distributions, some straightforward instrumentation improvements have been defined. These are directea towards increased mass accuracy and coping with larger test fuel assemblies.

1. Hodoscope improvements planned

In view of the satisfactory performance of the TREAT hodoscope proportional counters, the maximum array of proportional counters is to be implemented. This will permit better statistical and systematic evaluation of background surrounding the fuel. Experience indicates that about twice as many detector columns should be devoted to unambiguous background vs. signal monitoring, in order to minimize systematic and statistical effects of background subtraction.

Improvements that are projected further in the future include equipment and software for better analysis, and detectors with improved efficiency and signal/ background ratio for use in scheduled tests where thicker test vessels surround the fuel sample [EV-79a, EV-79b, FI-79].

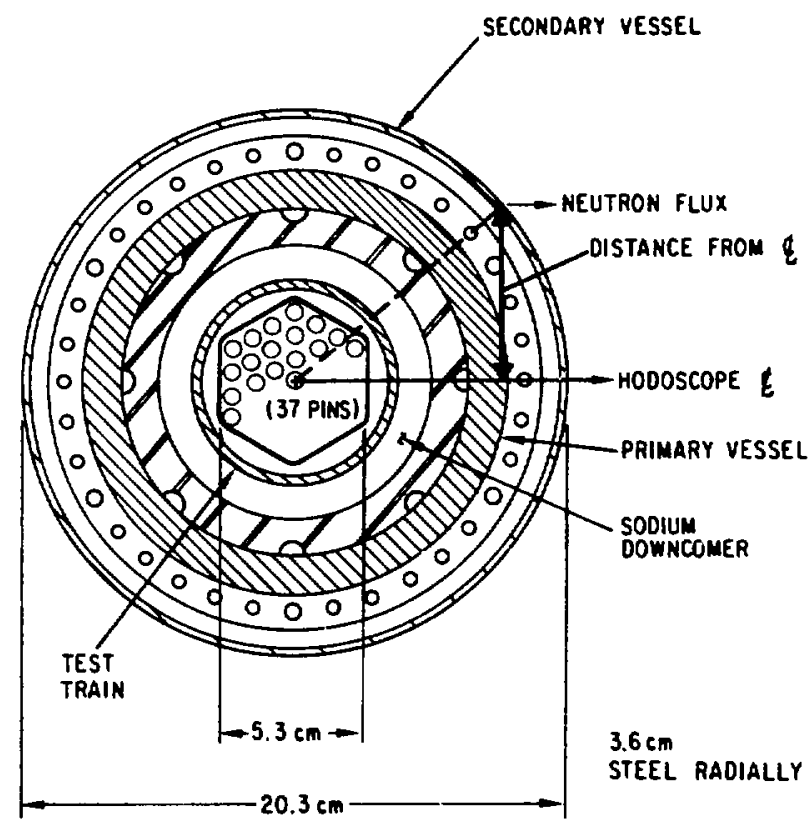

Fig. VII-1. Cross-section of planned 37-pin subassembly for TREATupgrade. A radial thickness of over $5 \mathrm{~cm}$ of sodium and steel surrounds the fuel pins.

With the advent of an upgraded TREAT, certain additional diagnostic needs have been recognized. One of these includes higher resolution in the central detector zone in order to have better detection of axial expansion and cladding breach. Another is a broader field of view in order to cover 37-pin fuel bundles (Fig. VII-1) and to allow coverage of a larger range of axial fuel expulsion. These conditions can be met on a cost-effective basis by moving the collimator away from the TREAT reactor about $20 \mathrm{~cm}$, adding three or four additional columns of collimation and detectors, and remachining the slots to cause uniform interchannel overlap. 
An upgrade of TREAT will also bring about tests in which fuel within a 37pin bundle will have varying enrichments. This will have a substantial effect upon quantification of fuel movement. A study is underway to evaluate the possible time-resolyed detection of enrichment-gradient changes as a result of changes in a $235 \mathrm{U} / 239 \mathrm{Pu}$ fission ratio. Self-shielding and self-absorption corrections will also become substantial, and efforts will be necessary to accommodate these effects through iterative computational corrections [AL-82]. The combined complexity of these effects is likely to necessitate greater reliance on image-restoration techniques.

Greater emphasis will be placed on the use of the hodoscope as direct-view instrumentation for various power-coupling-factor changes in TREAT. At CABRI and TREAT, investigation of fission-product monitoring is underway through neutron or gamma techniques.

There is a continual program of improvements for the sake of reliability. In fact, a major portion of the apparent complexity and expense of the hodoscope system is aimed at achieving dependable performance. A strong degree of operational assurance is warranted because of the high cost and long lead-times associated with destructive experiments. Consequently, quality contrcl is routinely exercised in hodoscope procedures and hardware, and considerable redundancy has been designed into the entire diagnostic system. For example, the two fast-neutron detection systems, the proportional-counters and the Hornyak-buttons, are completely isolated in terms of power supplies, electronics, and recording apparatus.

\section{Other diagnostic techniques}

Coded aperture developers at Sandia are investigating a cross-slit pinhole configuration and a uniformly redundant array in order to overcome instrument background problems.

Position-sensitive detectors are the common aspect and weakest element of the coded aperture and pinhole imaging systems. Higher efficiency and better discrimination against neutron would help; so would a wider linear dynamic range for the image transfer system, be it from vidicon to film or raster scan.

Because fuel bundles are over ten times larger than their width, the aspect ratio is awkward for the round or square image transfer systems, often resulting in their vertical stacking. Also the image transfer unit is critical to performance, allowing little latitude for redundancy. Major improvements in all components will be needed in order to meet large bundle test requirements.

For accelerator imaging to be acceptable, a demonstration under actual reactor conditions would be necessary. Before such a trial, an acceleiator with adequate current, repetition, rate, energy reliability, anu compatibility would have to be built. 
B. Three-dimensional tine-resolved data

Three-dimensional time-resolved data becomes more useful as fuel pin bundles become larger. Ninetydegree orientation of two hodoscopes would suffice. While this is functionally possible at TREAT, core-loading considerations make it difficult. A new safety test facility (STF) had been studied in which three hodoscopes were oriented at $120^{\circ}$, providing adequate coverage for three-dimensional reconstruction [MI-77, PE-78] (Fig. VII-2).

Although the alternative coded imaging techniques are theoretically capable of three-dimensional viewing from a single angle, in practice useful resolution in depth has been unachievable. With larger bundles, some coarse depth resolution may be useful at a single angle. The safer approach is to use several viewing angles.

C. Three-dimensional static tomography

At low-power reactor operation, when no fuel motion is taking place, the test bundle will be rotatable within the upgraded TREAT reactor core while the fuel is irradiated. As a result, with its collimator stationary, collecting hodoscope data at each angle of fuel-vessel rotation, three-dimensional fastneutron static tomographic radiography could be performed [DEV82f]. In-situ measurements of this sort have the advantaye of extensive characterization of the fissile component of the experiment before possible disturbance upon removal and transfer can take place.

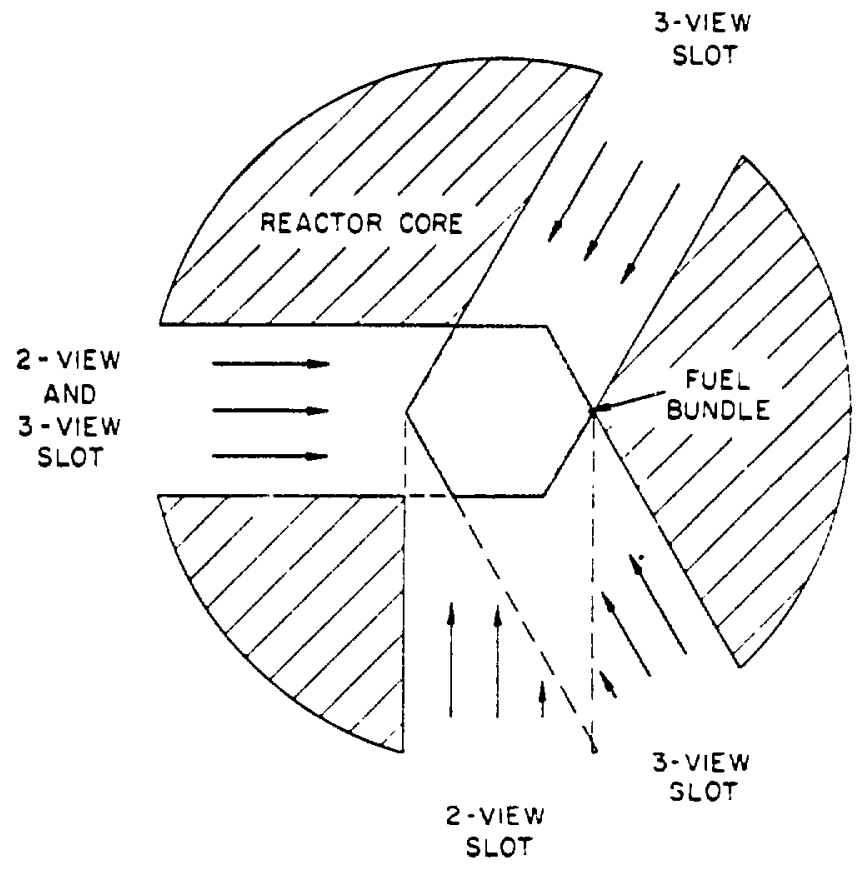

Fig. VII-2. Three-dimensional hodoscope cineradiograpy. Conceptual plan of 2- or 3-view slot arrangements for hodoscopes that would provide time-resolved tomographic data in a proposed fastreactor safety test facility (STF). The number and width of the slots affects the capability of the reactor to deliver a large and uniform radiation burst to the test fuel bundle.

A related development being planned is that of fuel-pin plenum radiography. If fuel is ejected during a transient from the original fuel zone, it will disperse into the upper and lower plena, which are outside the $1.2 \mathrm{~m}$ field of view of the hodoscope. The first logical opportunity for post-transient radiography of the plena is while the test section is being 
withdrawn from the core of the TREAT reactor. Detectors can be positioned so as to detect fission-product decay gamma rays emitted from the entire test section after the experiment. Tomographic images can be obtained by detector rotation. A hodoscope-type scanning system (Fig. VII-3) appears most promising, although pinhole-type apertures are also candidates for this task.

The gamma scanning capability has been developed for TREAT upgrade in which 37-pin bundles will be tested. After a transient experiment, as the test vessel is slowly withdrawn from the reactor, gamma rays from fissionproduct decay will be detected by an array that views each elevation at many angles. The result will be three-dimensional gamma-ray tomography of the entire vessel, including plena zones outside the hodoscope central field of view.

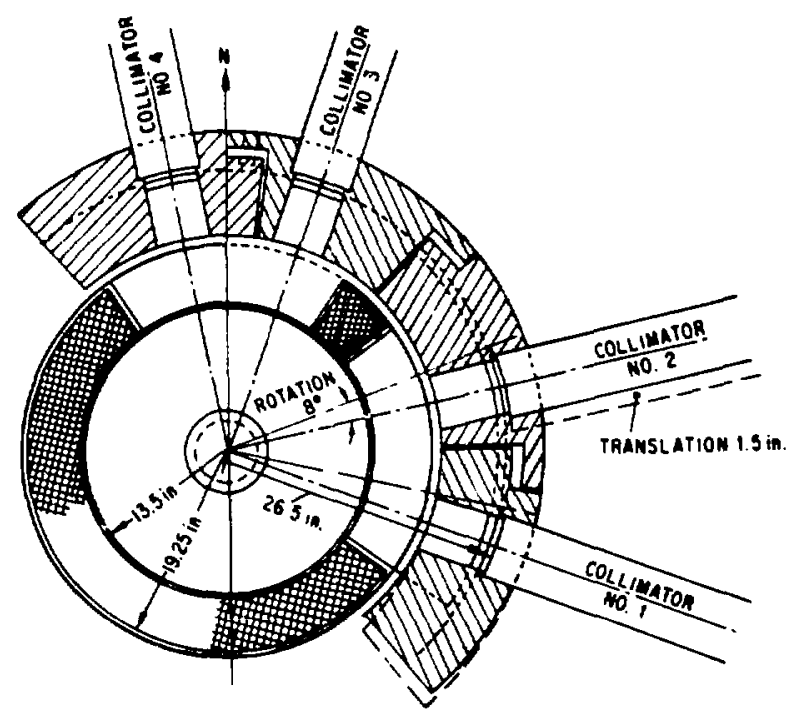

Fig. VII-3. Tomographic gamma-ray plenum scanning. Initial design of rotation and translation apparatus to permit tomographic gamma-ray scanning of a long test vehicle as it is slowly pulled out of the TREAT reactor. The four collimator assemblies, consisting of up to eight collimated detectors, can be moved so as to cover $180^{\circ}$. The test fuel would be contained within the center circle. 


\section{CONCLUSIONS}

Cineradiography is advancing with contemporary technological development, finding new applications in many fields. In cddition to military applications, particularly ballistics and explosive development, there are industrial and research uses for time-resolved radiography, especially in medical diagnostics.

A long-standing and important cineradiographic application has been in nuclear-reactor safety, where fuel-motion diagnostics has been applied to resolve time, space, density, and material parameters during destructive transient experiments. After seven years of intense parallel development, evaluation now reveals the asymptotic levels of performance for several types of diagnostic systems. In this regard, instrumentation goals have been met or exceeded by hodoscope-type systems. Very few of the critical diagnostic parameters have been matched by position-sensitive analog imaging systems, which have yet to detect details of internal-prefailure or multiple-pin fuel motion.

Because each detection channel can be optimized for selective response to fast neutrons, which give the most definitive and penetrating radiation signature of fuel, and because each channel can handle large count rates, the segmented design of the multichannel hodoscope has a high performance capability. This largely accounts for its good signal-to-background ratio and wide dynamic range. Despite being surrounded by two or more centimeters of steel, fuel mass resolution can be approximately 0.1 g. Spatial resolution, dominated by design limitations and by statistics, is better than $1 \mathrm{~mm}$ for horizontal and $1 \mathrm{~cm}$ for vertical fuel displacement. Data-collection intervals smaller than $1 \mathrm{~ms}$ may be used, although actual time resolution is likely to be larger because of the reciprocal statistical relationship to other resolution parameters. Adequate transient recording duration of digital data has been accomplished by the use of specialized magnetic disk and tape media. A high degree of redundancy has been built in to provide the needed readiness and reliability that has been experienced in association with expensive and nonreproducible fast-reactor-safety fuel-destruction experiments.

Whereas none of the alternative diagnostic systems are theoretically precluded from meeting the statistical conditions needed to differentiate between modes of fuel movement, only the hodoscope has been able to demonstrate in practice that such subtle phenomena as fuel breach and pin expansion can be reliably distinguished. In retrospect, the limiting performance factors for the hodoscope were established about a year after initial concept, whereas the position-sensitive devices have still not reached that level after more than six years of development.

Difficulties experienced in qualifying fuel-motion diagnostic systems alternative to the hodoscope may be understood in terms of the particular nature of the application. Imaging devices that are workable in other situations will not necessarily be successful at a reactor, particularly where the background becomes more significant than the instrument noise. When compared to collimated motion-monitoring systems, position-sensitive imaging systems appear to be fundamentally limited in transient reactor experiments. Some limitations are inherent and some are practical. The 
signal/background ratio for fast-neutron detection is a factor of at least two better than the gamma-ray detection to which the imaging systems are largely confined. Furthermore, high sensitivity to artifacts is an inherent feature of coded systems, particularly the Fresnel-zone plate [MC-82], because of the low signal/background ratio and the superposition of images. Artifacts that appear in image reconstruction can arise from nonlinearities in either signal or background or by the presence or introduction of systematic effects in the instrument. Relatively poor dynamic range is associated with position-sensitive image-transfer systems because of practical limitations. Unrecognizable coherent effects usually do not pass through hodoscope-type digital-data analysis.

Because of the context of practical experiment situations, spatial (and therefore mass) response is not readily and uniformly defined in fuel-motion diagrostics. Theoretical constructs of point- and line-response functions can liffer by orders of magnitude from realistic situations. Nowhere is this more apparent than in the significant discrepancies between demonstrated perfcrmance of the hodoscope segmented system as contrasted to the overly optinistic and unrealized resolution of pinhole and coded-aperture systems when projected to reactor-safety diagnostics.

Fuel-motion diagnostic systems can also produce static radiograpinic images. Coded apertures and pinholes can supplement their dynamic instrumentation with a sheet of $f i l m$ that provides a higher-quality timeintegrated image. Hodoscopes achieve a digital radiograph because fuel motion across a collimator projection is equivalent to a collimator projection scanned across stationary fuel. This procedure also lends itself to reliable hodoscope development scaled from single-channel tests.

Although time-integrated images from position-sensitive systems appear visually informative, particularly because some users are more familiar with analog presentation, their programmatic usefulness is yet to be established. Time-integrated radiographs are not a replacement for fuel-motion data when quantitative results under transient conditions are required. In any event, the hodoscope has underway improvements in data collection (more efficient detectors and better vertical resolution) that are applicable also to posttransient scanning, and the hodoscope has the yet to invoke capacity to improve data presentation by restoration algorithms. As a result, digital radiographic scan data can be improved in appearance while still yielding less ambiguous data than analog images.

Because fuel-motion diagnostic systems monitor emitted radiation proportional to neutron interactions in fuel or nearby materials, they can also act as direct-view instrumentation to measure fuel power coupling, transient-correction factors, and other reactor-flux-associated effects that are not well determined by other means. Inasmuch as only ex-core diagnostic instrumentation directly observes the entire fuel pin and assembly, it is likely to play an increased role in power monitoring and fabrication assurance.

Material-motion detection of a more general nature can be accomplished by hodoscopes with parallel-operation tandem detectors that respond specifically to certain materials. This method has been successful for simultaneous detection of steel movement. 
Hodoscopes have established themselves in termis of straightforward and predictable engineering design, fabrication, and cost. Not only are they functional at two reactors, but two hodoscopes at TREAT have undergone major changes that practically constitute new systems. Several hodoscope systems have been designed but not built for other reactors.

In existing experiments, three-dimensional capability has been overrated as a goal and unachievable in practice. For large-bundle experiments of the future, three-dimensional time-resolved capability might be sought if the facility could offer viewing through right-angle slots. Other improvements are anticipated so that the hodoscope might have adaquate sensitivity for larger test bundles.

Nearly two decades of experience with high-resolution material-motion diagnostic systems have demonstrated an essential role for cineradiographic instrumentation in nuclear-reactor-safety experiments. Witnout dynamic quantitative and dimensional measurements, in-pile experiment data could not be properly related by computer models to prototypical reactors, nor could the data-adjusted models be adequately validated for licensing purpcses. A broader function for hodoscope diagnostic information is now emerging in terms of time- and space-resolved determination of fuel-pin power coupling during transient reactor irradiations. 


\section{References}

[AL-82]R.W. Albrecht, R.M. Wu, and R.P. Omberg, "TREAT Fodoscope Interpretation," Trans. Intl. Topical Mtg. on LMFBR Safety, Lyon (to be published).

[AR-81] Y. Arata, "Basic Phenomena in High Energy Density Beam Welding and Cutting," Proc. of the First European Conf. on Cineradiography, SPIE-Intl. Soc. for opt. Eng., 312 (to be published).

[AV-79] R. Avery, C.E. Dickerman, D.H. Lennox, and D. Rose, "Fast Reactor Test Facilities in the U.S. Safety Program," Proc. of the Intl. Meeting on Fast Reactor Safety Technology, American Nuclear Society (1979).

[BA-80] C.W. Bates, Jr., "Concepts and Implementations in X-Ray Image Intensification," in Garrett and Bracher.

[BAR-76] H.H. Barrett, "A Survey of Coded Aperture Imaging," Trans. Conf. on Fuel and Clad Motion Diagnostics in LMFBR Safety Test Facilities, Sandia Laboratories report SAND76-5547 (1976).

[BE-61] L.A. Beach, A.G. Pieper, and M.P. Young, "Gamma-Ray Scanning Techniques for Fast Breeder Reactor Safety Studies, "Naval Research Laboratory rejort NRL-5725 (July 1961).

[BE-76a] G.J. Berzins and Ki S. Han, "Possible Application of Nonredundant Pinhole Arrays to Fuel Pin Imaging," Trans. Conf. on Fuel and Clad Motion Diagnostics in LMFBR Safety Test Facilities, Sandia Laboratories report SAND 76-5547 (1976).

[BE-76b] G.J. Berzins, K.S. Han, and W.H. Roach, "Preliminary Report on the Pinex at TREAT," Trans. Of the Second Technical Exchange Meeting on Fuel- and Clad-motion Diagnostics for LMFBR Safety Test Facilities, Argonne National Laboratory report ANL/RAS 76-34 (1976).

[BE-79] G.J. Berzins and A.H. Lumpkin, "PINEX-2: Pinhole-TV Imaging of Fuel Ejection from an Internally Venied Capsule," Proc. of the Intl. Mtg. on Fast Reactor Safety Technology, American Nuclear Society (1979).

[BEN-81] M. Bender, N. Alem, G. Nusholtz, and J.W. Melvin, "High-Speed Cineradiographic System for Biomechanics Impact Research," Prns. of the First European Conf. on Cineradiography, SPIE-Intl. Soc. for Opt. Eng., 312 (to be published).

[BI-76] F. Biggs and J.H. Renken, "Analysis of In-Core Fuel Motion Detection," Trans. Conf. on Fuel and Clad Motion Diagnostics in LMFBR Safety Test Facilities, Sandia Laboratories report SAND76-5547 (1976).

[BL-76a] H. Bluhm, K. Bohnel, M. Kuchle, H. Werle, R. Agaisse, J.M. Aujollet, and J. Dadillon, "Design Characteristics of the CABRI Neutron Hodoscope," Trans. Conf. on Fuel and Clad Motion Diagnostics in LMFBR Safety Test Facilities, Sandia Laboratories report SAND76-5547 (1976). 
[BL-76b] H. Bluhm, K. Bohnel, M. Kuchle, R. Agaisse, J.M. Aujollet, and J. Dadilion, "The CABRI Hodoscope," Trans. of the Second Technical Exchange Meeting on Fuel- and Clad-motion Diagnostics for LMFBR Safety Test Facilities, Argonne National Laboratory report ANL/RAS 76-34 (1976).

[BL-81] D.M. Blake, "The Flash X-Ray of Shot Exit from Gun Muzzles," Proc. of the First European Conf. on Cineradiography, SPIE-Intl. Soc. for 0pt. Eng., 312 (to be published).

[B0-79] K. Bohnel and H. Bluhm, "First Results of the CABRI Neutron Hodoscope," p. 2261, Proc. of the Intl. Meeting on Fast Reactor Safety Technology, American Nuclear Society (1979).

[BOS-81] R.H. Bossi and C.T. Oien, "Real-Time Radiography," Lawrence Livermore National Laboratory report UCRL-53091 (1981).

[BOS-82] R.H. Bossi, A.H. Robinson, and J.P. Barton, "High Frame-rate Neutron Radiography of Dynamic Events," Trans. World Conf. on Neut. Rad. (1982).

[BR-81] R.j. Bracher, "High Speed Cine Radiography of Projectiles, "Proc. of the First European Conf. on Cineradiography, SPIE-Intl. Soc. for Opt. Eng., 312 (to be published).

[BR-82] W.R. Brody, "Digital Subtraction Angiography," IEEE Trans. Nucl. Sci. NS-29(3), 1176 (1982).

[CA-81] C. Cavailler, N. Fleurot, and R. Sauneuf, "Cinematographie Ultra Rapide Dans le Domaine Spectral X Destinee Aux Experiences D'Interaction Laser-matiere," Proc. of the First European Conf. on Cineradiography, SPIEIntl. Soc. for Opt. Eng., 312 (to be published).

[CH-76] L.M. Choate, J.A. Halbleib Sr., and L.D. Posey, "Sandia Flash XRadiography for Fuel Motion Studies," Trans. of the Second Technical Exchange Meeting on Fuel- and Clad-motion Diagnostics for LMFBR Safety Test Facilities, Argonne National Laboratory report ANL/RAS 76-34 (1976).

[CH-80] V. Chalmeton, "Microchannel X-Ray Image Intensifiers," in Garrett and Bracher.

[CHI-80] J. Chikawa, "Video Display of X-Ray Topographic Images for InProcess Inspection of Semiconductor Devices," in Garrett and Bracher.

[COX-76a] S.A. Cox, K.A. Beyerlein, and F.M. Levert, "Development of an InCore Directional Fuel Motion Monitor," Trans. Conf. on Fuel and Clad Motion Diagnostics in LMFBR Safety Test Facilities, Sandia Laboratories report SAND 76-5547 (1976).

[Cox-76b] S.A. Cox, R.A. Beyerlein, and F.E. LeVert, "Development of In-core Directional Neutron and Gamma Ray Sensors for Fuel Motion Detection, "Trans. of the Second Technical Exchange Meeting on Fuel- and Clad-motion Diagnostics for LMFBR Safety Test Facilities (1977). 
[DA-30] J.B. Davidson and A.L. Case, "Neutron Diffraction Tomography: A Unique 3D Inspection Technique for Crystals Using an Intensifier TV System," in Garrett and Bracher.

[DA-82] M.V. Davis, H. Berger, and F. Patricelli, "Real-Time Neutron Radiography at the Georgia Tech. Research Reactor," Trans. Am. Nucl. Soc. $\underline{41}$, 241 (1982).

[DEV-66] A. De Volpi and C.H. Freese, "Fast Neutron Transient Detection Hodoscope for Reactor Fuel Meltdown Studies," IEEE Trans. Nucl. Sci. NS-13 (1), 623 (1966).

[DEV-75] A. De Volpi, R.J. Pecina, R.T. Daly, D.J. Travis, R.R. Stewart, and E.A. Rhodes, "Fast-Neutron Hodoscope at TREAT: Development and Operation," Nucl. Techn. 27: 449 (1975).

[DEV-76a] A. De Volpi, R.R. Stewart, J.P. Regis, G.S. Stanford, and E.A. Rhodes, "Fast-Neutron Hodoscope at TREAT: Data Processing, Analys is, and Results," Nucl. Techn. 30: 398 (1976).

[DEV-76b] A. De Volpi, "High-resolution Fast-neutron and Gamma Digital Radiography," IEEE Trans. Nucl. Sci. NS-23 (1), 350 (1976).

[DEV-76c] A. De Volpi, C.L. Fink, and R.R. Stewart, "Monitoring Clad Blockages," Trans. Conf. on Fuel and Clad Motion Diagnostics in LMFBR Safety Test Facilities, Sandia Laboratories report SAND76-5547 (1976).

[DEV-76d] A. De Volpi, "Sodium Void Detection," Trans. of the Second Technical Exchange Meeting on Fuel- and Clad-motion Diagnostics for LMFBR Safety Test Facilities, Argonne National Laboratory report ANL/RAS 76-34 (1976).

[DEV-77a] A. De Volpi, "Fuel and Cladding Motion Surveillance," Proceedings of the Intl. Mtg. on Fast Reactor Safety and Related Physics, USERDA CONF761001 II: 846 (1977).

[DEV-78] A. De Volpi, "High Resolution Radiography by Means of a Hodoscope," U.S. Patent 4092542 (30 May 1978).

[DEV-79] A. De Volpi, P. Bertoncini, C. Fink, A. Klickman, L.W. Person, ᄃ. Rhodes, G.S. Stanford, and R. May, "Current and Advanced Methods for Determining Quantitative Fuel Dispersion Using Hodoscope Data," p. 2279 , Proc. of the Intl. Meeting on Fast Reactor Safety Technology, American Nuclear Society (1979).

[DEV-81a] P. De Volpi, "Hodoscope Cineradiography of Nuclear Fuel Destruction Experiments," Proc. of the First European Conf. on Cineradiography, SPIE-Int1. Soc. for 0pt. Eng., 312 (to be published).

[DEV-81b] A. De Volpi, G.E. Marsh, and E. Rhodes, private communication (September 1981). 
[DEV-82a] A. De Volpi, C.L. Fink, G.E. Marsh, E.A. Rhodes, and G.S. Stanford, "Fast-neutron Hodoscope at TREAT: Methods for Quantitative Determination of Fuel Dispersal," Nucl. Techn. (1982).

[DEV-82b] A. De Volpi, "Time-resolved Neutron Radiography of Reactor Fuel," Trans. World Conf. on Neut. Rad. (to be published).

[DEV-82c] A. De Volpi and E.A. Rhodes, "Reactor In-situ Neutron Radiography," Trans. World Conf. on Neut. Rad. (to be published).

[DEV-82d] A. De Volpi, "In-situ Material-motion Diagnostics and Fuel Radiography in Experimental Reactors," Trans. Conf. on Fast Thermal, and Fusion Reactor Experiments, Salt Lake City (to be published).

[DEV-82e] A. De Volpi, "Unjustified Flux Anomaly at TREAT," submitted to Nucl. Sci. \& Eng. (1982).

[DEV-82f] A. De Volpi and E.A. Rhodes, "Neutron and Gamma-ray Tomographic Imaging of LMFBR SAREF-program Safety-test Fuel Assemblies, "Materials Evaluation (to be published).

[DI-61] C.E. Dickerman, E.S. Sowa, D. Okrent, J. Monaweck, and L.B. Miller, "Studies of Fast. Reactor Fuel Element Behavior Under Transient Heating to Failure," Argonne National Laboratory report ANL-6334 (1961).

[DI-70] C.E. Dickerman, "Studies of Fast Reactor Core Behaviour Under Accident Conditions," Nucl. Safety 11, 195 (1970).

[DI-81] R.D. Dick, "PHERMEX Applications to Study High-Pressure Flow and Detonation Waves," Proc. of the First European Conf. on Cineradiography, SPIE-Intl. Soc. for Opt. Eng., 312 (to be published).

[DU-76] S.A. Dupree, "Accuracy of Fuel Motion Measurements Using In-Core Detectors," Trans. Conf. on Fuel and Clad Motion Diagnostics in LMFBR Safety Test Facilities, Sandia Laboratories report SAND76-5547 (1976).

[EB-81] D. Ebeling, F. Frungel, and H. Martinen, "Repetitive Sub-microsecond Light, $X$-ray and Spark Tracing Technics and Applications in Precision Analys is of Motions and Flows," Proc. of the First European Conf. on Cineradiography, SPIE-Intl. Soc. for Opt. Eng., 312 (to be published).

[ED-76] L.R. Edwards, "Fuel Motion Measurements with In-Core Detectors," Trans. Conf. on Fuel and Clad Motion Diagnostics in LMFBR Safety Test Facilities, Sandia Laboratories report SAND76-5547 (1976).

[EV-79a] A.E. Evans, Jr. and J.D. Orndoff, "Progress in Fuel-Motion Diagnostics: Instrumentation Evaluation at PARKA," Proc. of the Intl. Meeting on Fast Reactor Safety Technology, American Nuclear Society (1979).

[EV-79b] "Evaluation of LMFBR Fuel-motion Diagnostics Instrumentation with PARKA," IEEE Trans. Nucl. Sci., NS-16(1), 815 (1979). 
[EV-80] A.E. Evans and J. Orndoff, "Evaluation of Techniques for Dynamic Measurement of Fuel Motion in Liquid-Metal-Cooled Fast-Breeder Reactor Safety Experiments," U.S. NRC report NUREG/CR-1398 and U.S. DOE report LA-8301 (1980).

[FI-76] C.L. Fink, A. De Volpi, and G. Stanford, "Auvances in Clad Blockage Detection," Trans. of the Second Technical Exchange Meeting on Fuel- and Clad-motion Diagnostics for LMFBR Safety Test Facilities, Argonne National Laboratory report ANL-6457 (1977).

[FI-77] C.L. Fink, A. De Volpi, and C.A. Ford, "Status Report on CladBlockage and Sodium Void Detection," Specialists" Meeting on Fuel and Cladding Motion Diagnostics, Los Alamos, NM (Dec. 1977), (unpublished).

[Fl-79] C.L. Fink, A. De Volpi, and E.A. Rhodes, "Hodoscope Performance Tests on a 91-pin Fuel Bundle at PARKA," IEEE Trans. Nucl. Sci., NS-26(1), 827 (1979).

[Fl-80] C.L. Fink, J.J. Eichholz, D.R. Burrows, and A. De Volpi, "Protonrecoil Proportional Counter Tests at TREAT," IEEE Trans. on Nucl. Sci., NS-27 , 833 (1980).

[FI-82a] C.L. Fink, "Optimization of a Hornyak-button Detector for Fastneutron Detection," IEEE Trans. on Nucl. Sci., NS-29, 718 (1982).

[FI-82b] C.L. Fink, R.E. Boyar, J.J. Eichholz, and A. De Volpi, "Improvements in TREAT Hodoscope Fuel-motion Capabilities," Trans. Conf. on Fast, Thermal, and Fusion Reactor Expts., Salt Lake City, Utah (to be published).

[GAR-80] D.A. Garrett and D.A. Bracher (Eds.), Real-time Radiologic Imaging: Medical and Industrial Applications, Am. Soc. for Testing and Materials, Phila., PA (1980).

[G0-62] G.H. Golden, C.E. Dickerman, and L.E. Robinson, "Facility for Photographing In-Pile Meltdown Experiments in TREAT, "Argonne National Laboratory report ANL-6457 (1962).

[HA-76] J.A. Halbleib, "X-Ray Cinematography of Fuel Pin Motion," Trans. Conf. on Fuel and Clad Motion Diagnostics in LMFBR Safety Test Facilities, Sandia Laboratories report SAND76-5547 (1976).

[HA-80] R. Halmshaw, "Fundamentals of Radiographic Imaging," in Garrett and Bracher.

[HA-80] W. Hartmann, "A Real-Time X-Ray Topography System," in Garrett and Bracher.

[HAN-76a] K. S. Han and G.J. Berzins, "Two-Dimensional Nonredundant Arrays for Coded Aperture Imaging," Trans. Conf. on Fuel and Clad Motion Diagnostics in LMFBR Safety Test Facilities, Sandia Laboratories report SAND76-5547 (1976). 
[HAN-76b] K.S. Han, G.J. Berzins, and W.H. Roach, "Psudo Real-Time Imaging Systems with Nonredundant Pinhole Arrays," Trans. of the Second Technical Exchange Meeting on Fuel- and Clad-motion Diagnostics for LMFBR Safety Test Facilities, Argonne National Laboratory report ANL/RAS 76-34 (1976).

[HAN-76c] K.S. Han, G.J. Berzins, and W.H. Roach, "A Proposed Coded Aperture Imaging System for LMFBR Safety Experiments," Los Alamos Scientific Laboratory report LA-6208-P (1976).

[HAR-81] M.L. Harris, "The Dynamic Spatial Reconstructor," Proc. of the First European Conf. on Cineradiography, SPIE-Intl. Soc. for Opt. Eng., 312 (to be published).

[HAU-81] A. Hauducoeur, D. Fischer, and R. Guix, "Visualisation Radiographique de Phenomenes Ultra-rapides Application au Comportement des Materiaux Soumis aux Effets D'un Explosif," Proc. of the First European Conf. on Cineradiography, SPIE-Intl. Soc. for 0pt. Eng., 312 (to be published).

[HE-82] H.H. Helmick, K. Baumung, and K. Boehnel, "Recent Activities of the CABRI Hodoscope," Trans. Intl. Topical Mtg. on LMFBR Safety, Lyon (to be published).

[JA-80] J.E. Jacobs, "X-Ray-Sensitive Television Camera Tubes," in Garrett and Brächer.

[J0-82] J. John, W. Dance, and W.L. Whittemore, "Performance of Isotope and Accelerator Systems for Mobile, Real-time, and Cold Neutron Applications," Trans. World Conf. Neut. Rad. (to be published).

[KE-76a] J.G. Kelly, D.A. McArthur, and H.H. Barrett, "Coded Aperture XRadiography," Trans. Conf. on Fuel and Clad Motion Diagnostics in LMFBR Safety Test Facilities, Sandia Laboratories report SAND76-5547 (1976).

[KE-76b] J.G. Kelly, "Coded Source X-Radiography," Transactions of the Second Technical Exchange Meeting on Fuel- and Clad-motion Diagnostics for LMFBR Safety Test Facilities, Argonne National Laboratory report ANL/RAS 76-34 (1976).

[KE-76c] J.G. Kelly and K.G. Stalker, "Sandia ACPR Upgrade Fuel Motion Detection System," Trans. of the Second Technical Exchange Meeting on Fueland Clad-motion Diagnostics for LMFBR Safety Test Facilities (1977).

[KE-79] J.G. Kelly, K.T. Stalker, D.A. MCArthur, K.W. Chu, and J.E. Powell, "Theory and Application of the Coded Aperture Fuel Motion Detection System," p. 2302, Proc. of the Intl. Meeting on Fast Reactor Safety Technology, American Nuclear Society (1979).

[KE-82] J.G. Kelly and K.T. Stalker, "Results of Recent Tests of the Improved ACRR Coded Aperture Imaging System," Trans. Intl. Topical Mtg. on LMFBR Safety, Lyon (to be published).

[KI-80] J.D. Kingsley, "X-Ray Phosphors and Screens," in Garrett and Bracher. 
[KR-80] R.A. Kruger, S.J. Riederer, T.L. Houk, C.A. Mistretta, C.G. Shaw, and J.C. Lancaster, "Real-Time Computerized Fluoroscopy and Radiography--A Progress Report," in Garrett and Bracher.

[KU-80] W. Kuhl, "Detection of X-Rays for Real-Time Imaging," in Garrett and Bracher.

[KUR-80] M. Kuriyama, W.J. Boettinger, and H.E. Burdette, "Basic Limits in Real-Time Industrial Radiographic Systems," in Garrett and Bracher.

[LA-80] A.M. Landraud and J.J. Clair, "Real-Time Optical Processing: Automatized uptical Plotter and Interferometric Device for 3D-Imaging," in Garrett and Bracher.

[LU-79] A.H. Lumpkin, G.J. Berzins, R.A. Cosimi, T.E. O'Hare, and J.R. Davidson, "Developments in the LASL Fuel Pin Imaging System: PINEX-3A," p. 2243, Proceedings of the International Meeting on Fast Reactor Safety Technology, American Nuclear Society (1979).

[LU-80] A.H. Lumpkin, private communications (1 May 1980); (3 September 1980); (3 December 1980).

[LU-81] A.H. Lumpkin, G.J. Berzins, J.R. Davidson, E.L. Miller, and W.A. 0lsen, private communication (29 May 1981).

[MA-80] G.E. Marsh, J.P. Regis, and R.R. Stewart, private communication (24 April 1980); (3 December 1980).

[MAT-81] A. Mattsson, "Flash X-ray Systems," Proc. of the First European Conf. on Cineradiography, SPIE-Intl. Soc. for 0pt. Eng., 312 (to be published).

[MAR-81] J. Marilleau, "Prospective en Cineradiographie et Cinetomographie a $L$ 'Aide des Accelerateurs de Particules, "Proc. of the First European Conf. on Cineradiography, SPIE-Intl. Soc. for Opt. Eny., 312 (to be published).

[MC-76] D.A. McArthur and J.G. Kelly, "A Coded Aperture Material Motion Detection System for the ACPR," Trans. Conf. on Fuel and Clad Motion Diagnostics in LMFBR Safety Test Facilities, Sandia Laboratories report SAND76-5547 (1976).

[MC-82] M.L. McConne11, D.J. Forrest, E.L. Chopp, and P.P. Dunphy, "A Coded Aperture Gamma Ray Telescope," IEEE Trans. Nucl. Sci., NS-29(1), 155 (1982).

[ME-80] P. Mengers, "Real-Time Digital Image Processing for Radiological Imaging," in Garrett and Bracher.

[ME-82] J.C. Mel is, J.M. Frizonnet, J.M. Berniolles, K. Lassmann, D. Struwe, D. Freund, M.H. Wood, and D. Moxon, "Analys is of the CABRI In-pile Experiments," Trans. Intl. Topical Mtg. on LMFBR Safety, Lyon (to be published). 
[MI-77] G.N. Minerbo and J.G. Sanderson, "Reconstruction of a Source from a Few (2 or 3) Projections, "Los Alamos Scientific Laboratory report LA-6747-MS (1977).

[M0-70] N.F. Moody, W. Paul, and M.L.G. Joy, "A Survey of Medical Gamma-ray Cameras" Proc. IEEE 58(2), 217 (1970).

[NO-80] B.W. Noel and A.J. Yates, "FPS-Vidicon Television Cameras for Ultrafast-Scan Data Acquisition," Los Alamos Scientific Laboratory report LA8236 (1980).

[PA-81] F. Patricelli and L. Corso, "Real-time Neutron Radiography," Trans. World Conf. on Neut. Rad. (1982).

[PE-78] L.W. Person, A.E. Klickman, and E. Rhodes, "Use of Hodoscopes to Obtain Tomographic Resolution of Fuel Motion," Trans. Am. Nucl. Soc. 28, 127 (1978).

[P0-76] L.D. Posey, "Source Requirements for Flash X-Radiography Diagnostics," Trans. Conf. on Fuel and Clad Motion Diagnostics in LMFBR Safety Test Facilities, Sandia Laboratories report SAND76-5547 (1976).

[P0-80] D. Polansky, "Radiation Sources and Detectors," in Garrett and Bracher.

[RH-76a] E.A. Rhodes: "Evaluation of Ex-core Imaging Apertures for STF," Transactions of the Second Technical Exchange Meeting on Fuel-and Cladmotion Diagnostics for LMFBR Safety Test Facilities, Argonne National Laboratory report ANL/RAS 76-34 (1976).

[RH-76b] E. Rhodes, A. De Volpi, C. Fink, G. Stanford, and R. Stewart, "Hodoscope In-Situ Radiography," Transactions of the Second Technical Exchange Meeting on Fuel- and Clad-Motion Diagnostics for LMFBR Safety Test Facilities, Argonne National Laboratory report ANL/RAS 76-34 (1976).

[RH-78] E. Rhodes and J.P. Regis, private communication (April 1978); also E. Rhodes, private communication (25 September 1978).

[RH-79] E.A. Rhodes, A. De Volpi, C. Fink, G. Stanford, R. Pecina, D. Travis, and R. Kash, "TREAT Fast-neutron Hodoscope: Improvements in Time and Mass Resolution of Fuel Motion," IEEE Trans. on Nucl. Sci., NS-26, 809 (1979).

[RH-82a] E.A. Rhodes and A. De Volpi, "Image Restoration to Increase Resolution of the Fast-neutron Hodoscope," Trans. World Conf. on Neut. Rad. (to be published).

[RH-82b] E.A. Rhodes and A. De Volpi, "Image Processing and Resolution Restoration of Fast-neutron Hodoscope Data," Trans. IEEE Comp. Soc. (t.o be published).

[RH-82C] E.A. Rhodes, J.P. Barton, C.F. Barton, G.A. Schlapper, R.M. Brugger, A. De Volpi, C.E. Dickerman, and D. Rose, "Ex-core Neutron Radiography of Large Fuel Bundles," Trans. World Conf. on Neut. Rad. (to be published). 
[RI-80] E.L. Ritman, R.A. Robb, L.D. Harris, J.H. Kinsey, B.K. Gilbert, K.T. Smith, and E.H. Wood, "Cylindrical-Scanning Transaxial Roentgen Tomography of High Temporal Resolution," in Garrett and Bracher.

[RU-80] R.G. Kudolph and E.B. Henry, Jr., "Real-Time Radiographic Imaging for Submerged-Arc Welded Pipe," in Garrett and Bracher.

[SA-81] M. Saudinos and C. Charpak, "Applications Industrielles et Medicales de la Radiographie 3 D," Proc. of the First European Conf. on Cineradiography. SPIE-Intl. Soc. for Opt. Eng., 312 (to be published).

[SA-82] Advanced Reactor Safety Research Quarterly Report, Sandia National Laboratories report SAND81-1529 (NUREG/CR-2238) (1982).

[SC-80] C. Scheid, "Performance Measurement of Fluoroscopic Systems," in Garrett and Bracher.

[SC-81] R.A. Schrack, J.W. Behrens, R.G. Johnson, and C.D. Bowman, "Resonance Neutron Radiography," Trans. World Conf. on Neut. Rad. (to be published).

[SI-80] M.P. Siedband, "Beam Filtration in Diagnostic Radiology," in Garrett and Bracher.

[SI-81] R. Simms, G.E. Marsh, A.B. Rothman, and G.S. Stanford, "Loss-of-Flow Transient Reactor Test Facility Tests L6 and L7 with Irradiated Liquid-Metal Fast Breeder Reactor Type Fuel," Nucl. Techn. 52:331 (1981).

[SK-80] A.F. Sklensky and R.A. Buchanan, "Sensitivity of High-Energy RealTime Radiography with Digital Integration," in Garrett and Bracher.

[SM-82] D.E. Smith, F.J. Martin, A. Padilla, Jr., and A.E. Waltar, "Internal Fuel Motion as an Inherent Safety Shutdown Mechanism During Hypothetical LMFBR Accidents," Trans. Intl. Topical Mtg. on LMFBR Safety, Lyon (to be published).

[SP-82] B. Spencer and D.H. Cho, "Phenomenological Studies of HCDA Issues," Trans. Intl. Topical Mtg. on LMFBR Safety, Lyon (to be published).

[ST-76a] W.E. Stein, V. Starkovich, and J.D. Orndoff, "K-ray Monitoring of Fuel Motion," Transactions of the Second Technical Exchange Meeting on Fueland Clad-motion Diagnostics for LMFBR Safety Test Facilities, Argonne National Laboratory report ANL/RAS 76-34 (1976).

[ST-76b] W.E. Stein, "Flash X-Ray Cinematography," Trans. Conf. on Fuel and Clad Motion Diagnostics in LMFBR Safety Test Facilities, Sandia Laboratories report SAND76-5547 (1976).

[ST-79] K.T. Stalker, L.M. Choate, and L.D. Posey, "The Potential of High Speed X-Ray Cinematography as a Fuel Motion Diagnostic for Safety Test Facilities," p. 2270, Proc. of the Intl. Meeting on Fast Reactor Safety Technology, American Nuclear Society (1979). 
[ST-80] K.T. Stalker and J.G. Kelly, "Coded aperture imaging system for nuclear fuel motion detection, "Proc. Soc. Photo-0pt. Instrum. Eng. 231, 12 (1980).

[ST-80] P.A.E. Stewart "Cold Neutron Imaging for Gas Turbine Inspection," in Garrett and Bracher.

[ST-81] P.A.E. Stewart and A.I. Lewcock, "Aero Engine Applications of Cold Neutron Imaging at Rolls Royce," Trans. World Conf. on Neut. Rad. (to be published).

[STR-81] M.G. Strauss, G.H. Lander and R. Brenner, "Resonance NR Using a Pulsed Neutron Source and a Two-dimensional Scintillation Detector," Trans. World Conf. on Neut. Rad. (to be published).

[TW-82] R.E. Twogood and F.G. Sommer, "Digital Image Processing," IEEE Trans. Nucl. Sci., NS-29(3), 1076 (1982).

[WA-81] A.E. Waltar and A.B. Reynolds, Fast Breeder Reactors, Pergamon Press, NY (1981).

[WI-81] H. Will, P. Menzenhauer, and W. Peppler, "Investigation of Material Movement within Steel Testsections by Means of High Speed X-ray Photography," Proc. of the First European Conf. on Cineradiography, SPIE-Intl. Soc. for Opt. Eng., 312 (to be published).

[WR-76] S.A. :Uright and S.A. Dupree, "In-core Fuel Motion Detection for Large Scale Tests," Trans. of the Second Technical Exchange Meeting on Fuel- and Clad-motion Diagnostics for LMFBR Safety Test Facilities (1977).

[WR-79] S.A. Wright, P.J. McDaniel, J.E. Powell, and W.H. Scott, Jr., "The SPR-III 7-Pin In-Core Fuel Motion Detection Feasibility Experiments," Proc. of the Intl. Melting on Fast Reactor Safety Technology, American Nuclear Society (1979).

[ZI-80] M.C. Ziskin and C.M. Philips, "Computerized Fluorodensitometry Using a Solid-State Matrix," in Garrett and Bracher. 


\section{Distribution for ANL-82-72}

Internal:
E. S. Beckjord
G. S. Stanford
C. E. Till
R. E. Boyar
R. Avery
D. R. Burrows
D. W. Cissel
L. J. Harrison
D. R. Ferguson
D. M. Ray
B. R. T. Frost
E. W. Walbridge
L. G. LeSage
S. S. Borys
R. J. Teunis
R. E. Herbert
A. DeVolpi (20)
ANL Patent Dept.
J. P. Regis
C. L. Fink
ANL Contract File
T. H. Braid
ANL Libraries (2)
E. A. Rhodes
TIS Files (6)

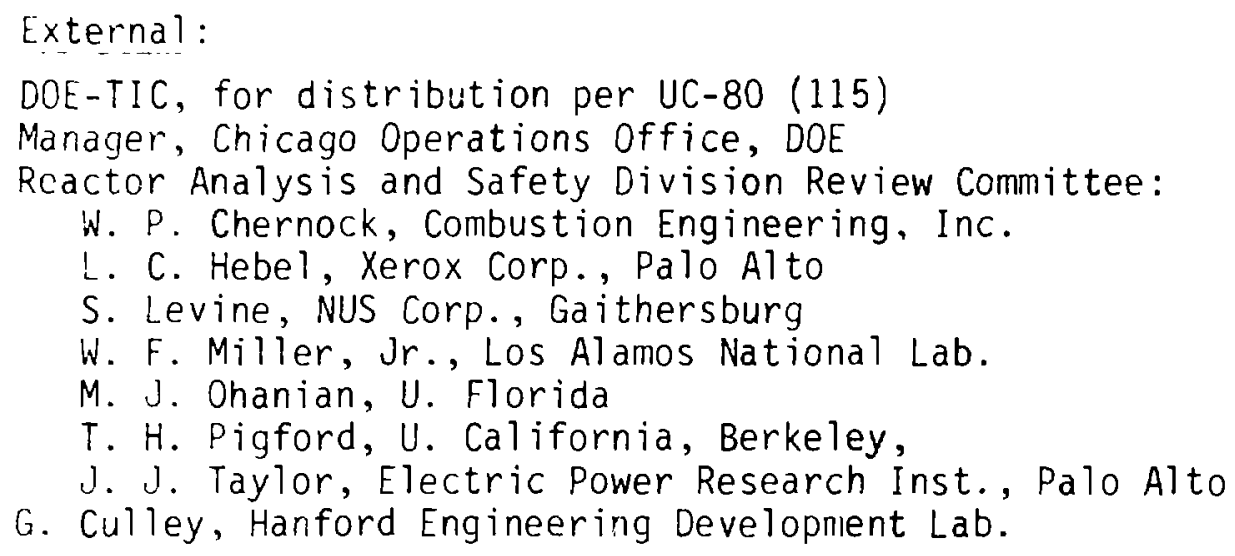

